\title{
Long-term magnetic activity in close binary systems ${ }^{\star}$
}

\section{Patterns of color variations $\star \star, \star \star \star$}

\begin{abstract}
S. Messina
INAF - Catania Astrophysical Observatory, via S. Sofia 78, 95123 Catania, Italy

e-mail: sergio.messina@oact.inaf.it

Received 26 October 2007 / Accepted 17 December 2007

ABSTRACT

Aims. This is the first of a series of papers in which we present the results of a long-term photometric monitoring project carried out at Catania Astrophysical Observatory aimed at studying magnetic activity in late-type components of close binary systems, its dependence on global stellar parameters, and its evolution on different time scales from days to years. In this first paper, we present the complete observation dataset and new results of an investigation into the origin of brightness and color variations observed in the well-known magnetically active close binary stars: AR Psc, VY Ari, UX Ari, V711 Tau, EI Eri, V1149 Ori, DH Leo, HU Vir, RS CVn, V775 Her, AR Lac, SZ Psc, II Peg and BY Dra

Methods. About 38000 high-precision photoelectric nightly observations in the $U, B$ and $V$ filters are analysed. Correlation and regression analyses of the $V$ magnitude vs. $U-B$ and $B-V$ color variations are carried out and a comparison with model variations for a grid of active region temperature and filling factor values is also performed.

Results. We find the existence of two different patterns of color variation. Eight stars in our sample: BY Dra, VY Ari, V775 Her, II Peg, V1149 Ori, HU Vir, EI Eri and DH Leo become redder when they become fainter, as is expected from the presence of active regions consisting of cool spots. The other six stars show the opposite behaviour, i.e. they become bluer when they become fainter. For V711 Tau this behaviour could be explained by the increased relative $U$ - and $B$-flux contribution by the earlier-type component of the binary system when the cooler component becomes fainter. On the other hand, for AR Psc, UX Ari, RS CVn, SZ Psc and AR Lac the existence of hot photospheric faculae must be invoked. We also found that in single-lined and double-lined binary stars in which the fainter component is inactive or much less active the $V$ magnitude is correlated to $B-V$ and $U-B$ color variations in more than $60 \%$ of observation seasons. The correlation is found in less than $40 \%$ of observation seasons when the fainter component has a non-negligible level of activity and/or hot faculae are present but they are either spatially or temporally uncorrelated to spots.
\end{abstract}

Key words. stars: activity - stars: binaries: close - stars: late-type - stars: magnetic fields - stars: starspots - techniques: photometric

\section{Introduction}

Studies of stellar magnetic activity and variability at Catania Astrophysical Observatory (OAC) date back to the late Sixties, when pioneering research was undertaken to explore the nature of stellar spots (Rodonò 1965) and stellar flares (Cristaldi \& Rodonò 1968; Cristaldi et al. 1968).

The existence of stellar spots, first proposed by Kron (1950) as a cause of the variability observed in a few late-type stars, was still debated at that time. The research at OAC significantly contributed to understand their nature and yielded relevant results such as the discovery of the characteristic outside-ofeclipse photometric or distortion wave on the light curve of the proto-type RS CVn system (Chisari \& Lacona 1965; Catalano \& Rodonò 1967) playing an important role in the identification of the new class of binaries named after $R S C V n$ (Oliver 1974; Hall 1976). Also stellar flare studies at OAC, carried out at the same time within the coordination of the Working Group on

* I dedicate this paper to the memory of the P.I. of this project, Prof. Marcello Rodonò, who suddenly passed away on October 23, 2005. To him my sincere estimation and deepest gratitude.

$\star \star$ Based on observations collected at INAF-Catania Astrophysical Observatory, Italy.

$\star \star \star$ Tables 4-7 are only available in electronic form at http://www . aanda.org
Flare Stars (Gershberg \& Shakhovskaya 2003), yielded relevant progress and resulted in the 71st IAU Colloquium on Activity in Red Dwarf Stars (Byrne \& Rodonò 1983).

Research on stellar spots, over many years, has progressively revealed that spots are non-stationary phenomena that after a few stellar rotations undergo, depending on the value of global stellar properties, sizeable changes in their dimension, number and surface distribution. Furthermore, if the star rotates differentially, spots at different latitudes produce different variability terms in the frequency domain and any initial spot distribution is significantly sheared after some rotations (Lanza et al. 1993, 1994). Since light curves undergo noticeable changes, active stars must be monitored as continuously as possible if we want to derive significant information on the behaviour of stellar activity. For this reason, the photometric monitoring at OAC became more and more systematic and was extended during the last three decades to a much larger sample of stars, either single or in close binary systems with a wide range of values of global properties (see, e.g., the series of papers by Cutispoto 1990, 1991, 1992, 1993, 1995). The photometric patrol, initially carried out with the $30-\mathrm{cm}$ and $91-\mathrm{cm}$ telescopes of OAC, was afterwards continued mainly with the use of two APTs, i.e. automatic photometric telescopes: the Phoenix-25 since 1988 (Rodonò \& Cutispoto 1992) and the Catania APT80/1, entirely dedicated to this project since 1992 (Rodonò et al. 2001b; Messina et al. 2004). Indeed, 
Table 1. Program stars: spectral type; rotation period; brightest $V$ magnitude $\left(V_{\min }\right)$, maximum light curve amplitude $\left(\Delta V_{\max }\right)$, mean colors, and total flux ratios $\left(L_{\mathrm{c}} / L_{\mathrm{h}}\right)$ in the $V, B$ and $U$ bands of the cool (c) to the hot (h) components.

\begin{tabular}{|c|c|c|c|c|c|c|c|c|c|c|c|}
\hline Program & HD & Name & Sp. type & Period & $V_{\min }$ & $\Delta V_{\max }$ & $\langle B-V\rangle$ & $\langle U-B\rangle$ & \multicolumn{3}{|c|}{$L_{\mathrm{c}} / L_{\mathrm{h}}$} \\
\hline star & number & & & (d) & (mag) & (mag) & (mag) & $(\mathrm{mag})$ & $V$ & $B$ & $U$ \\
\hline 1 & 8357 & AR Psc & K1 IV/V + G5/6 V & 12.38 & 7.243 & 0.186 & 0.836 & 0.383 & 5.6 & 4.8 & 3.5 \\
\hline 2 & 17433 & VY Ari & $\mathrm{K} 3 / 4 \mathrm{IV}+?$ & 16.3 & 6.690 & 0.421 & 0.979 & 0.649 & - & - & - \\
\hline 3 & 21242 & UX Ari & K0 IV + G5 V & 6.43971 & 6.362 & 0.273 & 0.852 & 0.438 & 11.2 & 9.5 & 6.9 \\
\hline 4 & 22468 & V711 Tau & $\mathrm{K} 1 \mathrm{IV}+\mathrm{G} 5 \mathrm{~V}$ & 2.83774 & 5.635 & 0.171 & 0.901 & 0.474 & 4.5 & 3.4 & 1.5 \\
\hline 5 & 26337 & EI Eri & G5 IV + G0 V & 1.94722 & 6.921 & 0.159 & 0.662 & 0.105 & 2.6 & 2.4 & 2.1 \\
\hline 6 & 37824 & V1149 Ori & $\mathrm{K} 2 / 3 \mathrm{III}+\mathrm{F} 8 \mathrm{~V}$ & 53.12 & 6.593 & 0.277 & 1.155 & 0.963 & 14.8 & 8.1 & 2.5 \\
\hline 7 & 86590 & DH Leo & K0 V + K7 V & 1.070354 & 7.811 & 0.079 & 0.898 & 0.481 & $6.5^{a}$ & $8.9^{a}$ & $20.8^{a}$ \\
\hline 8 & 106225 & HU Vir & $\mathrm{K} 1 \mathrm{IV}+?$ & 10.41 & 8.549 & 0.419 & 1.023 & 0.647 & - & - & - \\
\hline 9 & 114519 & RS CVn & $\mathrm{K} 0 \mathrm{IV}+\mathrm{F} 5 \mathrm{~V}$ & 4.797855 & 7.858 & 0.203 & 0.621 & 0.103 & 0.9 & 0.5 & 0.3 \\
\hline 10 & 175742 & V775 Her & $\mathrm{K} 0 \mathrm{~V}+\mathrm{K} 5 / \mathrm{M} 2 \mathrm{~V}$ & 2.879342 & 7.800 & 0.185 & 0.904 & 0.609 & $15.8^{a}$ & $22.2^{a}$ & $53.0^{a}$ \\
\hline 11 & 210334 & AR Lac & K2 IV + G2 IV & 1.98322195 & 6.030 & 0.160 & 0.768 & 0.725 & 1.4 & 1.2 & 0.9 \\
\hline 12 & 219113 & SZ Psc & K1 IV + F8 V/IV & 3.9657889 & 7.155 & 0.115 & 0.846 & 0.365 & 3.8 & 2.8 & 1.7 \\
\hline 13 & 224085 & II Peg & $\mathrm{K} 2 \mathrm{IV}+?$ & 6.720 & 7.283 & 0.671 & 1.031 & 0.761 & - & - & - \\
\hline 14 & 234677 & BY Dra & $\mathrm{M} 0 \mathrm{~V}+\mathrm{M} 0 \mathrm{~V}$ & 3.836 & 8.003 & 0.176 & 1.172 & 1.043 & 1.0 & 1.0 & 1.0 \\
\hline
\end{tabular}

${ }^{a}$ Total flux ratio of the hot to the cool component.

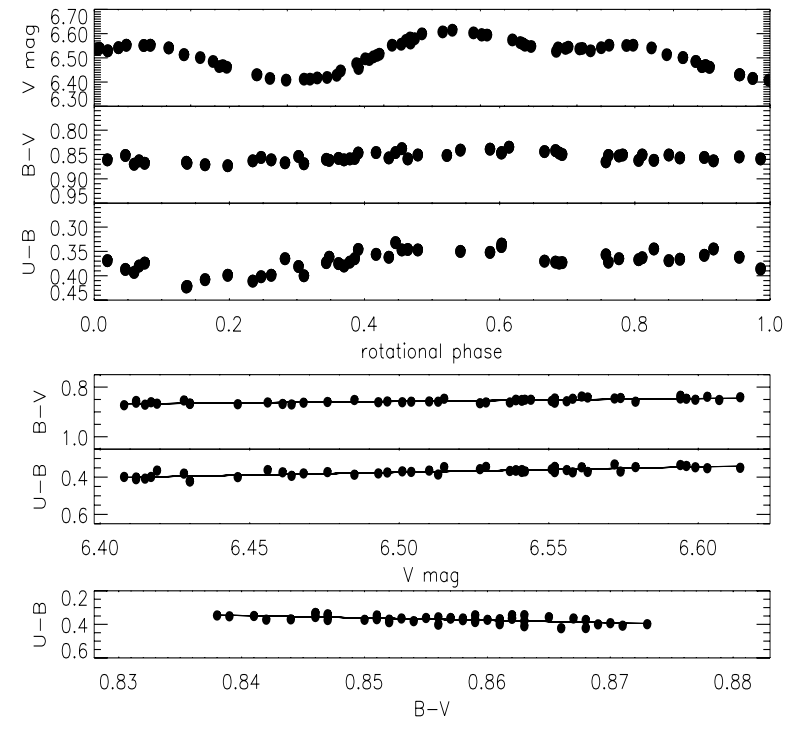

Fig. 1. Upper panels: $V$ magnitude, $B-V$ and $U-B$ colors of UX Ari vs. rotational phase for the mean epoch 1993.82. Lower panels: Color-magnitude ( $B-V$ and $U-B$ vs. $V$ ) and color-color $(U-B$ vs. $B-V$ ) variations along with a linear fit (solid line).

very high duty cycle and full automation have revealed the APTs to be best suited to obtain a homogeneous and systematic data base of high-quality multiband photometry of magnetically active stars (Rodonò 1992; Rodonò \& Cutispoto 1994a,b).

Starting from the Eighties, other institutions began similar long-term photometric monitoring projects with robotic telescopes. Among the most relevant projects, we just mention the Sun in Time undertaken by the Villanova University (see, e.g., Messina \& Guinan 2002, 2003). All these projects have made feasible a direct comparison between theoretical predictions and observational results, contributing significantly to increasing our knowledge of starspot properties, active region growth and decay (ARGD), activity cycles, surface differential rotation, active longitudes and flip-flop phenomena, orbital period variations and their dependence on stellar parameters.

In a series of papers we will present the final results on active close binary systems obtained by the long-term photometric monitoring project of OAC. For instance, the results of a similar project, but on single main-sequence stars, was presented in a previous series of papers (Messina \& Guinan 2002, 2003). In this first paper we investigate the origin of different patterns of color variations shown by active close binary systems. Starspot cycles and surface differential rotation will be the main subjects of subsequent papers.

The stellar sample and the photometric database are presented in Sects. 2 and 3. In Sect. 4 we investigate the correlation between color and magnitude variations on both short and long timescales. In Sect. 5 we describe a simple modelling approach to probe the nature of color variations. Discussion and conclusions are given in Sects. 6 and 7, respectively.

\section{The stellar sample}

From the whole stellar sample of about fifty stars monitored at OAC (see Rodonò et al. 2001b), we selected for the present analysis those stars for which we obtained the most extended data time series. The stellar sample analysed in this paper consists of 14 binary systems: six are SB1-type systems; eight are SB2-type systems, three of which are detached eclipsing binaries (see Table 1). In this section we give some information from the literature on their optical behaviour and the values of their physical parameters that will be used in the following to model the color variations. We indicate as the primary of the binary system the more massive and luminous component, not the one of earlier spectral type.

AR Psc (HD 8357) is an SB2 RS CVn-type variable consisting of a primary K1 subgiant and a secondary G5/6 dwarf (Cutispoto 1998). This system has an orbital period of $P_{\text {orb }}=$ $14.3023^{\mathrm{d}}$ (Fekel 1996), which is not synchronized with the rotational photometric period of $P_{\text {pho }}=12.38^{\mathrm{d}}$ (Cutispoto et al. 2001). The observed photometric variability is likely dominated by the more active and luminous subgiant component. However, the anticorrelation between $U-B$ and $V$ data found by Cutispoto (1995) may arise from the earlier-type component, as will be discussed. We adopt for the hot (h) and cool (c) components the following values from Strassmeier et al. (1993), Hongguam et al. (2006), and Cox (2000): $T_{\mathrm{h}}=5600 \mathrm{~K}, T_{\mathrm{c}}=4880 \mathrm{~K}, R_{\mathrm{h}}=$ $0.92 R_{\odot}, R_{\mathrm{c}}=2.7 R_{\odot}, \log g_{\mathrm{h}}=4.5, \log g_{\mathrm{c}}=3.0 \mathrm{~cm} \mathrm{~s}^{-2}, M_{\mathrm{h}} / M_{\mathrm{c}}=$ $0.82, i=37^{\circ}$. By using Eqs. (1) and (6) from Morris \& Naftilan (1993, and references therein), we computed that the variability 

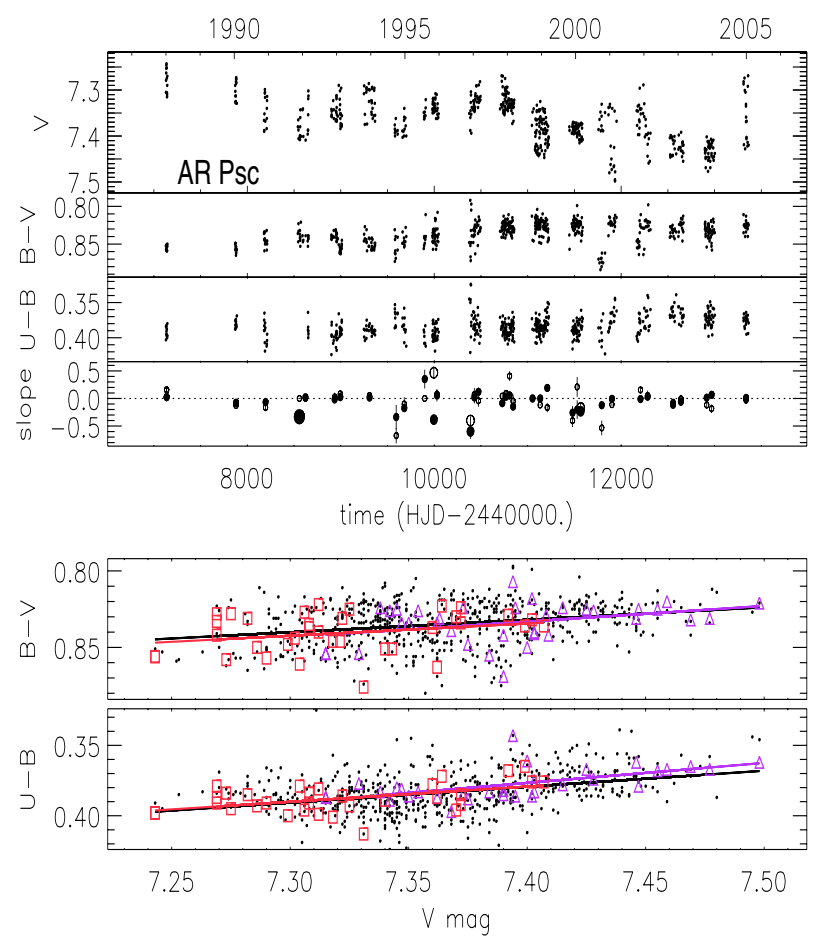
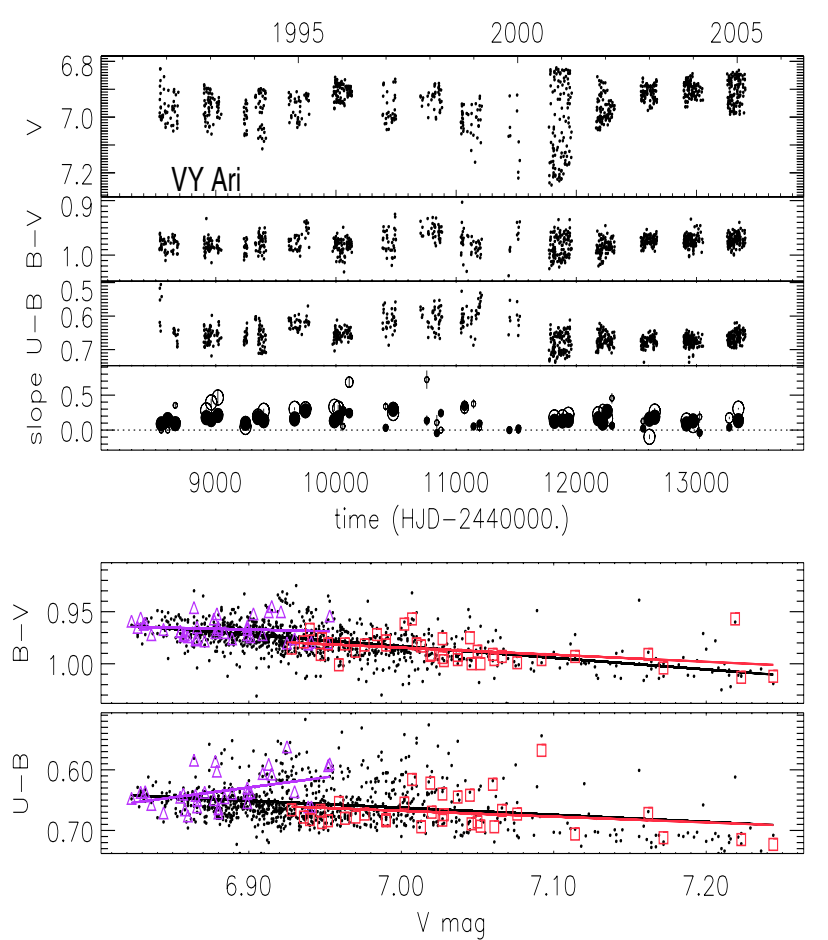

Fig. 2. Left: from top to bottom $V$-band magnitudes, $B-V$ and $U-B$ colors, and slopes of the linear fit to the $B-V$ vs. $V$ (filled circles) and $U-B$ vs. $V$ (open circles) relations vs. time for AR Psc. Larger symbol size indicates a larger significance level. Bottom: $B-V$ and $U-B$ colors vs. $V$ magnitudes (dots). Triangles and squares show the relation between bluest and brightest and between reddest and faintest light curve values, respectively. Solid lines are linear fits to the mentioned relations. Right: the same as in the left plot, but for VY Ari.

of AR Psc arising from proximity and reflection effects is about $\Delta V_{\text {ellip }}=6.0 \times 10^{-4} \mathrm{mag}$ and $\Delta V_{\text {ref }}=1.3 \times 10^{-4} \mathrm{mag}$.

VY Ari (HD 17433) is an SB1 RS CVn-type variable with a primary K3/4 subgiant. This system has an orbital period of $P_{\text {orb }}=16.42^{\mathrm{d}}$ (Strassmeier \& Bopp 1992), and a variable rotational photometric period of about $P_{\text {pho }} \sim 16.3^{\mathrm{d}}$ (Messina et al. 2004; Frasca et al. 2005). On the basis of the Li $6707.8 \AA$ abundance, a significant infrared excess and the non-synchronized orbital/rotational period, Bopp et al. (1989) suggest that this star could be a PMS system. We adopt the effective temperature from Frasca et al. (2005) and the surface gravity proper for its spectral class (Cox 2000): $T_{\mathrm{h}}=4900 \mathrm{~K}, \log g_{\mathrm{h}}=4.0 \mathrm{~cm} \mathrm{~s}^{-2}$. VY Ari will be treated in the analysis as a single star and proximity and reflection effects will be considered negligible.

UX Ari (HD 21242) is a triple system consisting of a SB2 RS CVn-type binary having a primary K0 subgiant and a secondary G5 dwarf (Cutispoto et al. 2001), and of a third faint late-type star (Duemmler \& Aarum 2001). This system has a rotational photometric period similar to the orbital period of $P_{\text {orb }}=6.43791^{\mathrm{d}}($ Carlos \& Popper 1971). UX Ari is known to have $B-V$ and $U-B$ color variations anticorrelated with the $V$-band flux variation, i.e. when the star becomes fainter it becomes bluer (Zeilik et al. 1982; Rodonò \& Cutispoto 1992). A flux contribution by the earlier-type component has been suspected as the cause of the color blueing (Wacker et al. 1986; Mohin \& Raveendran 1989). We adopt for the system's components the values of parameters from Aarum Ulvas \& Engvold (2003) and Strassmeier et al. (1993): $T_{\mathrm{h}}=5620 \mathrm{~K}, T_{\mathrm{c}}=4750 \mathrm{~K}$, $T_{3}=4400 \mathrm{~K}, R_{\mathrm{h}}=1.11 R_{\odot}, R_{\mathrm{c}}=5.78 R_{\odot}, R_{3}=0.70 R_{\odot}$, $M_{\mathrm{h}} / M_{\mathrm{c}}=0.80, i=60^{\circ}$, and the surface gravity proper for their spectral classes: $\log g_{\mathrm{h}}=4.5, \log g_{\mathrm{c}}=3.5, \log g_{3}=4.5 \mathrm{~cm} \mathrm{~s}^{-2}$. The flux from the third component has been properly scaled to take into account the parallax difference with respect to the
K0 IV + G5 V binary. We find that the variability of UX Ari arising from proximity and reflection effects is about $\Delta V_{\text {ellip }}=8.7 \times$ $10^{-3} \mathrm{mag}$ and $\Delta V_{\text {ref }}=4.3 \times 10^{-3} \mathrm{mag}$ (Morris \& Naftilan 1993).

V711 Tau (HD 22468) is an SB2 RS CVn-type variable consisting of a primary K1 subgiant and a secondary G5 dwarf (Fekel 1983). The RS CVn-type binary belongs to a visual binary, ADS 2644B (K3V) being its secondary component. The system has an orbital period of $P_{\text {orb }}=2.83774^{\mathrm{d}}($ Fekel 1983) which does not differ significantly from the photometric rotational period (Lanza et al. 2006). Although the observed photometric variability is dominated by the more active and luminous subgiant component, a flux contribution to the $B$ and $U$ bands by the earlier-type component has been suspected to be the cause of the color blueing (Aarum Ulvas \& Henry 2005). We adopt the parameter values for the hot and cool components from Lanza et al. (2006) and for the third component from Aarum Ulvas \& Engvold (2003): $T_{\mathrm{h}}=5500 \mathrm{~K}, T_{\mathrm{c}}=4750 \mathrm{~K}, T_{3}=4950 \mathrm{~K}$, $R_{\mathrm{h}}=1.1 R_{\odot}, R_{\mathrm{c}}=3.7 R_{\odot}, R_{3}=0.70 R_{\odot}, M_{\mathrm{h}} / M_{\mathrm{c}}=0.80, i=38^{\circ}$, and $\log g_{\mathrm{h}}=4.26, \log g_{\mathrm{c}}=3.3, \log g_{3}=4.5 \mathrm{~cm} \mathrm{~s}^{-2}$. We find that the variability of V711 Tau arising from proximity and reflection effects is about $\Delta V_{\text {ellip }}=0.040 \mathrm{mag}$ and $\Delta V_{\text {ref }}=3.3 \times 10^{-3} \mathrm{mag}$ (Morris \& Naftilan 1993). For this star Henry et al. (1995a) established that the proximity effect gives a much smaller contribution of about $\Delta V_{\text {ellip }}=0.017$.

EI Eri (HD 26337) is an SB1 RS CVn-type variable with a primary G5 subgiant and colors from UBVRI photometry consistent with a G5 IV + G0 V binary system (Cutispoto 1995). EI Eri has an orbital period of $P_{\text {orb }}=1.94722^{\mathrm{d}}$ (Fekel et al. 1987) and a variable rotational photometric period of about $P_{\text {pho }}=$ $1.94^{\mathrm{d}}$ (Strassmeier et al. 1997). If granted the spectral classification by Cutispoto (1995), the observed photometric variability may partly originate from the less-active main-sequence component, as will be discussed in the following. Evidence for an 

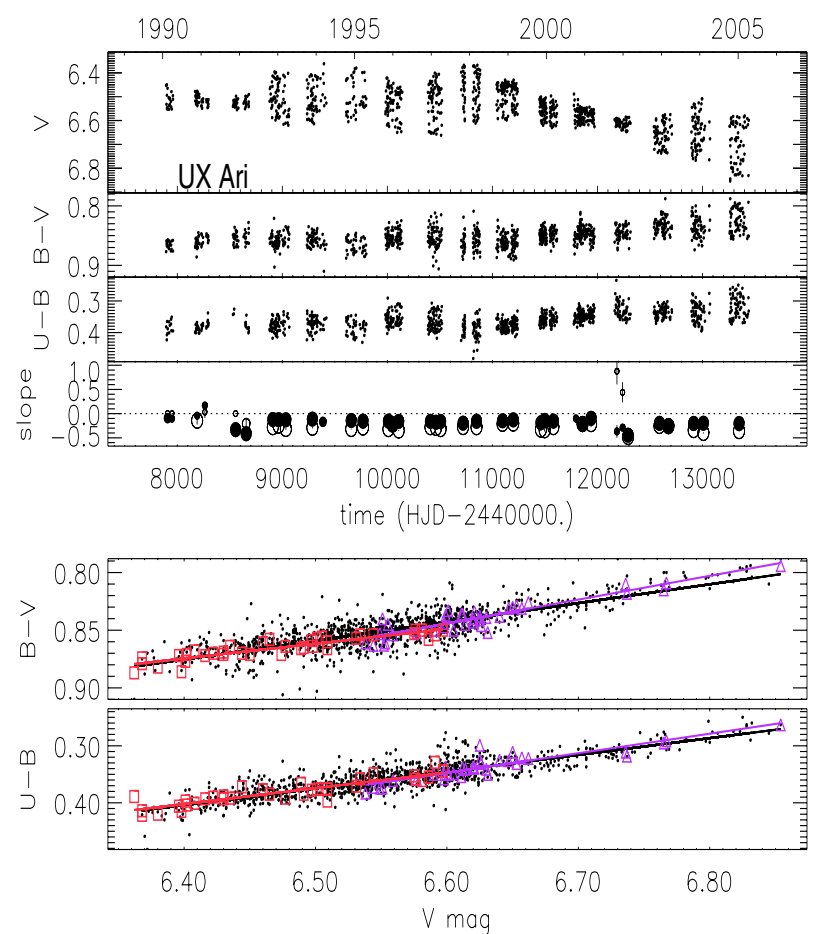

Fig. 3. As in Fig. 2, but for UX Ari and V711 Tau.
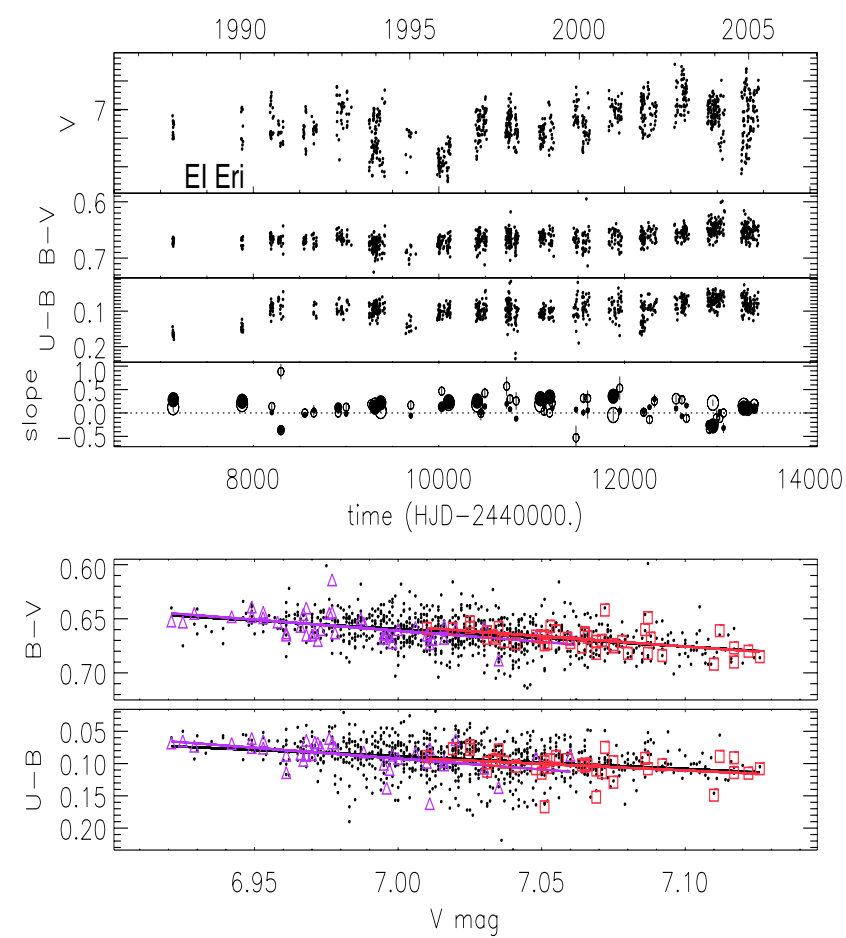

Fig. 4. As in Fig. 2, but for EI Eri and V1149 Ori.

anticorrelation between $U-B$ and $V$ data were found by Rodonò $\&$ Cutispoto (1992). We adopt for the hot and cool components the values of parameters proper for their spectral classes: $T_{\mathrm{h}}=5900 \mathrm{~K}, T_{\mathrm{c}}=5600 \mathrm{~K}, R_{\mathrm{h}}=1.1 R_{\odot}, R_{\mathrm{c}}=2.0 R_{\odot}$, $M_{\mathrm{h}} / M_{\mathrm{c}} \simeq 1.0, i=50^{\circ}$ (Strassmeier et al. 1993), and $\log g_{\mathrm{h}}$ $=4.5, \log g_{\mathrm{c}}=3.5 \mathrm{~cm} \mathrm{~s}^{-2}(\operatorname{Cox} 2000)$. We find that the variability of EI Eri arising from proximity and reflection effects is about $\Delta V_{\text {ellip }}=4.0 \times 10^{-3} \mathrm{mag}$ and $\Delta V_{\text {ref }}=4.3 \times 10^{-3} \mathrm{mag}$ (Morris \& Naftilan 1993).
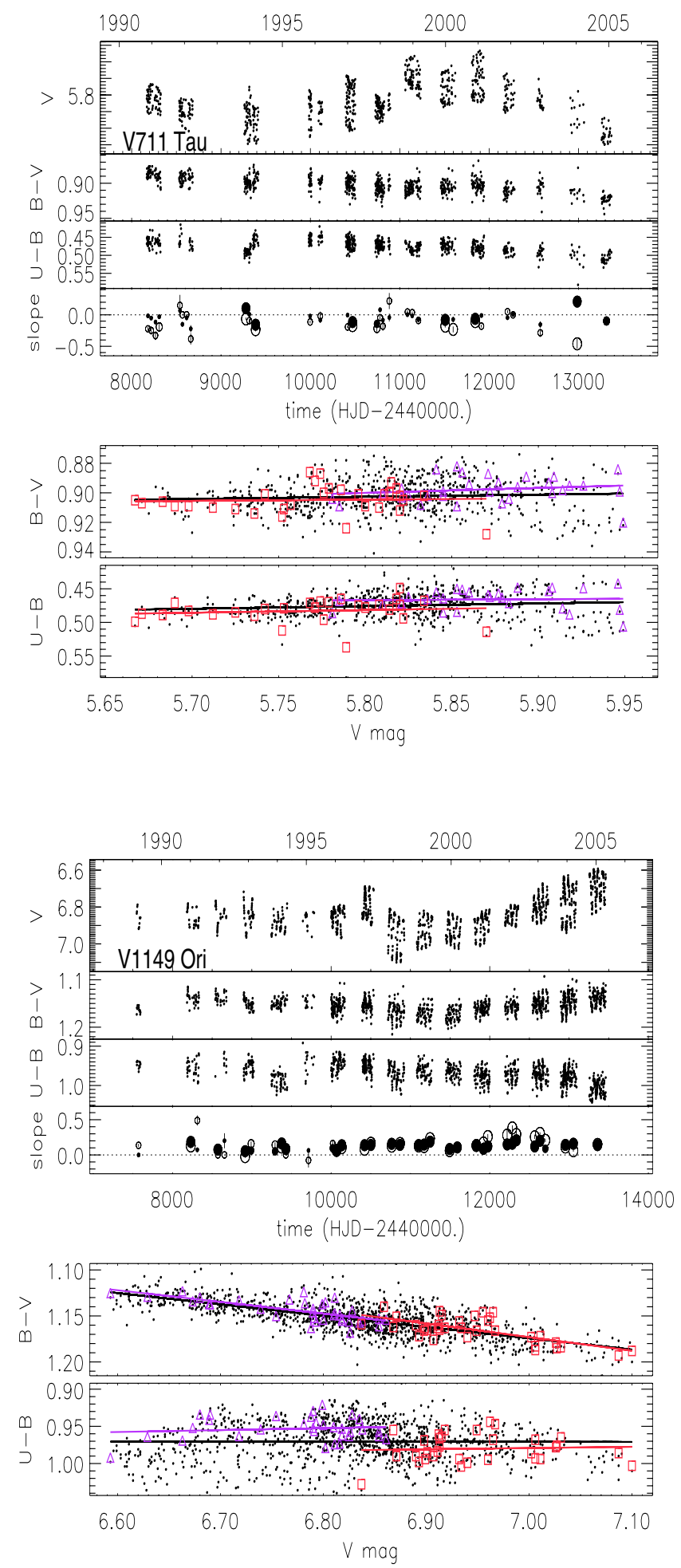

V1149 Ori (HD 37824) is an SB1 RS CVn-type variable with a primary K0 giant (Fekel \& Henry 2005) and colors from UBVRI photometry consistent with a $\mathrm{K} 2 / 3 \mathrm{III}+\mathrm{F} 8 \mathrm{~V}$ binary system (Cutispoto et al. 2001). V1149 Ori has an orbital period of $P_{\text {orb }}=53.57465^{\mathrm{d}}$ (Fekel et al. 1987) and a variable rotational photometric period with a mean value of $P_{\text {pho }}=53.12^{\mathrm{d}}($ Fekel \& Henry 2005). If granted the spectral classification by Cutispoto (1995), the observed photometric variability can be completely attributed to the giant component. The non-active earlier-type 


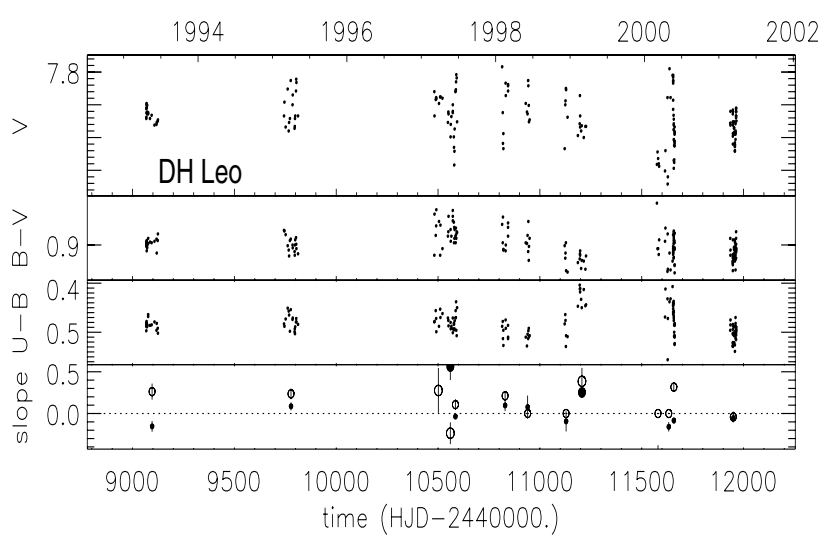

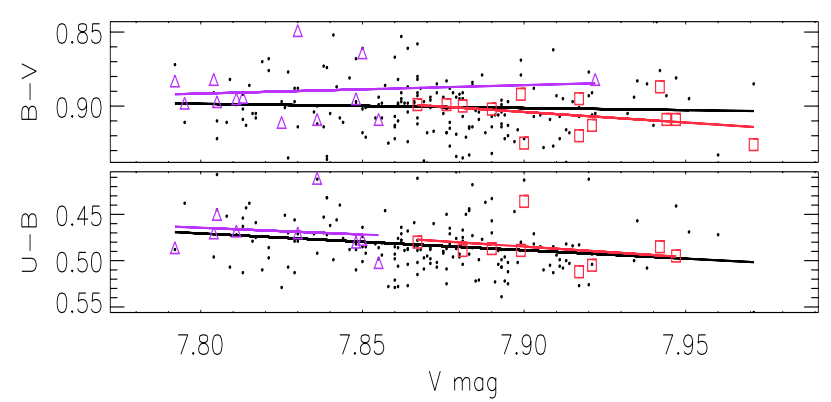

Fig. 5. As in Fig. 2, but for DH Leo and HU Vir.

component can give a significant flux contribution to the $U$ band where the total fluxes from both components tend to be comparable, as the magnetic activity makes the late-type component fainter. We adopt for the hot and cool components the values of parameters proper for their spectral classes: $T_{\mathrm{h}}=6200 \mathrm{~K}$, $T_{\mathrm{c}}=4600 \mathrm{~K}, R_{\mathrm{h}}=1.15 R_{\odot}, R_{\mathrm{c}}=12.6 R_{\odot}, M_{\mathrm{h}} / M_{\mathrm{c}} \simeq 0.90$, and $\log g_{\mathrm{h}}=4.5, \log g_{\mathrm{c}}=2.0 \mathrm{~cm} \mathrm{~s}^{-2}$ (Cox 2000) and an assumed value of $i=60^{\circ}$. We find that the variability of V1149 Ori arising from proximity and reflection effects is about $\Delta V_{\text {ellip }}=1.3 \times$ $10^{-3}$ mag and $\Delta V_{\text {ref }}=6.3 \times 10^{-4} \mathrm{mag}$ (Morris \& Naftilan 1993).

DH Leo (HD 86590) is a triple system consisting of a BY-Dra type $\mathrm{K} 0 \mathrm{~V}+\mathrm{K} 7 \mathrm{~V}$ binary with an orbital period of $P_{\text {orb }} \sim 1.070354^{\mathrm{d}}$ (Bolton et al. 1981) and a K5 V tertiary component (Barden 1984). The observed photometric variability is dominated by the $\mathrm{K} 0 \mathrm{~V}$ component, whose $U, B$ and $V$ total fluxes are larger than the fluxes from the $\mathrm{K} 5 \mathrm{~V}$ and $\mathrm{K} 7 \mathrm{~V}$. We adopt for the components the values of parameters proper for their spectral classes: $T_{\mathrm{h}}=5300 \mathrm{~K}, T_{\mathrm{c}}=4300 \mathrm{~K}, T_{3}=4600 \mathrm{~K}$, $R_{\mathrm{h}}=0.81 R_{\odot}, R_{\mathrm{c}}=0.65 R_{\odot}, R_{3}=0.68 R_{\odot}, M_{\mathrm{K} 0 \mathrm{~V}} / M_{\mathrm{K} 7 \mathrm{~V}} \simeq$ $1.3, i=80^{\circ}$ (Strassmeier et al. 1993), $\log g_{\mathrm{h}}=4.5, \log g_{\mathrm{c}}=4.5$, $\log g_{3}=4.5 \mathrm{~cm} \mathrm{~s}^{-2}$ (Cox 2000). We find that the variability of $\mathrm{DH}$ Leo arising from proximity and reflection effects is about $\Delta V_{\text {ellip }}=1.0 \times 10^{-3} \mathrm{mag}$ and $\Delta V_{\text {ref }}=2.8 \times 10^{-3} \mathrm{mag}$ (Morris \& Naftilan 1993).

HU Vir (HD 106225) is an SB1 RS CVn-type system with a K1 subgiant (Cutispoto 1998) and an orbital period of $P_{\text {orb }}=$ $10.38758^{\mathrm{d}}$ (Strassmeier 1994). The rotational photometric period of about $P_{\text {pho }}=10.41^{\mathrm{d}}$ is variable (Strassmeier et al. 1997). In our model we adopt as effective temperature, radius and surface gravity the values proper for the subgiant component: $T_{\mathrm{c}}=5000 \mathrm{~K}, R_{\mathrm{c}}=8 R_{\odot}, \log g_{\mathrm{c}}=3.5 \mathrm{~cm} \mathrm{~s}^{-2}(\operatorname{Cox} 2000)$. HU Vir will be treated in the analysis as a single star and proximity and reflection effects will be considered negligible.

RS CVn (HD 114519) is an eclipsing detached binary system consisting of a K2 subgiant and a F5 main-sequence star
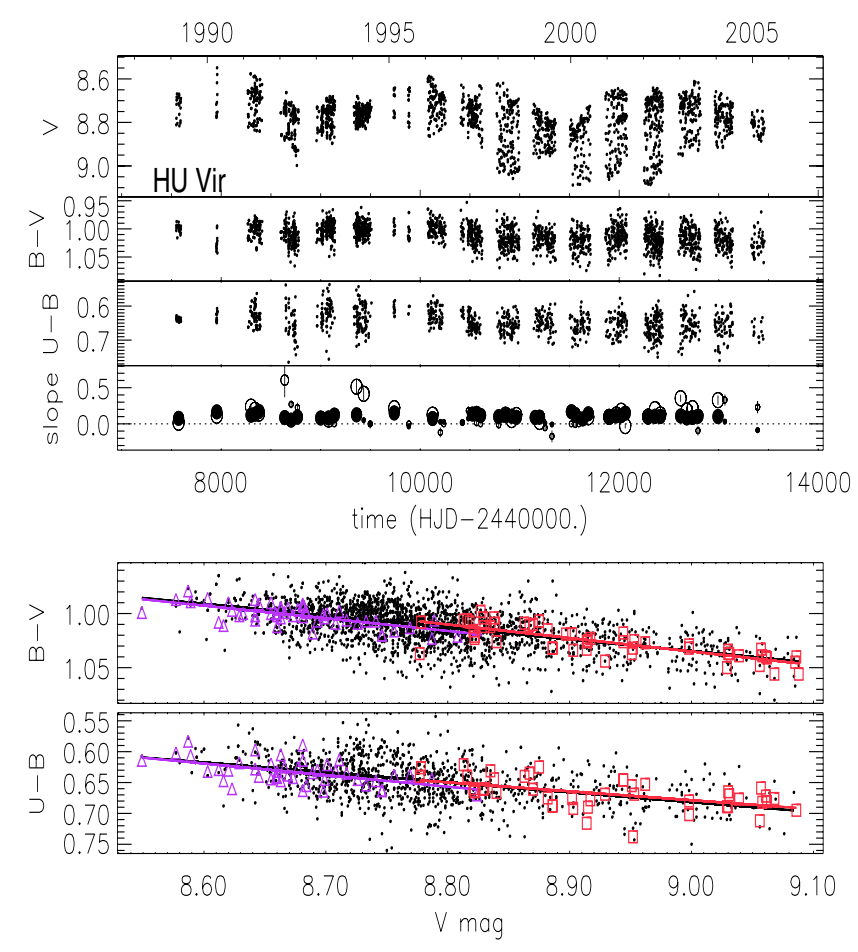

(Reglero et al. 1990). This system has a mean orbital period of $P_{\text {orb }}=4.797855^{\mathrm{d}}$ (Catalano \& Rodonò 1974). The observed photometric variability can be entirely attributed to the subgiant, which is the only magnetically active component. However, the earlier-type component has a slightly larger total flux in the $U$, $B$ and $V$ bands, which can give a significant contribution to the observed blueing as the brightness of the active component decreases (Aarum Ulvas \& Henry 2005). We adopt for the hot and cool component the values of parameters from Rodonò et al. (1995, 2001a): $T_{\mathrm{h}}=6300 \mathrm{~K}, T_{\mathrm{c}}=4600 \mathrm{~K}, R_{\mathrm{h}}=1.89 R_{\odot}$, $R_{\mathrm{c}}=3.85 R_{\odot}, M_{\mathrm{h}} / M_{\mathrm{c}}=0.96, i=85^{\circ}$, and $\log g_{\mathrm{h}}=4.5, \log g_{\mathrm{c}}=$ $3.5 \mathrm{~cm} \mathrm{~s}^{-2}$. We find that the variability of RS CVn arising from proximity and reflection effects is about $\Delta V_{\text {ellip }}=0.01 \mathrm{mag}$ and $\Delta V_{\text {ref }}=4.7 \times 10^{-3} \mathrm{mag}$ (Morris \& Naftilan 1993).

V775 Her (HD 175742) is an SB1 BY-Dra type variable consisting of a K0 and a K5/M2 main-sequence stars and with an orbital period of $P_{\text {orb }}=2.879395^{\mathrm{d}}$ (Imbert 1979). Although both components are expected to be magnetically active, due to their short rotation period and late-spectral type, the luminosity of the K0 component largely dominates the system's luminosity. We adopt for the hot and cool components values of parameters proper for their spectral classes: $T_{\mathrm{h}}=5300 \mathrm{~K}, T_{\mathrm{c}}=4000 \mathrm{~K}$, $R_{\mathrm{h}}=0.85 R_{\odot}, R_{\mathrm{c}}=0.60 R_{\odot}, M_{\mathrm{h}} / M_{\mathrm{c}} \simeq 1.3$, and $\log g_{\mathrm{h}}=4.5$, $\log g_{\mathrm{c}}=4.5 \mathrm{~cm} \mathrm{~s}^{-2}$ (Cox 2000) and an assumed value of $i=60^{\circ}$. We find that the variability of V775 Her arising from proximity and reflection effects is about $\Delta V_{\text {ellip }}=1.7 \times 10^{-4}$ mag and $\Delta V_{\text {ref }}=6.2 \times 10^{-4} \mathrm{mag}$ (Morris \& Naftilan 1993).

AR Lac (HD 210334) is an eclipsing detached binary system of RS-CVn type consisting of a G2 and a K2 subgiants (Hall 1976). AR Lac has an orbital period of about $P_{\text {orb }}=1.9832142^{\mathrm{d}}$ (Jetsu et al. 1997). The observed photometric variability can be mostly attributed to the K2 subgiant, due to the deeper convection zone with respect to the $\mathrm{G} 2$ component. Nonetheless, since both components have similar total fluxes in $U, B$ and $V$ bands, the hotter component may play some role in the observed color 

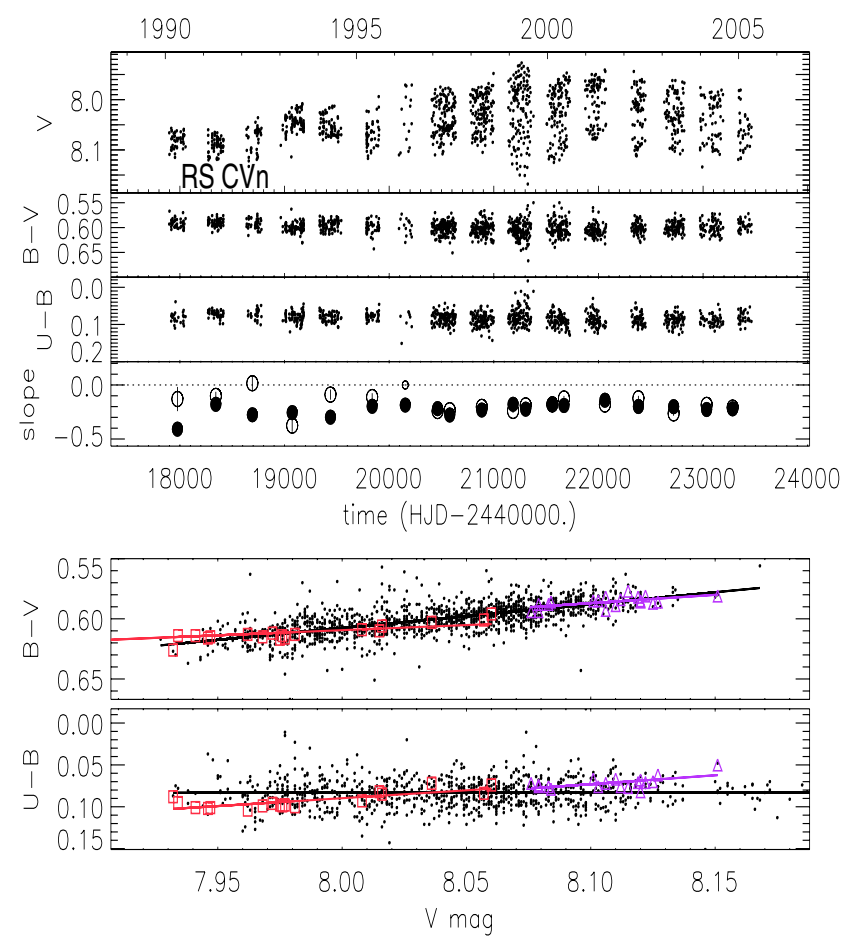

Fig. 6. As in Fig. 2, but for RS CVn and V775 Her.
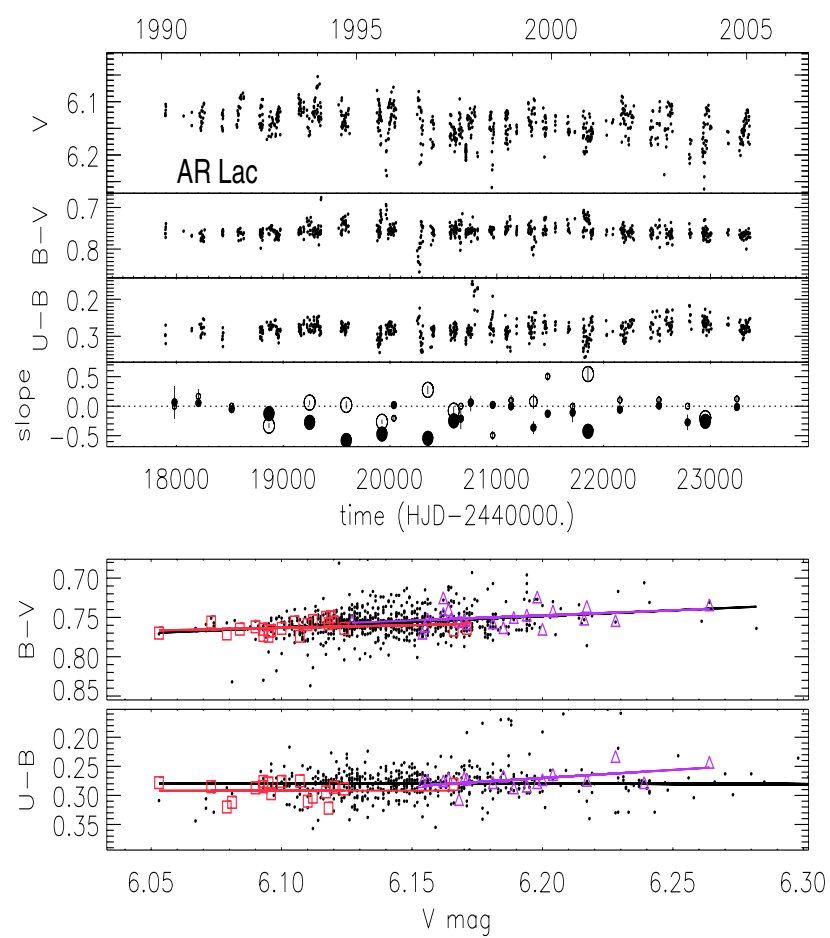

Fig. 7. As in Fig. 2, but for AR Lac and SZ Psc.

variations, as will be discussed in the following. We adopt for the hot and cool components the values of parameters from Lanza et al. (1998): $T_{\mathrm{h}}=5560 \mathrm{~K}, T_{\mathrm{c}}=4820 \mathrm{~K}, R_{\mathrm{h}}=1.51 R_{\odot}$, $R_{\mathrm{c}}=2.61 R_{\odot}, M_{\mathrm{h}} / M_{\mathrm{c}}=0.97, i=87^{\circ}$, and $\log g_{\mathrm{h}}=4.0, \log g_{\mathrm{c}}=$ $3.5 \mathrm{~cm} \mathrm{~s}^{-2}$. We find that the variability of AR Lac arising from proximity and reflection effects is about $\Delta V_{\text {ellip }}=0.027$ mag and $\Delta V_{\text {ref }}=0.010 \mathrm{mag}$ (Morris \& Naftilan 1993).

SZ Psc (HD 219113) is an eclipsing detached binary system belonging to the RS CVn class of variable stars and consisting of an F8 V/IV and a K1 subgiant (Hall 1976). This system has an
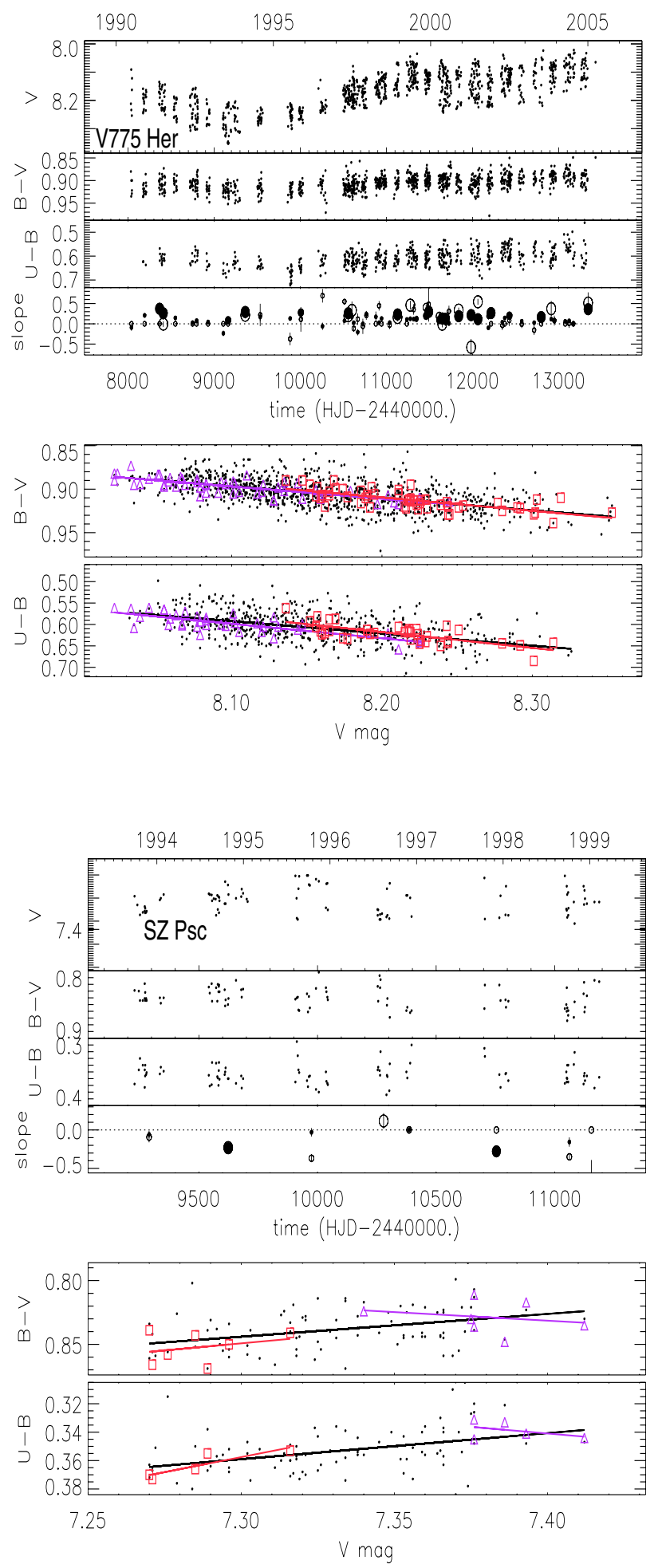

orbital period of $P_{\text {orb }}=3.9657889^{\mathrm{d}}$. The observed photometric variability arises from the subgiant component which is the only magnetically active component. However, the earlier-type component can give a significant flux contribution to the $U$ band as the magnetic activity makes the $\mathrm{K} 1$ component fainter. We adopt for the hot and cool components the values of parameters from Lanza et al. (2001) and Eaton \& Henry (2007): $T_{\mathrm{h}}=6100 \mathrm{~K}$, $T_{\mathrm{c}}=4900 \mathrm{~K}, R_{\mathrm{h}}=1.5 R_{\odot}, R_{\mathrm{c}}=6.0 R_{\odot}, M_{\mathrm{h}} / M_{\mathrm{c}}=0.76, i=69^{\circ}$, and $\log g_{\mathrm{h}}=4.20, \log g_{\mathrm{c}}=3.23 \mathrm{~cm} \mathrm{~s}^{-2}$. We find that the variability of SZ Psc arising from proximity and reflection effects is 
Table 2. $V$ magnitude, $B-V$ and $U-B$ colors of comparison (C) and check $(\mathrm{CK})$ stars.

\begin{tabular}{|c|c|c|c|c|c|c|}
\hline \multicolumn{2}{|c|}{ Program star } & \multicolumn{2}{|c|}{ C/CK star } & \multirow{2}{*}{$\begin{array}{c}\begin{array}{c}V \\
(\mathrm{mag})\end{array} \\
6.04\end{array}$} & \multirow{2}{*}{\multicolumn{2}{|c|}{$\begin{array}{cr}\begin{array}{c}B-V \\
(\mathrm{mag})\end{array} & \begin{array}{c}(\mathrm{mag}) \\
\end{array} \\
1.08 & 1.02\end{array}$}} \\
\hline 1 & $\overline{A R} P_{S c}$ & HD 7446 & $(\mathrm{C})$ & & & \\
\hline & & HD 7804 & (CK) & 5.16 & 0.07 & 0.03 \\
\hline \multirow[t]{4}{*}{2} & VY Ari & HD 17572 & (C) & 6.72 & 0.37 & -0.03 \\
\hline & & HD 17329 & (CK1) & 7.93 & 0.76 & 0.28 \\
\hline & & HD 17395 & (CK2) & 8.45 & 0.59 & 0.06 \\
\hline & & HD 16187 & (CK3) & 6.05 & 1.05 & 0.88 \\
\hline \multirow[t]{3}{*}{3} & UX Ari & HD 20825 & (C) & 5.55 & 1.10 & 0.92 \\
\hline & & HD 20618 & (CK1) & 5.91 & 0.86 & 0.45 \\
\hline & & HD 20644 & (CK2) & 4.47 & 1.55 & 1.86 \\
\hline \multirow[t]{4}{*}{4} & V711 Tau & HD 22796 & (C) & 5.55 & 0.94 & 0.67 \\
\hline & & HD 22819 & (CK1) & 6.10 & 0.99 & 0.78 \\
\hline & & HD 23413 & (CK2) & 5.56 & 1.42 & 1.72 \\
\hline & & HD 22484 & (CK? & 4.29 & 0.57 & 0.05 \\
\hline \multirow[t]{4}{*}{5} & EI Eri & HD 25852 & (C) & 7.83 & 1.01 & 0.78 \\
\hline & & HD 25954 & (CK1) & 7.53 & 1.24 & 1.36 \\
\hline & & HD 26237 & & 7.17 & 0.03 & 0.03 \\
\hline & & HD 26409 & (CK & 5.44 & 0.94 & 0.67 \\
\hline \multirow[t]{4}{*}{6} & V1149 Ori & HD 37741 & (C) & 8.19 & 1.07 & 0.87 \\
\hline & & HD 38145 & (CK1) & 7.91 & 0.33 & 0.06 \\
\hline & & HD 38529 & (CK2) & 5.93 & 0.78 & 0.48 \\
\hline & & HD 37984 & (CK3) & 4.91 & 1.17 & 1.10 \\
\hline \multirow[t]{4}{*}{7} & DH Leo & HD 86132 & (C) & 8.17 & 0.96 & 0.65 \\
\hline & & HD 85428 & (CK1) & 7.78 & 1.24 & 1.33 \\
\hline & & HD 88008 & (CK & 8.48 & 0.74 & 0.26 \\
\hline & & HD 85376 & (CK3) & 5.30 & 0.22 & 0.09 \\
\hline \multirow[t]{4}{*}{8} & HU Vir & HD 106270 & (C) & 7.59 & 0.74 & 0.30 \\
\hline & & HD 105796 & (CK1) & 8.05 & 1.07 & 0.96 \\
\hline & & D 105759 & $(\mathrm{CK}$ & 6.55 & 0.22 & 0.07 \\
\hline & & HD 106516 & (CK3) & .12 & 0.46 & -0.14 \\
\hline \multirow[t]{6}{*}{9} & RS CVn & HD 114778 & (C) & 8.40 & 0.47 & -0.04 \\
\hline & & HD 114838 & (CK & 8.08 & 0.53 & 0.01 \\
\hline & & HD 114863 & (CK & 8.54 & 0.57 & 0.02 \\
\hline & & $\mathrm{BD}+362347$ & (CK & 9.91 & 0.50 & -0.09 \\
\hline & & HD 113797 & $(\mathrm{CK}$ & 5.22 & -0.07 & -0.19 \\
\hline & & HD 114357 & (CK5) & 6.02 & 1.17 & 1.16 \\
\hline \multirow[t]{5}{*}{10} & V775 Her & HD 337271 & (C) & 8.74 & 1.12 & 1.00 \\
\hline & & HD 337275 & (CK1 & 8.55 & 1.25 & 1.30 \\
\hline & & HD 178187 & (CK & 5.85 & 0.09 & 0.19 \\
\hline & & HD 174160 & (CK & 6.22 & 0.48 & 0.02 \\
\hline & & D 175492 & & 4.60 & 0.78 & 0.48 \\
\hline \multirow[t]{4}{*}{11} & AR Lac & HD 208728 & (C) & 6.81 & 1.19 & 0.85 \\
\hline & & D 209945 & (CK1) & .10 & 1.58 & 1.95 \\
\hline & & HD 209219 & (CK2) & 7.40 & 1.35 & 1.56 \\
\hline & & & & .45 & 0.57 & 0.04 \\
\hline \multirow[t]{3}{*}{12} & SZ Psc & HD 219018 & (C) & 7.74 & 0.64 & 0.15 \\
\hline & & D 219150 & & 7.20 & 0.40 & -0.07 \\
\hline & & HD 218527 & (CK2) & 5.46 & 0.91 & 0.56 \\
\hline \multirow[t]{3}{*}{13} & II Peg & HD 224016 & (C) & 8.52 & 0.78 & 0.43 \\
\hline & & HD 223461 & (CK1) & 5.96 & 0.20 & 0.05 \\
\hline & & HD 224895 & (CK2) & 6.81 & 1.21 & 1.17 \\
\hline \multirow[t]{4}{*}{14} & BY Dra & HD 172268 & (CK1) & 7.89 & 1.24 & 1.31 \\
\hline & & HD 172468 & (CK2) & 7.52 & 1.25 & 1.16 \\
\hline & & HD 169028 & $(\mathrm{CK}$ & 6.29 & 1.10 & 1.08 \\
\hline & & HD 172883 & (CK4) & 5.98 & -0.07 & -0.23 \\
\hline
\end{tabular}

about $\Delta V_{\text {ellip }}=0.012 \mathrm{mag}$ and $\Delta V_{\text {ref }}=3.3 \times 10^{-3} \mathrm{mag}$ (Morris $\&$ Naftilan 1993).

II Peg (HD 224085) is an SB1 RS CVn-type binary system with a primary K2 IV component (Rucinski 1977). It has a variable rotational photometric period of about $P_{\text {pho }}=6.720^{\mathrm{d}}$ (Rodonò et al. 2000). We adopt as effective temperature the values from Rodonò et al. (2000), and radius and surface gravity values proper for its spectral class: $T_{\mathrm{h}}=4600 \mathrm{~K}, R_{\mathrm{h}}=8 R_{\odot}$,
Table 3. Total number of averaged observations in the $V, B$ and $U$ band, total number of light curves and interval of time of the photometric monitoring.

\begin{tabular}{lrrrrr}
\hline \hline Program star & $V$ & $B$ & $U$ & L.C. & Time range \\
\hline AR Psc & 620 & 595 & 555 & 35 & $1987-2004$ \\
VY Ari & 980 & 966 & 904 & 40 & $1991-2004$ \\
UX Ari & 1169 & 1157 & 1088 & 37 & $1990-2004$ \\
V711 Tau & 747 & 733 & 669 & 31 & $1990-2004$ \\
EI Eri & 961 & 934 & 829 & 43 & $1987-2005$ \\
V1149 Ori & 1247 & 1226 & 1114 & 35 & $1989-2004$ \\
DH Leo & 184 & 184 & 179 & 13 & $1993-2001$ \\
HU Vir & 2182 & 2021 & 1006 & 52 & $1989-2004$ \\
RS CVn & 1900 & 1019 & 969 & 19 & $1990-2004$ \\
V775 Her & 1105 & 1048 & 564 & 54 & $1990-2004$ \\
AR Lac & 2348 & 698 & 620 & 23 & $1990-2004$ \\
SZ Psc & 185 & 84 & 80 & 8 & $1993-1998$ \\
II Peg & 1233 & 1193 & 1049 & 43 & $1992-2004$ \\
BY Dra & 734 & 725 & 391 & 42 & $1990-2004$ \\
\hline
\end{tabular}

$\log g_{\mathrm{h}}=3.50 \mathrm{~cm} \mathrm{~s}^{-2}$. II Peg will be treated in the analysis as a single star and proximity and reflection effects will be considered negligible.

BY Dra (HD 234677) is an SB2 BY Dra-type binary system consisting of two M0 main-sequence stars (Rodonò \& Cutispoto 1992). This system has an orbital period of $P_{\text {orb }}=5.976^{\mathrm{d}}$ (Bopp \& Evans 1973) which significantly differs from the rotational photometric period of about $P_{\text {pho }}=3.836^{\mathrm{d}}$ (Rodonò et al. 1983). Both components are expected to equally contribute to the observed photometric variability, their rotation rate and spectral type being equal. We adopt for the components the values of parameters proper for their spectral classes: $T_{\mathrm{h}}=3700 \mathrm{~K}$, $R_{\mathrm{h}}=0.60 R_{\odot}, M_{\mathrm{h}} / M_{\mathrm{c}}=1.0$, and $\log g_{\mathrm{h}}=4.50 \mathrm{~cm} \mathrm{~s}^{-2}(\mathrm{Cox}$ 2000 ) and an assumed value of $i=60^{\circ}$. We find that the variability of BY Dra arising from proximity and reflection effects is about $\Delta V_{\text {ellip }}=5.5 \times 10^{-5} \mathrm{mag}$ and $\Delta V_{\text {ref }}=4.0 \times 10^{-4} \mathrm{mag}$ (Morris \& Naftilan 1993).

The brightest $V$ magnitude, maximum $V$-band light curve amplitude and average $B-V$ and $U-B$ colors of the program stars are listed in Table 1.

\section{Observations}

The photometric observations were collected by two APTs: the Phoenix-25 since 1988, and the APT80/1 since late 1992. The Phoenix-25 is a $25-\mathrm{cm}$ telescope operated under the "rent-astar" service by the Franklin \& Marshall College at Washington Camp (AZ, USA). It feeds a single-channel photon counting photometer, equipped with an uncooled 1P21 photomultiplier and standard $U B V$ filters (Boyd et al. 1984; Baliunas et al. 1985). The APT80/1 is a $80-\mathrm{cm}$ telescope located at the M. G. Fracastoro station of OAC on Mt. Etna (1725 m a.s.l.) that feeds a single channel charge-integration photometer, equipped with an uncooled Hamamatsu R1414 SbCs photomultiplier and standard $U B V$ filters (Rodonò \& Cutispoto 1992; Messina 1998).

The Phoenix-25 observed the program stars differentially with respect to the comparison (C) and check (CK1) stars listed in Table 2. A detailed description of the observation and reduction procedures for the data collected with this telescope can be found in Rodonò \& Cutispoto (1992).

The APT80/1 observed all the program stars differentially with respect to a larger set of comparison stars (all stars in Table 2) including those observed by the Phoenix-25. 

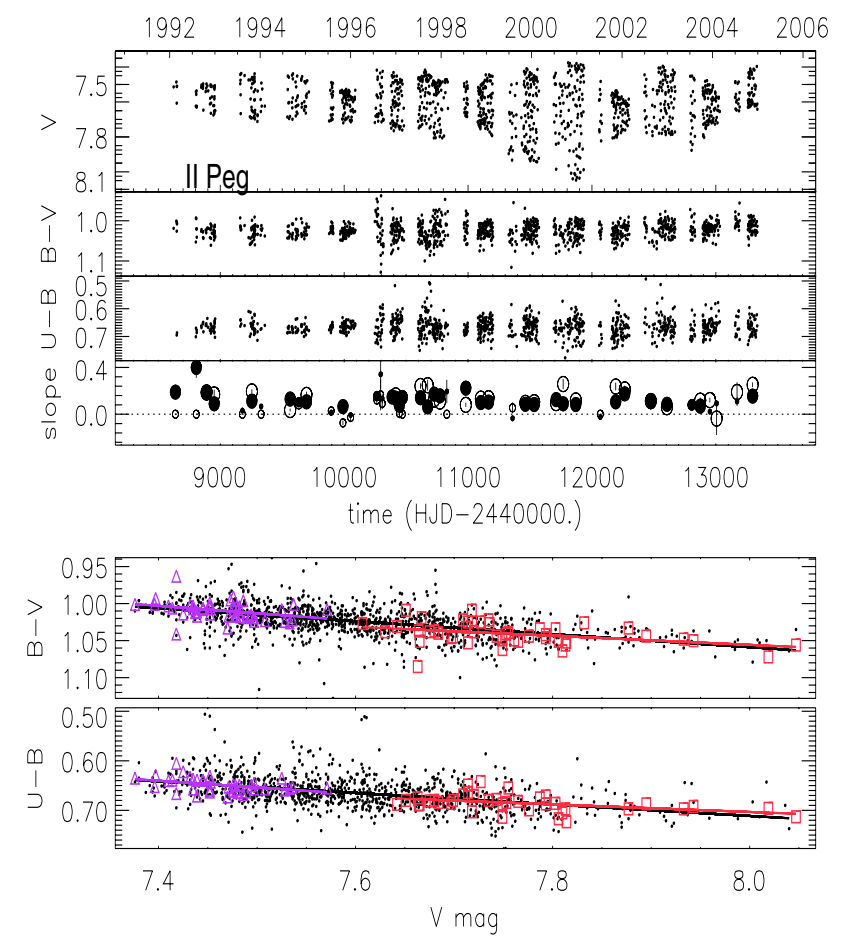

Fig. 8. As in Fig. 2, but for II Peg and BY Dra.

The integration time in $U, B$ and $V$ filters was set to 15,10 and $10 \mathrm{~s}$, respectively, and the observing sequence was $n-c-c k 1-$ $c-v-v-v-c-v-v-v-c-c k 2-c-n$, where the symbol $n$ denotes the bright navigation star, which is the first star of the group the APT80/1 hunts. The sky background was measured at a fixed position near each star.

Differential magnitudes from both telescopes were corrected for atmospheric extinction and transformed into the standard Johnson $U B V$ system. The transformation coefficients were determined quarterly by observing selected samples of standard stars. Due to the relatively short duration of an observing sequence $(\simeq 30 \mathrm{~min})$, differential values were averaged to obtain one single average point. The complete dataset presented in this paper consists of about 16000,12600 and 10000 average points in the $V, B$ and $U$ filters (see Table 3). After transformation into the standard system the achieved accuracy of $V$ magnitude, $B-V$ and $U-B$ color indices is of the order of $0.008,0.010$ and $0.012 \mathrm{mag}$, respectively, for the faintest stars $(V \simeq 8.5 \mathrm{mag})$. A comparison of the standard deviations of $c k-c$ and $v-c$ differential magnitudes shows that the comparison stars have remained constant within the observation accuracy.

We could homogenize the observations coming from two different telescopes, since they observed a common set of comparison and check stars for many years.

In order to further extend our time series, in the following analysis we made use also of the UBV observations of AR Psc, EI Eri, V1149 Ori and HU Vir collected with the 50-cm telescope at ESO (La Silla, Chile) published by Cutispoto (1990, 1992, 1993). For the eclipsing binary stars AR Lac, RS CVn and SZ Psc, we will consider only the out-of-eclipse observations, our analysis being focused only on the magnitude and color variations arising from the presence of photospheric inhomogeneities. In order to determine the out-of-eclipse phases, we also took into account the orbital period variations.

In order to better investigate the evolution of shape, amplitude and mean magnitude shown by the light curves, the whole
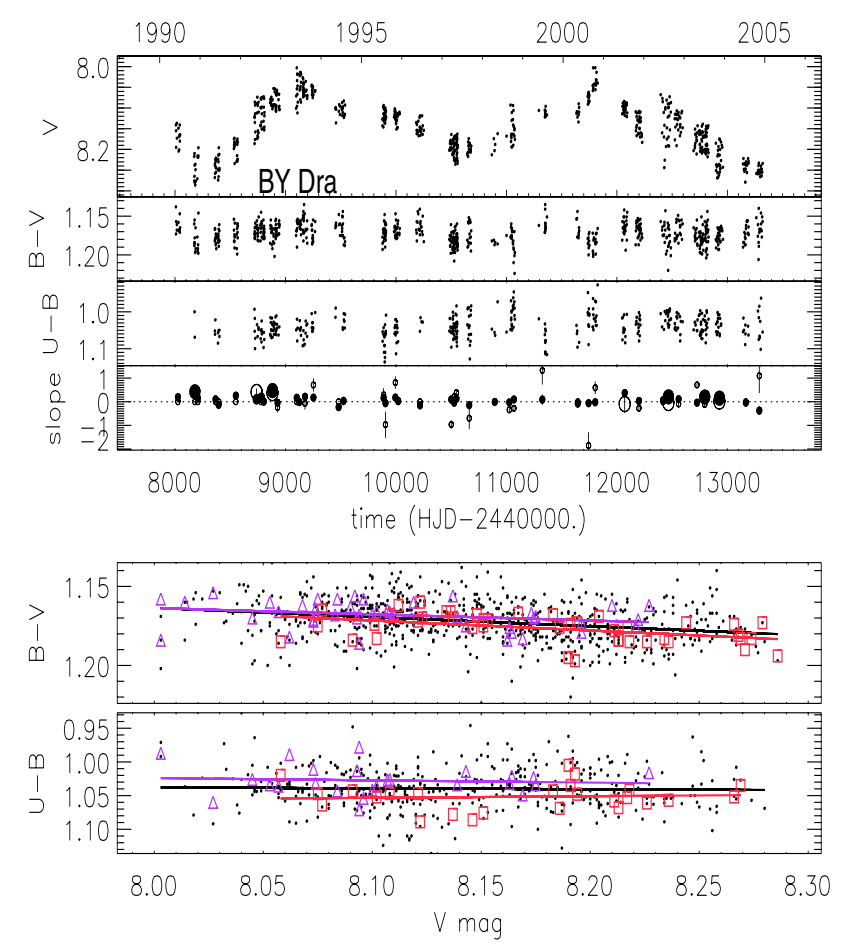

data set of each program star was subdivided into a number of light curves. The division was made by selecting time intervals during which the star displayed a stable flux modulation, i.e. no significant differences (smaller than $\sim 0.01-0.02 \mathrm{mag}$ ) between observations falling close to each other within 0.01-0.02 dex in rotational phase. That division allowed us to obtain from a minimum of 8 light curves in the case of SZ Psc to a maximum of 54 light curves in the case of V775 Her. In Fig. 1 we plot, as an example, one of the 37 light curves in which the complete series of observations of UX Ari has been divided. $V$ magnitude, $B-V$ and $U-B$ colors for the mean epoch 1993.82 are plotted vs. rotational phase in the top three panels of Fig. 1. The most relevant properties of the light curves of each program star are listed in the on-line Table 4. Specifically, we give: mean epoch of observations (Mean Epoch), mean ( $\left.\mathrm{HJD}_{\text {mean }}\right)$, initial (HJD $\left.\mathrm{Hin}_{\mathrm{i}}\right)$ and final $\left(\mathrm{HJD}_{\mathrm{end}}\right)$ heliocentric Julian day, number of points in the light curve $\left(N_{\mathrm{m}}\right)$, brightest $V$ magnitude $\left(V_{\min }\right)$ and light curve peak-to-peak amplitude in the $V$ band $(\Delta V), B-V$ and $U-B$ colors, standard deviation $\left(\sigma_{v}\right)$ of $v-c$ and $\left(\sigma_{c k 1-c}\right)$ of $c k 1-c$ differential observations, and the telescope used to make the observations.

With the exception of SZ Psc and DH Leo, all the targets were observed in many epochs almost contemporaneously by the two mentioned telescopes. The difference in longitude between the two observation sites allowed us to observe the same stars almost continuously for nine additional hours; thus we were able to obtain for the short-period stars a set of light curves well covered in rotational phase.

\section{4. $V$ magnitude versus colors variations}

In Figs. 2-8 we plot the complete series of $V$ magnitudes (top panel), $B-V$ and $U-B$ colors (second and third panel from top) of our program stars. To date, this $U B V$ database is among the longest and most homogeneous for the program close binary systems. Although their long- and short-term photometric 
behaviours have been investigated in a number of papers, unlike previous works mostly based only on $V$-band data, our data allow us to also investigate the color behaviour on both short and long timescales and using a very homogeneous data sample.

\subsection{Short-term (rotational) variation}

The magnitude and color variations generally shown by active stars and related to magnetic activity have different time scales: the star's rotation modulates the visibility of asymmetrically distributed photospheric temperature inhomogeneities and, as a consequence, gives rise to magnitude and color variations with the same period of the star's rotation period. Within a few stellar rotations, the inhomogeneities grow and decay change either the amplitude or shape of the flux rotational modulation, as well as the mean brightness level. On a longer timescale, variations of the inhomogeneities total area and their latitudinal migration, which are both related to the presence of starspot activity cycles on a differentially rotating star, cause additional magnitude and color variations (Messina et al. 2004). In order to separate the effects of rotation from those arising from ARGD and activity cycles, the complete time series of observations of each program star has been divided in a number of light curves, as mentioned in the previous section, i.e., each corresponding to an interval during which the star displayed a stable flux modulation.

For each light curve (see Fig. 1) we have carried out correlation and regression analyses between colors and magnitude variations ( $B-V$ and $U-B$ vs. $V$, and $U-B$ vs. $B-V$ ), by computing correlation coefficients $(r)$, their significance level $(\alpha)$, and slopes of linear fits (see lower panels of Fig. 1). The significance level $\alpha$ represents the probability of observing a value of the correlation coefficient larger than $r$ for a random sample having the same number of observations and degrees of freedom (Bevington 1969). Correlation analysis allows us to investigate the origin of magnitude and color variations. For example, if these variations originate from a single spot or group of small spots, as well as if they originate from two different, but spatially and temporally correlated types of inhomogeneities, e.g. cool spots and hot faculae, we expect these quantities to be correlated. On the contrary, a poor correlation or its absence will tell us that magnitude and colors are affected by at least two mechanisms, which are operating independently from each other, either spatially or temporally. As will be discussed in Sect. 6, the presence of magnetic activity in the fainter stellar component of the system may also play a role in decreasing the expected correlation. The regression analysis is also important to infer relevant information on the properties of photospheric inhomogeneities, since their average temperature mostly determines the slope of the fits. Indeed, surface inhomogeneities with different areas but constant temperature will determine magnitude and color variation of different amplitude but with a rather constant ratio. We have computed the slope $b$ of the linear fit $y=a+b x$ to $B-V$ vs. $V\left(b_{b v}\right)$, to $U-B$ vs. $V\left(b_{u b}\right)$ and to $U-B$ vs. $B-V\left(b_{u b v}\right)$ for each light curve; $b_{b v}$ and $b_{u b}$ values are listed in the on-line Table 5 and plotted in Figs. 2-8 (fourth panel from top) with filled and open circles, respectively. The symbol size indicates different significance levels $\alpha$ of the correlation coefficients $r$ : the largest size is for $\alpha \leq 0.05$, middle size for $0.05<\alpha \leq 0.1$, and smallest size for $\alpha>0.1$. The values of the average slopes $\left\langle b_{b v}\right\rangle,\left\langle b_{u b}\right\rangle$ and $\left\langle b_{u b v}\right\rangle$, uncertainty, number of light curves used to make the average (only curves with $\alpha<0.1$ ) and the slope minimum and maximum values are listed in the on-line Table 6 and plotted in Fig. 9 (top panel) with filled bullets, open bullets and diamonds, respectively. Targets are just ordered by decreasing value of the average slope.

Correlation and regression analyses have given three important results:

i) magnitude and color variations are found to be correlated to each other only in a fraction of light curves. The percentage of light curves in which $V$ magnitude and color variations are significantly correlated $(\alpha<0.1)$ is over $60 \%$ for VY Ari, II Peg, V1149 Ori, HU Vir, UX Ari and RS CVn. These stars will be referred to as color-correlated stars. The percentage is smaller than $40 \%$ for BY Dra, V775 Her, EI Eri, DH Leo, AR Lac, V711 Tau, AR Psc, SZ Psc. These stars will be referred to as color-uncorrelated stars. The $B-V$ and $U-B$ vs. $V$ variations are generally found to be correlated more frequently than the $U-B$ vs. $B-V$ variations;

ii) the values of $\left\langle b_{b v}\right\rangle$ and $\left\langle b_{u b}\right\rangle$, as computed by considering values for which $\alpha<0.1$, are positive for 8 program stars: BY Dra, VY Ari, V775 Her, II Peg, V1149 Ori, HU Vir, EI Eri, DH Leo. This means that along the star's rotation the fainter the star the redder its $B-V$ and $U-B$ colors. These stars will be referred to as reddening stars. Such a behaviour is consistent with a rotational flux modulation dominated by cool spots. The values of $\left\langle b_{b v}\right\rangle$ and $\left\langle b_{u b}\right\rangle$ are negative for 6 program stars: AR Lac, V711 Tau, RS CVn, UX Ari, AR Psc, SZ Psc. This means that along the star's rotation the fainter the star the bluer its $B-V$ and $U-B$ colors. These stars will be referred to as blueing stars. Such a different behaviour may be consistent with a rotational flux modulation dominated either by cool spots, whose negative flux contribution dominates the $V$-band variation, and by bright faculae, whose positive flux contribution dominates the $B$ and $U$-band variations. However, a flux contribution to the $B$ and $U$ bands by an earlier-type stellar companion may also cause a similar blueing, as will be discussed in Sect. 5;

iii) the values of the $b_{b v}$ and $b_{u b}$ slopes generally vary from light curve to light curve, often within a significant range of values (see the on-line Table 5), indicating that the average temperature of surface inhomogeneities is variable.

Although DH Leo has a negative slope, its associated uncertainty is large. As better shown in the following, it behaves like a reddening star. Although the $b_{u b v}$ value is expected to be positive for both reddening and blueing stars, it is found to be negative more frequently in V711 Tau, AR Lac, AR Psc, BY Dra (see on-line Table 5). For instance, we find than $b_{u b v}$ is negative when the $B-V$ and $U-B$ vs. $V$ variations are poorly correlated $(\alpha>0.1)$. In Sect. 6 we will show that the presence of an active fainter companion or the presence of a facular activity uncorrelated to the spot activity on the rotation time scale may be the reason for the negative slope of $b_{u b v}$.

\subsection{Long-term (cyclical) variation}

In order to investigate the relation between color-magnitude and color-color variations arising from activity cycles or long-term trends we have made correlation and linear regression analyses with the complete series of data (small dots in the lower panels of Figs. 2-8). Correlation coefficients $(r)$, significance levels $(\alpha)$, values of the fit slope $\left(b_{b v-\text { long }}, b_{u b-\text { long }}\right.$ and $\left.b_{u b v-\text { long }}\right)$, and number $(N)$ of data used to compute the fits are listed in the on-line Table 7 . The slopes $b_{b v-\text { long }}, b_{u b-\text { long }}$ and $b_{u b v-\text { long }}$ are plotted in the second panel of Fig. 9 as filled bullets, open bullets and diamonds, respectively. We have made separate fits also 


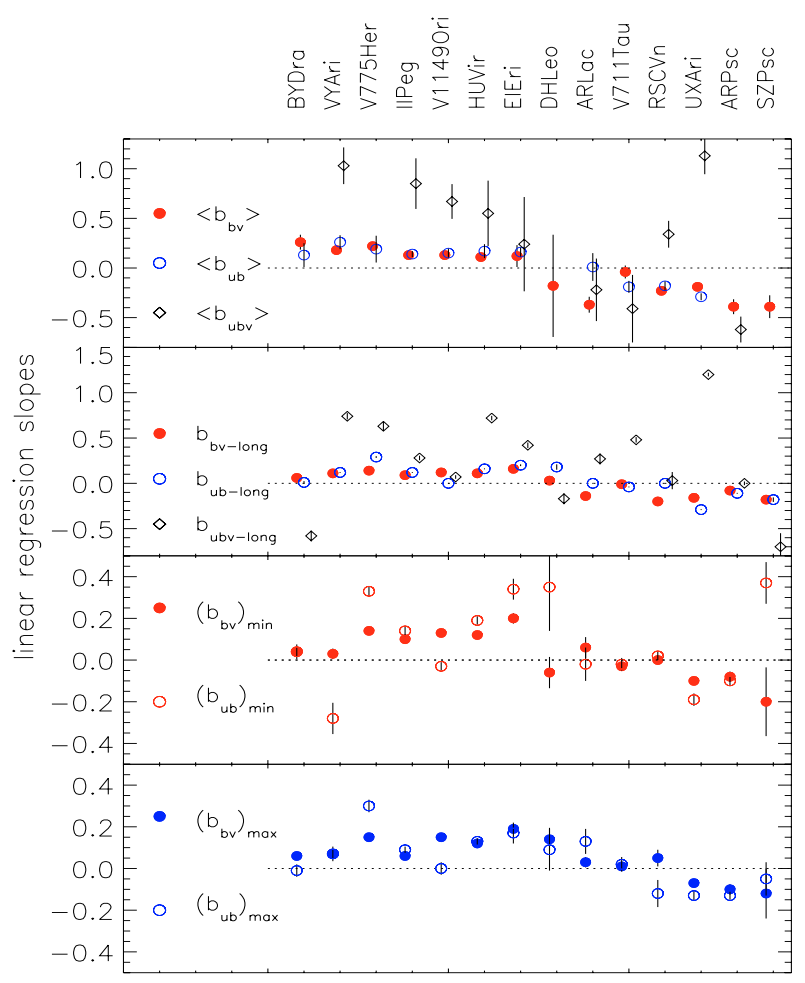

program stars

Fig. 9. Average slopes of the color-magnitude and color-color relations $(\alpha<0.1)$ arising from rotational modulation (top panel) and from activity cycles or long-term trends (second panel). Slopes of the relations between brightest/bluest (third panel) and between faintest/reddest light curves values (fourth panel).

to the brightest and bluest values $V_{\min },(B-V)_{\min },(U-B)_{\min }$ of each light curve (open triangles in lower panels of Figs. 2-8) and to the faintest and reddest values $V_{\max },(B-V)_{\max },(U-B)_{\max }$ (open squares) and computed the slope values $\left(b_{b v}\right)_{\min },\left(b_{u b}\right)_{\min }$ and $\left(b_{b v}\right)_{\max },\left(b_{u b}\right)_{\max }$. These slopes are also listed in the on-line Table 7 and plotted in the third and fourth panel of Fig. 9. Here we remind that the brightest magnitude and color values depend on those surface inhomogeneities evenly distributed along the stellar longitude, whereas the faintest values depend on evenly plus unevenly distributed inhomogeneities. We have also investigated the dependence of the slope values on the mean $\mathrm{V}$ magnitude, which is related to the phase of the starspot cycle. In a preliminary investigation, we considered all the slope values, independently of their significance level, and afterwards a subset of values with high significance $(\alpha \leq 0.1)$. The linear fits in both cases are generally in agreement, with the exclusion of AR Psc and AR Lac for which the number of significant values turned out to be small. The results of our investigation are plotted in Fig. 10, where the symbol size indicates the significance of the correlation coefficient as in Figs. 2-8, whereas different symbols indicate different ranges of the correlation coefficients: filled circles for $0<r \leq 0.2$, asterisks for $0.2<r \leq 0.4$, diamonds for $0.4<r \leq 0.6$, squares for $r \geq 0.6$.

Our regression analysis has given the following important results:

i) our program stars show on long timescales the same behaviour found in the rotation timescale: when activity cycles and/or long-term trends make the star's brightness fainter, all reddening stars become redder, whereas all blueing stars become bluer. Exceptions are V711 Tau, whose long-term $U-B$ and $B-V$ color variations are found to be uncorrelated with the $V$ mag variations and AR Lac, RS CVn and V1149 Ori whose $U-B$ long-term color variations are poorly correlated with the $V$ mag variations. For instance, in the case of V1149 Ori only data from 2004/2005 make the value of correlation coefficient close to zero;

ii) the reddening slopes related to the brightest values and arising from evenly distributed inhomogeneities are similar to the reddening slopes related to the faintest values and arising from evenly plus unevenly distributed inhomogeneities. An exception is VY Ari, whose brightest/bluest slope is negative, instead of being positive. For instance, for the blueing stars the brightest light curve values are found to be correlated with the reddest values and the faintest light curve values with the bluest. In other words, as the brightest light curve magnitude faints, the star becomes bluer, and when the most spotted hemisphere is in view the star becomes even bluer. We note that in the case of BY Dra and SZ Psc the color-color long-term variations, differently than expected, are anti-correlated;

iii) the values of the $b_{b v}$ slope of reddening stars is rather independent or slightly decreasing at increasing values of the mean $V$ magnitude. The values of the $b_{b v}$ slope of blueing stars becomes more negative (i.e. due to larger color variations) at increasing values of the mean $V$ magnitude. Very cool inhomogeneities do not produce color variation; on the contrary, the warmer the inhomogeneities the larger their color variations. Therefore, when our program stars approach the maximum level of starspot activity, the average temperature of inhomogeneities seems to be constant or slightly decreasing in reddening stars, whereas it seems to be increasing in blueing stars. Such an increase may arise from an increased flux contribution by hot faculae, as well as by the earlier-type component. Only the blueing star SZ Psc and the reddening star EI Eri deviate from this behaviour (see Fig. 10). On the other hand, a color variation may arise from the difference of limb-darkening between the most and the least spotted stellar hemisphere, depending on the average latitude in which spots are located.

\section{A simple modelling approach}

The analysis carried out so far is not enough to understand whether the behaviour shown by blueing stars arises from an enhanced flux contribution to the $B$ and $U$ bands by hot faculae or by the presence of the earlier-type component in the binary system, whose relative flux contribution becomes larger when the active late-type component is made fainter by magnetic activity. Additional information on the possible origin of the observed blueing is derived here by adopting a first-order modelling. An accurate modelling of the light and color curves of our program stars by using Maximum Entropy and Tikhonov regularization criteria will be carry out later to derive the area and temperature of active regions as well as their evolution over time (see, e.g. Lanza et al. 2006).

\subsection{Model}

We use the approach proposed by Dorren (1987) to model the amplitudes of the observed $V$ magnitude, $B-V$ and $U-B$ color variations arising from the difference of fluxes in the $U, B$ and $V$ bands between opposite hemispheres of the active component. 

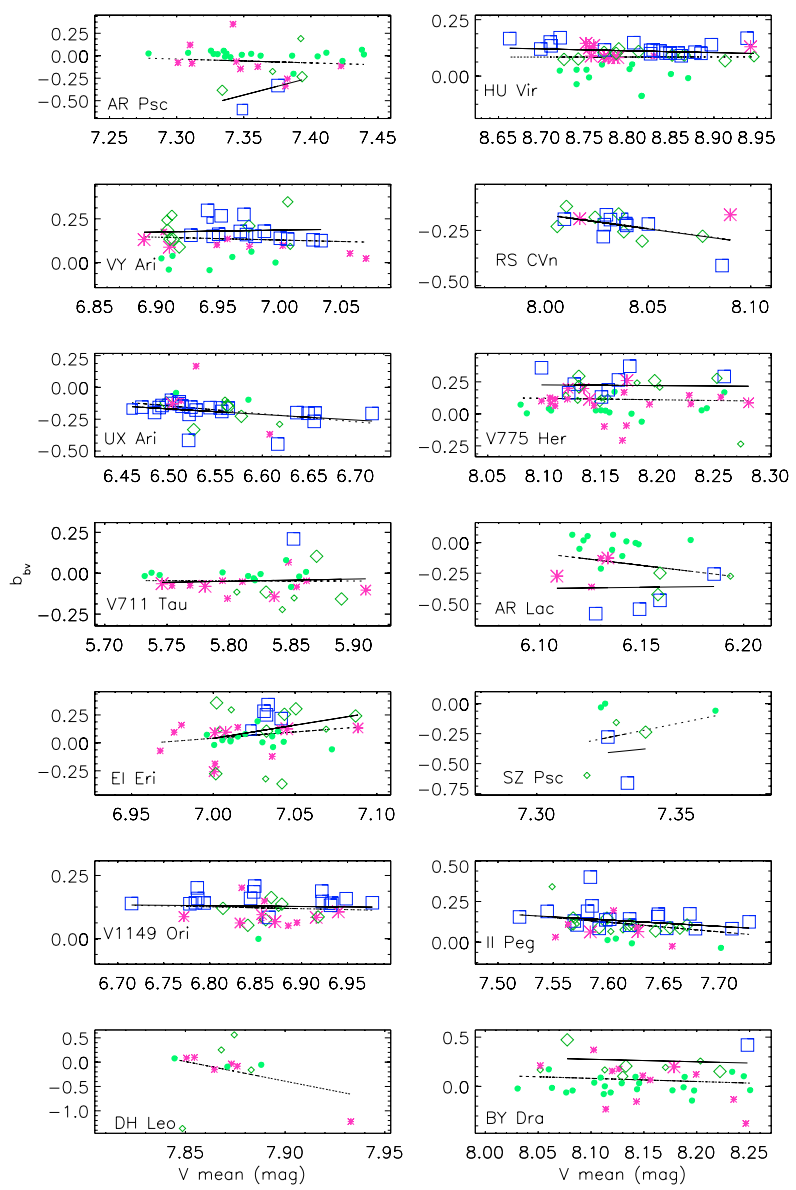

Fig. 10. Slope $b_{b v}$ vs. mean $V$ magnitude. Different symbols and sizes indicate different correlation coefficients and significance levels, respectively (see text). The solid and dotted lines are linear fits to significant values $(\alpha \leq 0.1)$ and to all data, respectively.

We take into consideration also the flux coming from the earliertype component and, whenever it is the case, from a tertiary component. The stellar fluxes were determined by using the NextGen atmosphere models of Hauschildt et al. (1999) for solar metallicity and convolved with the passbands of the $U B V$ system (Johnson 1953) as tabulated in Buser (1978) and Buser \& Kurucz (1978). The adopted values of the component stellar effective temperature, radius and gravity are listed in Sect. 2 . The mentioned stellar fluxes and physical parameters were used to compute the total flux ratios between the cool and hot components in the $U, B$ and $V$ bands which are listed in Table 1, as well as to compute the magnitude variations arising from proximity and reflection effects according to Eqs. (1) and (6) of Morris \& Naftilan (1993). We assume that only the component whose total flux dominates the system's luminosity is active. For instance, in the case of BY Dra, although both components have the same spectral class and should have similar levels of magnetic activity, we assume that only one component is active. In the case of RS CVn, the K0 IV component, although less luminous than the F5 IV component, will be considered the active one. Limbdarkening coefficients, different for the unperturbed and the spotted photosphere, are taken from Diaz-Cordoves et al. (1995). We have computed the model magnitude and color variation for a grid of values of temperature and covering fraction of spots and faculae. The covering fraction was varied from 0 to 0.50 with 0.01 increments, whereas the temperature of the surface inhomogeneities was varied from $3200 \mathrm{~K}$ up to $6800 \mathrm{~K}$ with $100 \mathrm{~K}$ increments. For instance, we have computed our modelling also for a range of gravity values $(\Delta \log g \pm 1.5)$ and effective temperature $\left(\Delta T_{\text {eff }} \pm 150\right)$ for sub-giant non-eclipsing binary stars in our sample, their values being poorly determined. In our modelling approach gravity-darkening effects are neglected by considering that these effects tend to cancel out when computing the flux difference between opposite hemispheres. As reported in Sect. 2, the reflection effect is negligible for all program stars, whereas the proximity effect is marginal for V711 Tau and AR Lac. However, we notice that such an effect does not play any significant role in the observed color variation as verified by using Eqs. (1) and (6) of Morris \& Naftilan (1993).

\subsection{Results}

In Fig. 11 for each program star we plot the observed $B-V$ (green filled bullets) and $U-B$ (blue open triangles) vs. $V$ magnitude variations and the model amplitudes (green small dots for $B-V$ and red small crosses for $U-B$ ) corresponding to all possible combinations of spot temperature and area. Specifically, for a given spot temperature the model solutions for different values of the filling factor lie along a dotted-like curve. Dotted curves of different slopes correspond to different spot temperatures. As expected, model solutions are not unique: different combinations of temperature and filling factor can determine the same variation amplitudes. In general we found that the warmer the spots, the larger their filling factor must be to fit the observed amplitude variations. Since our model is not able to obtain unique solutions, we will not focus on the found ranges of temperature and filling factor values, rather we shall discuss the possibility of fitting the observed blueing by considering the contribution of earlier-type components. Here we present the results of the modelling for the individual program stars.

AR Psc: color variations are correlated $(\alpha<0.1)$ to $V$ mag variations in $11 \%$ of light curves. The model reveals that the observed blueing can be accounted for in two out of three mean epochs, and within the photometric accuracy, by the presence of the earlier-type $\mathrm{G5} / 6 \mathrm{~V}$ component. In other words, the observed magnitude and color variations in two out of three cases fall within the area of model solutions.

VY Ari: color variations are correlated $(\alpha<0.1)$ to $V$ mag variations in $63 \%$ of light curves. Consistent with its classification as spot-dominated star, the observed reddening can be attributed to the presence of spots.

UX Ari: color variations are correlated $(\alpha<0.1)$ to $V$ mag variations in $83 \%$ of light curves. The model reveals that the earliertype G5 V component cannot account for the observed blueing. V711 Tau: color variations are correlated $(\alpha<0.1)$ to $V$ mag variations in $29 \%$ of light curves. The model shows that the earlier-type G5 V component can account for the observed blueing (except for one mean epoch).

EI Eri: color variations are correlated $(\alpha<0.1)$ to $V$ mag variations in $39 \%$ of light curves. Consistent with its classification as spot-dominated star, the observed reddening can be attributed to the presence of spots.

V1149 Ori: color variations are correlated $(\alpha<0.1)$ to $V$ mag variations in $77 \%$ of light curves. Notwithstanding the presence of the F8 $\mathrm{V}$ component, this star does not show any evidence of blueing. Consistent with its classification as a spot-dominated star, its color variations can be attributed to the presence of spots. DH Leo: since no color variations are found to be correlated ( $\alpha<0.10)$ to $V$ mag variations, we could not make a comparison with model solutions. 


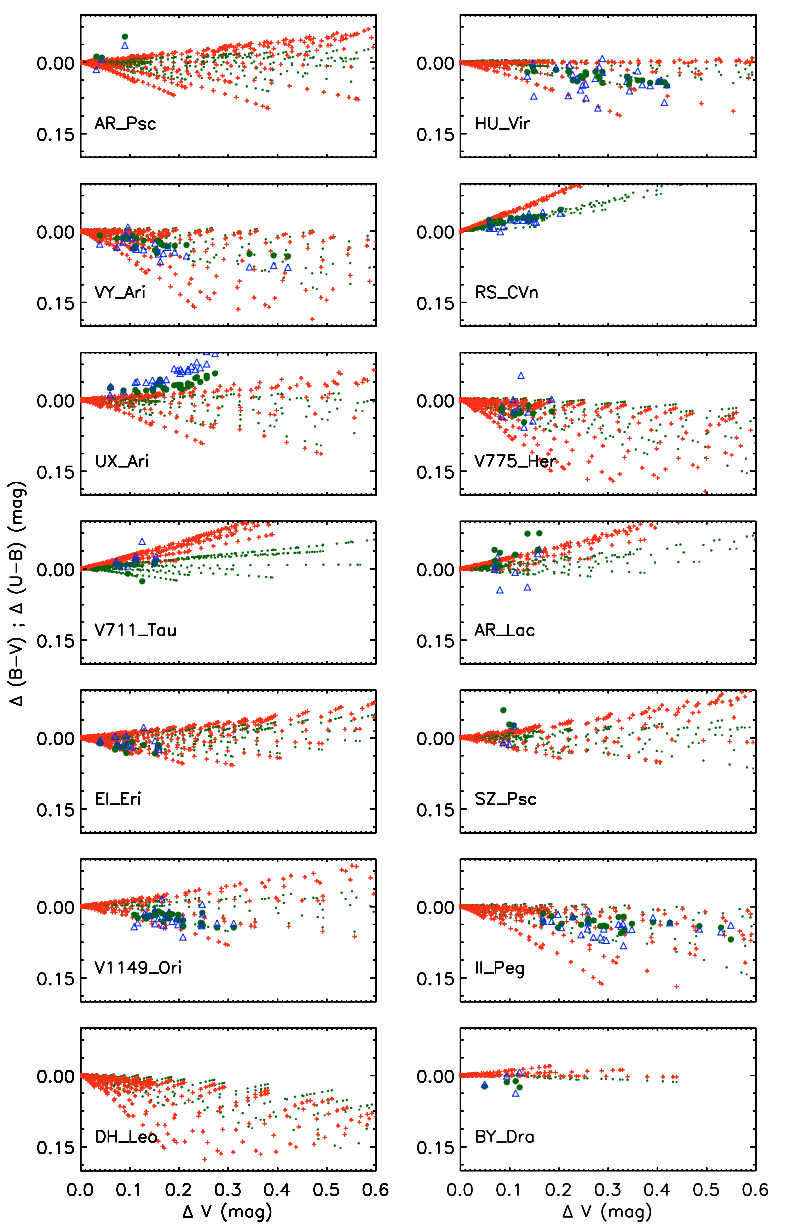

Fig. 11. Results of our model. Green filled bullets $(B-V)$ and blue open triangles $(U-B)$ represent the observed magnitude and color variations. Green small dots $(B-V)$ and red small crosses $(U-B)$ represent the family of model solutions.

HU Vir: color variations are correlated $(\alpha<0.1)$ to $V$ mag variations in $73 \%$ of light curves. Consistent with its classification as spot-dominated star, the observed reddening can be attributed to the presence of spots.

RS CVn: color variations are correlated $(\alpha<0.1)$ to $V$ mag variations in $95 \%$ of light curves. The model shows that the flux contribution by the earlier-type F5 V component can account only for the $B-V$ blueing, whereas the $U-B$ color variations are systematically smaller than the model variations.

V775 Her: color variations are correlated $(\alpha<0.1)$ to $V$ mag variations in $38 \%$ of light curves. Consistent with its classification as spot-dominated star, the observed reddening can be attributed to the presence of spots.

AR Lac: color variations are correlated $(\alpha<0.1)$ to $V$ mag variations in $39 \%$ of light curves. The model, with flux contribution by the earlier-type G2 IV component and spots, fits neither the $B-V$ blueing nor the $U-B$ reddening. Different to what is expected in the case of spots, the $B-V$ variations are systematically larger than the $U-B$ variations.

SZ Psc: color variations are correlated $(\alpha<0.1)$ to $V$ mag variations in $37 \%$ of light curves. The model reveals that the earliertype component cannot account for the observed blueing.

II Peg: color variations are correlated $(\alpha<0.1)$ to $V$ mag variations in $69 \%$ of light curves. Consistent with its classification as a spot-dominated star, the observed reddening can be attributed to the presence of spots.
BY Dra: color variations are correlated $(\alpha<0.1)$ to $V$ mag variations in $22 \%$ of light curves. Our model cannot fit the observed color variations, likely depending on the assuming that only one component is active and contributing to the observed color variations.

We note that among blueing stars, the presence of an earlier-type component can explain the observed blueing only in the case of V711 Tau. As expected for all the reddening stars, the spot model can fit the observed color variations in all mean epochs.

\section{Discussion}

The major result inferred from the analysis presented in Sect. 4 is the existence of reddening and blueing stars which can be either color-correlated or color-uncorrelated. As already anticipated, we guess that the existence of different patterns of color variation and different degrees of color-magnitude correlation can mainly arise from two circumstances: 1) the presence of a fainter component of the binary system whose activity level is not negligible; 2) the presence of hot faculae either spatially and temporally not correlated to cool spots. The latter represent, in turn, strong observational evidence in favour of the existence of faculae at least in some of our program stars.

Let us discuss the first circumstance. The activity level primarily depends on rotation rate and depth of the convection zone (see, e.g., Messina et al. 2001, 2003). Specifically, stars with a shorter rotation period and deeper convection zone show photometric variability larger than slower-rotating and earlier spectraltype stars. For example, the activity level of the G5 V component of UX Ari is much smaller than that of the K0 IV component, because of the smaller depth of the convection zone, although both components have the same rotation period. The luminosity difference between these components makes even more negligible the contribution by the G5 V component to the observed system's variability. In order to quantify this contribution by the earlier-type component, we have taken from the work of Messina et al. (2001) the maximum light curve amplitude expected in the $V$ band for the fainter active component of each program star. Using the $\left\langle b_{b v}\right\rangle$ and $\left\langle b_{u b}\right\rangle$ values from Table 6, although these values refer to the whole system, we have computed the approximate amplitude expected also in the $B$ and $U$ bands. Finally, considering the luminosity ratio between the cool and hot components as listed in Table 1, we have computed the maximum amplitude of the magnitude and color variations that could be attributed to the less active and fainter component. We found that the SB1 stars II Peg, VY Ari, HU Vir, as well the SB2 stars V1149 Ori, RS CVn, whose earlier-type companion is inactive (spectral type earlier than F5 V), or UX Ari, whose earlier-type companion is found with a negligible activity level, are all color-correlated stars. In these systems the variability arises from only one component and the color-magnitude variation remains correlated. On the other hand, we found that for V711 Tau, EI Eri, AR Lac, DH Leo and BY Dra the variations arising from the fainter companion are significant, of the order of a few percent of magnitude. All these stars are color-uncorrelated (the color-magnitude correlation is lowest for BY Dra, whose components have similar activity levels). We suggest that since the surface inhomogeneities are distributed differently on both components, the respective patterns of color variations are not coherent and the correlation is more frequently lost. AR Psc, V775 Her and SZ Psc are the exceptions being color-uncorrelated, although the variability contribution by the fainter companion is found to be negligible. The existence of faculae may play a major role in preventing the correlation in 
these stars. Indeed, AR Psc and SZ Psc were found to have negative $b_{u b v}$ and with a very low significance level, respectively.

Let us discuss the second circumstance. If we consider colorcorrelated stars, we find that the slopes of the colors vs. the $V$ mag variations are not constant over time. Since the slope value mostly depends on the average temperature of inhomogeneities, we deduce that it varies with time. Such variation may arise from the simultaneous presence of inhomogeneities of different temperatures, e.g., cool spots and hot faculae, whose relative area and flux contributions are variable.

The existence of hot faculae in active stars is documented in a number of works. Light curve inversion methods, widely used to extract information on the properties of stellar active regions, have generally assumed that dark spots are the dominant magnetic feature responsible for the observed brightness variations. Indeed, it has been generally found that for the most active stars, i.e. stars with an activity level much higher than that of the Sun, neither faculae nor network elements are required to obtain satisfactory $V$-band light curve models, the effect of starspots being dominant (e.g. Henry et al. 1995a; Lanza et al. 1998). Since the 1990s, some authors began to realize that, for less active stars, the variability at optical wavelengths is significantly influenced, if not dominated, by bright faculae. Radick et al. (1990, 1998) found that the optical brightness of stars of solar age, or older, increases with increasing chromospheric activity level over time scales of several years, suggesting that such brightness enhancement may be mostly attributed to bright features. Radick et al. proposed a scenario according to which young and rapidly rotating stars arrange their surface magnetic flux predominantly into dark spots, whereas, when stars age and their rotation slows down, bright facula-like structures are favoured. However, the rotational modulation of the optical flux remains dominated by dark spots in both young and old stars.

In a pilot program, Mirtorabi et al. (2003) investigated the correlation between the optical light curve and the $\mathrm{TiO}$ absorption strength for the evolved chromospherically active star $\lambda$ And, and found clear evidence that the $V$-band and near-IR continua light variation primarily arise from bright rather than dark starspots. O'Neal et al. (1998) found, from spectroscopic data, some evidence for multiple temperatures of the brightness inhomogeneities on II Peg. In the most active stars faculae seem to be necessary to account for their UV excess with respect to inactive stars (e.g. Amado 2003). The blueing of three stars in our sample, UX Ari, V711 Tau and RS CVn, has been previously investigated by Aarum Ulvas \& Engvold (2003) and Aarum Ulvas \& Henry (2005), and attributed to the presence of faculae.

We hypothesize that there are epochs when spots and faculae are spatially associated so that they can produce correlated magnitude and color variations. Although the area and/or temperature ratio can change, the correlation is preserved. There are other epochs in which spots and faculae are mostly spatially and temporally uncorrelated, e.g. spots and faculae have lifetimes that are significantly different and, when new spots or faculae emerge in the photosphere, their phase coherence with older patterns is lost. Although the global activity pattern producing the $V$-band modulation is stable, that producing the color variation is less stable over the same timescale. In other words, during these epochs faculae seem to act as an interference source which destroys the correlation between color and magnitude variations arising from spots only.

In our sample the reddening stars EI Eri, V775 Her and DH Leo and the blueing stars SZ Psc and AR Psc, whose fainter components were shown to give no contribution to the observed variability, are the best candidates for hosting faculae which are most of the time uncorrelated with spots. Although the correlation between spots and faculae is absent on the rotational time scale, it is still present on the longer time scale. As shown in the bottom panels of Figs. 2 and 7, as these stars approach the maximum activity level and the total spottedness increases (making the star fainter), the total amount of faculae increases (making the star bluer). However, the slope and the correlation coefficient are much smaller than in color-correlated stars.

The remaining blueing stars AR Lac, RS CVn, and UX Ari are the best candidates to host faculae, since their earlier-type stellar component cannot account for the observed blueing, according to the results of our model.

\section{Conclusions}

The long-term monitoring project of active close binary systems carried out at OAC has allowed us to collect a time-extended database of multiband high-precision photometric observations for a sample of 14 program stars. Correlation, and regression analyses, as well as simple modelling with these data has allowed us to discover and interpret the existence of color vs. magnitude variations showing different patterns and levels of correlation. Here we report the most relevant conclusions of our study:

\section{General conclusions:}

- Active close binary systems show magnitude and color variations. Such variations are correlated over a few seasons, but the correlation is lost in other seasons. The correlation is found more frequently (in more that $60 \%$ of the observed seasons) in single- and double-lined stars in which only one component is active, the other component being inactive (F spectral type or earlier) or with a negligible activity level. The correlation is much less frequent (in less that $40 \%$ of observed seasons) in double-lined stars whose fainter component has a non-negligible level of activity. In these stars the correlation may be prevented by different spot distributions in the two active components.

- The slope of the relation between magnitude and color variations is found to vary from season to season in all stars. Such variation may arise from a variable flux contribution by simultaneous inhomogeneities of different temperature, such as cool spots and hot faculae, which make the average temperature variable and, therefore, the computed relation slope.

- In single- and double-lined stars in which only one component is active the correlation between magnitude and color variation is found in a few seasons to be absent, likely because spots and faculae are spatially and temporally uncorrelated. In such cases faculae act as a noise source which makes the observed color pattern highly unstable. In doublelined stars where the fainter component has a significant level of activity, beside faculae, a different inhomogeneity pattern on the fainter component can prevent the correlation between magnitude and color variation.

- Our modelling shows that for blueing stars an earlier-type component can provide a significant contribution to the observed blueing. However, it alone cannot account for the variable slope of the magnitude-color relations.

Specific conclusions:

II Peg, VY Ari, HU Vir and V1149 Ori are reddening and color-correlated stars whose variability originates only from the primary component and is dominated by cool spots. However, faculae can be present and alter the reddening 
slope, but preserving in most seasons their correlation with spots;

V775 Her is a reddening and color-uncorrelated star whose variability originates only from the primary component and it arises from either spots or facular activity not correlated to spots;

BY Dra, EI Eri and DH Leo are reddening and coloruncorrelated stars whose variability originates from both components and is dominated by cool spots. The correlation between their magnitude and color variations is frequently lost due to either a non-negligible activity level in the fainter companion or the presence of faculae non-correlated to spots;

UX Ari and RS CVn are blueing and color-correlated stars whose activity takes place only in the primary component. Their short-term color variations are dominated by faculae, which are most of the time correlated to spots. The long-term color variation can partly arise from the hotter companion;

AR Psc and SZ Psc are blueing and color-uncorrelated stars whose activity takes place only in the primary component and whose color variations are dominated by faculae which are on the rotation timescale rarely correlated to spot activity; AR Lac and V711 Tau are blueing and color-uncorrelated stars whose activity takes place in both components and whose color variations are dominated by faculae. These are rarely correlated to magnitude variation due to either non-correlated spot/faculae activity on the rotation timescale and a non-negligible activity level in the fainter companion. V711 Tau is the only star in our sample whose blueing, but not the slope variations, could be entirely attributed to the flux contribution by the earlier-type component.

Acknowledgements. The acquisition of photometric data over so many years with the Catania APT has been possible thanks to the dedicated and highly competent technical assistance of a number of people, notably P. Bruno, E. Martinetti and S. Sardone. Active star research at the INAF-Catania Astrophysical Observatory is funded by MUR (Ministero dell'Università e della Ricerca), and the Regione Sicilia, whose financial support is gratefully acknowledged. The extensive use of the SIMBAD and ADS databases, operated by the CDS center (Strasbourg, France), is also gratefully acknowledged. I would like to thank Dr. A. F. Lanza for his valuable comments and useful discussion, Dr. G. Cutispoto for his valuable support in this project, and the Referee, Dr. Panos G. Niarchos, for careful reading of the manuscript.

\section{References}

Aarum Ulvas, V., \& Engvold, O. 2003, A\&A, 399, L11

Aarum Ulvas, V., \& Henry, G. W. 2005, AN, 326, 292

Amado, P. J. 2003, A\&A, 404, 631

Baliunas, S. L., Boyd, L. J., Genet, R. M., Hall, D. S., \& Criswell, S. 1985, IAPPP Comm., 22, 47

Barden, S. C. 1984 , AJ, 89, 683

Bevington, P. R. 1969, in Data Reduction and Error Analysis for the Physical Sciences (New York: McGraw-Hill Book Company)

Bolton, C. T., Aslan, Z., Kamper, K. W., \& Lyons, R. W. 1981, AJ, 86, 1267

Bopp, B. W., \& Evans, D. S. 1973, MNRAS, 164, 343

Bopp, B. W., Saar, S. H., Ambruster, C., et al. 1989, ApJ, 339, 1059

Boyd, L. J., Genet, R. M., \& Hall, D. S. 1984, IAPPP Comm., 15, 20

Buser, R. 1978, A\&A, 62, 411

Buser, R., \& Kurucz, R. L. 1978, A\&A, 70, 555

Byrne, P. B., \& Rodonò, M. 1983, in Activity in Red Dwarf Stars, ed. P. B. Byrne, \& M. Rodonò (Reidel, Dordrecht), IAU Coll., 71

Carlos, R. C., \& Popper, D. M. 1971, PASP, 83, 504

Catalano, S., \& Rodonò, M. 1967, Mem. Soc. Astron. It., 38, 395

Catalano, S., \& Rodonò, M. 1974, PASP, 86, 390

Chisari, D., \& Lacona, G. 1965, Mem. Soc. Astron. It., 36, 433

Cristaldi, S., \& Rodonò, M. 1968, IBVS, 252

Cristaldi, S., Narbone, M., \& Rodonò, M. 1968, IBVS, 317

Cox, A. N. 2000, in Allen's Astrophysical Quantities, 4th Ed. (Springer, AIP Press)
Cutispoto, G. 1990, A\&AS, 84, 397 Cutispoto, G. 1991, A\&AS, 89, 435 Cutispoto, G. 1992, A\&AS, 95, 397

Cutispoto, G. 1993, A\&AS, 102, 655 Cutispoto, G. 1995, A\&AS, 111, 507 Cutispoto, G. 1998, A\&AS, 131, 321

Cutispoto, G., Messina, S., \& Rodonò, M. 2001, A\&A, 367, 910 Cutispoto, G., Messina, S., \& Rodonò, M. 2003, A\&A, 400, 659

Dorren, J. D. 1987, ApJ, 320, 756

Diaz-Cordoves, J., Claret, A., \& Gimenez, A. 1995, A\&AS, 110, 329

Duemmler, R., \& Aarum, V. 2001, A\&A, 370, 974

Eaton, J. A., \& Henry, G. W. 2007, PASP, 119, 259

Fekel, F. C. 1983, ApJ, 268, 274

Fekel, F. C. 1996, AJ, 112, 269

Fekel, F. C., \& Henry, G. W. 2005, AJ, 129, 1669

Fekel, F. C., Quigley, R., Gillies, K., \& Africano, J. L. 1987, AJ, 94, 726

Frasca, A., Biazzo, K., Catalano, S., et al. 2005, A\&A, 432, 647

Gershberg, R. E., \& Shakhovskaya, N. I. 2003, Astron. Astrophys. Trans., 22, 441

Jetsu, L., Pagano, I., Moss, D., et al. 1997, A\&A, 326, 698

Johnson, H. L., \& Morgan, W. W. 1953, ApJ, 117, 313

Hall, D. S. 1976, in Multiple Period Variable Stars, ed. W. S. Fitch, IAU Coll., 29, 287

Hauschildt, P. H., Allard, F., \& Baron, E. 1999, ApJ, 512, 377

Henry, G. W., Eaton, J. A., Hamer, J., \& Hall, D. S. 1995a, ApJS, 97, 513

Henry, G. W., Fekel, F. C., \& Hall, D. S. 1995b, AJ, 110, 2926

Hongguam, S., Xuefu, L., \& Shenghong, G. 2006, New Astron., 11, 287

Kron, G. E. 1950, AJ, 55, 69

Imbert, M. 1979, A\&AS, 38, 401

Lanza, A. F., Rodonò, M., \& Zappalà, R. 1993, A\&A, 269, 351

Lanza, A. F., Rodonò, M., \& Zappalà, R. 1994, A\&A, 290, 861

Lanza, A. F., Catalano, S., Cutispoto, G., Pagano, I., \& Rodonò, M. 1998, A\&A, 332,541

Lanza, A. F., Rodonò, M., Mazzola, L., \& Messina, S. 2001, A\&A, 376, 1011

Lanza, A. F., Piluso, N., Rodonò, M., Messina S., \& Cutispoto, G. 2006, A\&A, 455,606

Messina, S. 1998, Ph.D. Thesis, Univ. of Catania, Italy

Messina, S., \& Guinan, E. F. 2002, A\&A, 393, 225

Messina, S., \& Guinan, E. F. 2003, A\&A, 409, 1017

Messina, S., Rodonò, M., \& Guinan, E. F. 2001, A\&A, 366, 215

Messina, S., Pizzolato, N., Guinan, E. F., \& Rodonò, M. 2003, A\&A, 410, 671

Messina, S., Rodonò, M., \& Cutispoto, G. 2004, AN, 325, 660

Mirtorabi, M. T., Wasatonic, R., \& Guinan, E. F. 2003, AJ, 125, 3265

Mohin, S., \& Raveendran, A. V. 1989, JApA, 5, 159

Morris, L. S., \& Naftilan, S. A. 1993, ApJ, 419, 344

Oliver, J. P. 1974, Ph.D. Thesis, University of California, Los Angeles (CA, USA)

O’Neal, D., Saar, S. H., \& Neff, J. E. 1998, ApJ, 501, L73

Radick, R. R., Lockwood, G. W., \& Baliunas, S. L. 1990, Science, 247, 39

Radick, R. R., Lockwood, G. W., Skiff, B. A., \& Baliunas, S. L. 1998, ApJS, 118,239

Reglero, V., Gimenez, A., \& Estela, A. 1990, A\&A, 231, 375

Rodonò, M. 1965, Ph.D. Thesis, University of Catania, Italy

Rodonò, M. 1992, in Surface Inhomogeneities on Late-Type Stars, ed. P. B.

Byrne, \& D. J. Mullan (Heidelberg, Germany: Springer-Verlag), Lecture Notes in Physics, 397, 201

Rodonò, M., \& Cutispoto, G. 1992, A\&AS, 95, 55

Rodonò, M., \& Cutispoto, G. 1994a, Mem. Soc. Astron. It., 65, 83

Rodonò, M., \& Cutispoto, G. 1994b, in Cool Stars, Stellar Systems, and the Sun, Eighth Cambridge Workshop, ed. J.-P. Caillault (San Francisco), ASP Conf. Ser., 64, 459

Rodonò, M., Pazzani, V., \& Cutispoto, G. 1983, in Activity in Red-Dwarf Stars, ed. P. B. Byrne, \& M. Rodonò (Reidel, Dordrecht), IAU Coll., 71, 179

Rodonò, M., Lanza, A. F., \& Catalano, S. 1995, A\&A, 301, 75

Rodonò, M., Messina, S., Lanza, A. F., Cutispoto, G., \& Teriaca, L. 2000, A\&A, 358,624

Rodonò, M., Lanza, A. F., \& Becciani, U. 2001a, A\&A, 371, 174

Rodonò, M., Cutispoto, G., Lanza, A. F., \& Messina, S. 2001b, AN, 322, 333

Rucinski, S. M. 1977, PASP, 89, 280

Strassmeier, K. G. 1994, A\&A, 281, 395

Strassmeier, K. G., \& Bopp, B. W. 1992, A\&A, 259, 183

Strassmeier, K. G., Hall, D. S., Fekel, F. C., \& Scheck, M. 1993, A\&AS, 72, 291

Strassmeier, K. G., Bartus, J., Cutispoto, G., \& Rodonò, M. 1997, A\&AS, 125, 11

Wacker, S. W., Guinan, E. F., McCook, G. P., Lochner, J. P., \& Paczkowski, B. G. 1986, IBVS, 2920

Zeilik, M., Elston, R., Henson, G., \& Smith, P. 1982, IBVS, 2168 
Table 4. Summary of photometric observations: mean epoch of observation, mean, initial and final heliocentric Julian day, number of points in the light curve, brightest $V$ magnitude and light curve peak-to-peak amplitude in the $V$ band, $B-V$ and $U-B$ colors, standard deviation $\left(\sigma_{v}\right)$ of $v-c$ and $\left(\sigma_{c k 1-c}\right)$ of $c k 1-c$ differential observations. The telescopes are labelled with "A": Phoenix-25, "B": APT80/1, "C": ESO $50 \mathrm{~cm}$.

\begin{tabular}{|c|c|c|c|c|c|c|c|c|c|c|c|}
\hline Mean epoch & $\mathrm{HJD}_{\text {mean }}$ & $\mathrm{HJD}_{\text {ini }}$ & HJD $_{\text {end }}$ & $N_{\mathrm{m}}$ & $V_{\min }$ & $\Delta V$ & $B-V$ & $U-B$ & $\sigma_{v}$ & $\sigma_{c k 1-c}$ & Note \\
\hline \multicolumn{12}{|c|}{ AR Psc } \\
\hline 1987.93 & 7136.63 & 7129.64 & 7143.61 & 13 & 7.243 & 0.072 & 0.855 & 0.392 & 0.026 & 0.007 & $\mathrm{C}$ \\
\hline 1989.96 & 7879.09 & 7870.61 & 7887.57 & 13 & 7.273 & 0.056 & 0.856 & 0.380 & 0.022 & - & $\mathrm{C}$ \\
\hline 1990.83 & 8195.35 & 8178.90 & 8211.80 & 14 & 7.299 & 0.091 & 0.845 & 0.393 & 0.033 & 0.007 & A \\
\hline 1991.82 & 8556.83 & 8540.87 & 8572.78 & 11 & 7.343 & 0.065 & 0.837 & - & 0.021 & 0.006 & A \\
\hline 1992.00 & 8621.69 & 8589.79 & 8653.60 & 11 & 7.302 & 0.107 & 0.843 & 0.387 & 0.039 & 0.006 & A \\
\hline 1992.85 & 8934.10 & 8899.83 & 8968.37 & 20 & 7.312 & 0.056 & 0.840 & 0.398 & 0.018 & 0.005 & $A+B$ \\
\hline 1993.02 & 8994.98 & 8978.36 & 9011.61 & 17 & 7.290 & 0.094 & 0.856 & 0.388 & 0.031 & 0.005 & B \\
\hline 1993.87 & 9307.52 & 9247.81 & 9367.22 & 28 & 7.286 & 0.089 & 0.849 & 0.390 & 0.029 & 0.005 & $A+B$ \\
\hline 1994.66 & 9593.04 & 9577.58 & 9608.51 & 10 & 7.362 & 0.038 & 0.854 & 0.370 & 0.011 & 0.009 & B \\
\hline 1994.89 & 9679.41 & 9658.51 & 9700.31 & 11 & 7.340 & 0.063 & 0.844 & 0.385 & 0.020 & 0.006 & A \\
\hline 1995.49 & 9898.43 & 9887.96 & 9908.91 & 9 & 7.321 & 0.042 & 0.842 & 0.399 & 0.013 & 0.007 & $A+B$ \\
\hline 1995.76 & 9995.80 & 9981.84 & 10009.75 & 21 & 7.318 & 0.032 & 0.839 & 0.395 & 0.010 & 0.007 & $A+B$ \\
\hline 1995.85 & 10029.09 & 10011.50 & 10046.68 & 14 & 7.308 & 0.054 & 0.836 & 0.390 & 0.019 & 0.014 & B \\
\hline 1996.83 & 10388.10 & 10374.52 & 10401.67 & 10 & 7.304 & 0.090 & 0.833 & 0.364 & 0.029 & 0.003 & $A+B$ \\
\hline 1996.94 & 10429.04 & 10417.44 & 10440.65 & 17 & 7.308 & 0.039 & 0.834 & 0.382 & 0.012 & 0.005 & A \\
\hline 1997.06 & 10472.61 & 10450.63 & 10494.58 & 14 & 7.282 & 0.056 & 0.828 & 0.385 & 0.017 & 0.005 & A \\
\hline 1997.76 & 10728.10 & 10702.44 & 10753.75 & 22 & 7.269 & 0.085 & 0.828 & 0.386 & 0.026 & 0.006 & $A+B$ \\
\hline 1997.87 & 10768.21 & 10754.74 & 10781.68 & 16 & 7.275 & 0.067 & 0.827 & 0.392 & 0.019 & 0.006 & A \\
\hline 1997.98 & 10806.15 & 10782.69 & 10829.61 & 29 & 7.306 & 0.039 & 0.826 & 0.389 & 0.009 & 0.006 & $A+B$ \\
\hline 1998.08 & 10844.10 & 10832.61 & 10855.60 & 16 & 7.322 & 0.051 & 0.829 & 0.386 & 0.015 & 0.006 & A \\
\hline 1998.65 & 11051.50 & 11039.51 & 11063.48 & 8 & 7.324 & 0.077 & 0.835 & 0.381 & 0.028 & 0.006 & B \\
\hline 1998.87 & 11133.74 & 11078.85 & 11188.62 & 63 & 7.325 & 0.122 & 0.825 & 0.386 & 0.035 & 0.006 & $A+B$ \\
\hline 1999.08 & 11209.11 & 11192.62 & 11225.60 & 15 & 7.360 & 0.065 & 0.832 & 0.372 & 0.020 & 0.006 & A \\
\hline 1999.82 & 11480.11 & 11445.47 & 11514.74 & 26 & 7.370 & 0.025 & 0.827 & 0.391 & 0.007 & 0.007 & $A+B$ \\
\hline 1999.96 & 11529.70 & 11519.72 & 11539.68 & 11 & 7.372 & 0.030 & 0.822 & 0.389 & 0.010 & 0.007 & A \\
\hline 2000.06 & 11567.14 & 11545.68 & 11588.60 & 19 & 7.372 & 0.043 & 0.829 & 0.382 & 0.015 & 0.007 & A \\
\hline 2000.68 & 11792.51 & 11757.57 & 11827.45 & 12 & 7.331 & 0.059 & 0.872 & 0.395 & 0.020 & 0.006 & B \\
\hline 2000.97 & 11901.50 & 11852.41 & 11950.59 & 24 & 7.312 & 0.186 & 0.822 & 0.374 & 0.064 & 0.006 & $A+B$ \\
\hline 2001.81 & 12206.99 & 12166.61 & 12247.37 & 22 & 7.269 & 0.134 & 0.841 & 0.384 & 0.034 & 0.006 & $A+B$ \\
\hline 2002.02 & 12283.67 & 12253.75 & 12313.59 & 12 & 7.364 & 0.095 & 0.822 & 0.370 & 0.030 & 0.006 & A \\
\hline 2002.77 & 12555.17 & 12521.57 & 12588.76 & 15 & 7.399 & 0.047 & 0.833 & 0.370 & 0.019 & 0.008 & $A+B$ \\
\hline 2003.00 & 12639.67 & 12612.72 & 12666.61 & 15 & 7.392 & 0.063 & 0.827 & 0.368 & 0.016 & 0.008 & A \\
\hline 2003.75 & 12915.43 & 12896.97 & 12933.90 & 17 & 7.402 & 0.075 & 0.832 & 0.372 & 0.019 & 0.006 & A \\
\hline 2003.90 & 12968.25 & 12934.82 & 13001.68 & 26 & 7.407 & 0.062 & 0.834 & 0.373 & 0.017 & 0.012 & A \\
\hline 2004.90 & 13336.72 & 13309.78 & 13363.65 & 19 & 7.269 & 0.159 & 0.827 & 0.375 & 0.059 & 0.006 & A \\
\hline \multicolumn{12}{|c|}{ VY Ari } \\
\hline 1991.79 & 8545.90 & 8531.93 & 8559.87 & 19 & 6.827 & 0.183 & 0.977 & 0.573 & 0.061 & 一 & A \\
\hline 1991.94 & 8599.28 & 8566.88 & 8631.68 & 16 & 6.855 & 0.182 & 0.986 & - & 0.051 & - & A \\
\hline 1992.11 & 8662.13 & 8636.66 & 8687.60 & 20 & 6.901 & 0.149 & 0.980 & 0.658 & 0.048 & - & A \\
\hline 1992.81 & 8919.86 & 8897.91 & 8941.80 & 28 & 6.884 & 0.168 & 0.983 & 0.666 & 0.050 & - & A \\
\hline 1992.92 & 8961.29 & 8943.79 & 8978.78 & 17 & 6.899 & 0.162 & 0.982 & 0.655 & 0.050 & - & A \\
\hline 1993.08 & 9016.64 & 8988.66 & 9044.62 & 16 & 6.930 & 0.090 & 0.983 & 0.653 & 0.027 & - & A \\
\hline 1993.71 & 9246.92 & 9233.97 & 9259.87 & 20 & 6.939 & 0.127 & 0.991 & 0.664 & 0.042 & - & A \\
\hline 1993.99 & 9350.58 & 9327.51 & 9373.65 & 23 & 6.899 & 0.177 & 0.976 & 0.649 & 0.059 & 0.013 & A \\
\hline 1994.12 & 9398.62 & 9374.65 & 9422.59 & 23 & 6.899 & 0.215 & 0.975 & 0.673 & 0.073 & 0.013 & $A+B$ \\
\hline 1994.83 & 9655.53 & 9607.56 & 9703.49 & 28 & 6.909 & 0.128 & 0.981 & 0.624 & 0.040 & 0.011 & $A+B$ \\
\hline 1995.07 & 9745.90 & 9715.50 & 9776.30 & 13 & 6.915 & 0.112 & 0.953 & 0.617 & 0.037 & 0.011 & $A+B$ \\
\hline 1995.74 & 9986.73 & 9972.57 & 10000.88 & 17 & 6.857 & 0.113 & 0.979 & 0.657 & 0.034 & 0.014 & B \\
\hline 1995.82 & 10018.22 & 10002.64 & 10033.81 & 23 & 6.865 & 0.082 & 0.984 & 0.653 & 0.027 & 0.014 & $A+B$ \\
\hline 1995.93 & 10057.17 & 10035.63 & 10078.71 & 22 & 6.866 & 0.093 & 0.989 & 0.646 & 0.033 & 0.014 & $A+B$ \\
\hline 1996.07 & 10110.13 & 10088.68 & 10131.59 & 26 & 6.889 & 0.039 & 0.976 & 0.652 & 0.012 & 0.014 & A \\
\hline 1996.90 & 10413.44 & 10388.42 & 10438.45 & 16 & 6.878 & 0.167 & 0.973 & 0.617 & 0.058 & 0.015 & B \\
\hline 1997.07 & 10475.84 & 10453.43 & 10498.25 & 18 & 6.864 & 0.155 & 0.977 & 0.609 & 0.053 & 0.015 & B \\
\hline 1997.84 & 10755.96 & 10703.47 & 10808.46 & 14 & 6.913 & 0.089 & 0.953 & 0.607 & 0.023 & 0.016 & B \\
\hline 1998.07 & 10839.33 & 10817.42 & 10861.25 & 18 & 6.879 & 0.128 & 0.956 & 0.609 & 0.039 & 0.016 & B \\
\hline 1998.16 & 10873.26 & 10864.25 & 10882.27 & 7 & 6.903 & 0.081 & 0.965 & 0.607 & 0.029 & 0.016 & B \\
\hline 1998.70 & 11070.10 & 11039.56 & 11100.64 & 21 & 6.953 & 0.107 & 0.973 & 0.607 & 0.032 & 0.020 & B \\
\hline 1998.90 & 11144.97 & 11122.46 & 11167.48 & 10 & 6.952 & 0.210 & 0.984 & 0.623 & 0.069 & 0.020 & B \\
\hline 1999.04 & 11194.42 & 11176.45 & 11212.38 & 12 & 6.925 & 0.167 & 0.987 & 0.565 & 0.053 & 0.020 & B \\
\hline 1999.72 & 11443.15 & 11436.65 & 11449.65 & 4 & 6.924 & 0.145 & 1.002 & 0.607 & 0.068 & 0.014 & B \\
\hline 1999.93 & 11518.03 & 11506.57 & 11529.49 & 6 & 6.921 & 0.298 & 0.954 & 0.609 & 0.121 & 0.014 & B \\
\hline 2000.75 & 11817.97 & 11772.51 & 11863.42 & 57 & 6.823 & 0.421 & 0.987 & 0.687 & 0.147 & 0.007 & $A+B$ \\
\hline 2000.93 & 11885.70 & 11865.74 & 11905.66 & 27 & 6.831 & 0.392 & 0.987 & 0.677 & 0.143 & 0.007 & A \\
\hline
\end{tabular}


Table 4. continued.

\begin{tabular}{|c|c|c|c|c|c|c|c|c|c|c|c|}
\hline Mean epoch & $\mathrm{HJD}_{\text {mean }}$ & HJD $_{\text {ini }}$ & $\mathrm{HJD}_{\text {end }}$ & $N_{\mathrm{m}}$ & $V_{\min }$ & $\Delta V$ & $B-V$ & $U-B$ & $\sigma_{v}$ & $\sigma_{c k 1-c}$ & Note \\
\hline 2001.06 & 11934.13 & 11906.66 & 11961.59 & 34 & 6.829 & 0.343 & 0.980 & 0.674 & 0.123 & 0.007 & $A+B$ \\
\hline 2001.74 & 12181.79 & 12164.94 & 12198.63 & 31 & 6.855 & 0.191 & 0.991 & 0.674 & 0.054 & 0.005 & $A+B$ \\
\hline 2001.85 & 12220.23 & 12201.89 & 12238.57 & 25 & 6.871 & 0.156 & 0.987 & 0.663 & 0.053 & 0.005 & $A+B$ \\
\hline 2001.95 & 12256.25 & 12240.76 & 12271.74 & 19 & 6.877 & 0.151 & 0.982 & 0.665 & 0.040 & 0.005 & $A+B$ \\
\hline 2002.06 & 12296.66 & 12275.71 & 12317.61 & 19 & 6.941 & 0.072 & 0.982 & 0.678 & 0.022 & 0.005 & A \\
\hline 2002.77 & 12556.37 & 12537.92 & 12574.81 & 29 & 6.860 & 0.088 & 0.976 & 0.683 & 0.024 & 0.005 & A \\
\hline 2002.91 & 12608.74 & 12583.82 & 12633.66 & 29 & 6.880 & 0.096 & 0.971 & 0.670 & 0.030 & 0.005 & A \\
\hline 2003.03 & 12652.63 & 12634.65 & 12670.60 & 28 & 6.866 & 0.086 & 0.973 & 0.673 & 0.027 & 0.006 & A \\
\hline 2003.76 & 12917.35 & 12894.92 & 12939.79 & 60 & 6.858 & 0.105 & 0.975 & 0.671 & 0.028 & 0.005 & A \\
\hline 2003.91 & 12971.78 & 12947.80 & 12995.76 & 45 & 6.844 & 0.092 & 0.974 & 0.675 & 0.025 & 0.008 & A \\
\hline 2004.06 & 13026.63 & 12999.66 & 13053.60 & 16 & 6.880 & 0.060 & 0.966 & 0.676 & 0.020 & 0.008 & A \\
\hline 2004.73 & 13273.39 & 13255.86 & 13290.91 & 38 & 6.836 & 0.154 & 0.974 & 0.669 & 0.046 & 0.006 & A \\
\hline 2004.94 & 13348.71 & 13291.82 & 13405.60 & 86 & 6.832 & 0.158 & 1.076 & 0.768 & 0.047 & 0.006 & A \\
\hline \multicolumn{12}{|c|}{ UX Ari } \\
\hline 1990.04 & 7907.70 & 7893.72 & 7921.69 & 12 & 6.450 & 0.103 & 0.865 & 0.392 & 0.040 & 0.006 & A \\
\hline 1990.15 & 7948.64 & 7926.68 & 7970.60 & 12 & 6.474 & 0.078 & 0.867 & 0.375 & 0.024 & 0.006 & A \\
\hline 1990.81 & 8188.94 & 8168.98 & 8208.90 & 26 & 6.477 & 0.061 & 0.862 & 0.389 & 0.016 & 0.009 & A \\
\hline 1991.01 & 8262.26 & 8228.84 & 8295.68 & 17 & 6.509 & 0.040 & 0.855 & 0.374 & 0.013 & 0.009 & A \\
\hline 1991.82 & 8555.41 & 8529.95 & 8580.87 & 22 & 6.498 & 0.057 & 0.855 & 0.337 & 0.014 & 0.008 & A \\
\hline 1992.09 & 8655.67 & 8630.70 & 8680.64 & 17 & 6.491 & 0.060 & 0.852 & 0.379 & 0.017 & 0.008 & A \\
\hline 1992.78 & 8909.17 & 8878.46 & 8939.87 & 29 & 6.396 & 0.202 & 0.860 & 0.380 & 0.054 & 0.009 & $\mathrm{~A}+\mathrm{B}$ \\
\hline 1992.94 & 8964.78 & 8940.88 & 8988.68 & 26 & 6.416 & 0.174 & 0.860 & 0.371 & 0.063 & 0.009 & $A+B$ \\
\hline 1993.12 & 9033.53 & 9001.46 & 9065.60 & 19 & 6.402 & 0.218 & 0.854 & 3.087 & 0.071 & 0.009 & $A+B$ \\
\hline 1993.82 & 9285.90 & 9233.98 & 9337.82 & 53 & 6.408 & 0.206 & 0.856 & 0.382 & 0.061 & 0.009 & $\mathrm{~A}+\mathrm{B}$ \\
\hline 1994.09 & 9386.01 & 9353.40 & 9418.63 & 10 & 6.362 & 0.197 & 0.868 & 0.373 & 0.065 & 0.009 & $A+B$ \\
\hline 1994.83 & 9655.57 & 9607.63 & 9703.52 & 27 & 6.380 & 0.225 & 0.865 & 0.382 & 0.076 & 0.008 & $A+B$ \\
\hline 1995.13 & 9767.87 & 9737.45 & 9798.28 & 15 & 6.398 & 0.146 & 0.870 & 0.386 & 0.051 & 0.009 & $A+B$ \\
\hline 1995.80 & 10009.86 & 9981.88 & 10037.85 & 39 & 6.422 & 0.203 & 0.855 & 0.358 & 0.058 & 0.009 & A \\
\hline 1995.91 & 10049.55 & 10039.75 & 10059.35 & 13 & 6.444 & 0.155 & 0.855 & 0.356 & 0.039 & 0.009 & A \\
\hline 1996.07 & 10107.99 & 10074.39 & 10141.59 & 38 & 6.460 & 0.190 & 0.850 & 0.355 & 0.060 & 0.009 & A \\
\hline 1996.89 & 10410.05 & 10386.41 & 10433.69 & 31 & 6.435 & 0.218 & 0.851 & 0.370 & 0.068 & 0.007 & $A+B$ \\
\hline 1997.04 & 10463.40 & 10435.48 & 10491.32 & 36 & 6.401 & 0.256 & 0.858 & 0.380 & 0.078 & 0.007 & A \\
\hline 1997.15 & 10507.12 & 10494.63 & 10519.60 & 10 & 6.465 & 0.197 & 0.846 & 0.363 & 0.068 & 0.007 & A \\
\hline 1997.74 & 10720.63 & 10702.45 & 10738.81 & 28 & 6.368 & 0.233 & 0.861 & 0.389 & 0.067 & 0.007 & $A+B$ \\
\hline 1998.09 & 10848.64 & 10814.67 & 10882.60 & 49 & 6.368 & 0.243 & 0.856 & 0.390 & 0.078 & 0.007 & $A+B$ \\
\hline 1998.77 & 11096.23 & 11039.57 & 11152.89 & 74 & 6.430 & 0.168 & 0.860 & 0.498 & 0.049 & 0.007 & $A+B$ \\
\hline 1999.05 & 11198.60 & 11157.56 & 11239.63 & 53 & 6.430 & 0.147 & 0.863 & 0.376 & 0.045 & 0.006 & $A+B$ \\
\hline 1999.77 & 11461.93 & 11447.86 & 11476.00 & 27 & 6.501 & 0.111 & 0.849 & 0.362 & 0.034 & 0.006 & $A+B$ \\
\hline 1999.87 & 11497.24 & 11477.96 & 11516.52 & 34 & 6.505 & 0.115 & 0.850 & 0.360 & 0.031 & 0.006 & $A+B$ \\
\hline 2000.10 & 11580.69 & 11546.76 & 11614.61 & 37 & 6.497 & 0.133 & 0.848 & 0.358 & 0.036 & 0.009 & $A+B$ \\
\hline 2000.68 & 11795.09 & 11775.59 & 11814.59 & 15 & 6.489 & 0.142 & 0.858 & 0.357 & 0.041 & 0.009 & $A+B$ \\
\hline 2000.86 & 11858.31 & 11810.88 & 11905.74 & 95 & 6.534 & 0.087 & 0.843 & 0.348 & 0.025 & 0.009 & $A+B$ \\
\hline 2001.08 & 11939.69 & 11907.74 & 11971.64 & 40 & 6.544 & 0.082 & 0.850 & 0.341 & 0.019 & 0.007 & $A+B$ \\
\hline 2001.76 & 12185.76 & 12163.58 & 12207.94 & 22 & 6.591 & 0.034 & 0.847 & 0.314 & 0.011 & 0.007 & $\mathrm{~A}+\mathrm{B}$ \\
\hline 2001.90 & 12239.33 & 12210.91 & 12267.76 & 19 & 6.597 & 0.043 & 0.844 & 0.335 & 0.012 & 0.007 & $A+B$ \\
\hline 2002.04 & 12291.72 & 12268.77 & 12314.66 & 15 & 6.586 & 0.061 & 0.844 & 0.340 & 0.022 & 0.006 & A \\
\hline 02.86 & 12590.36 & 12537.97 & 12642.75 & 53 & 6.576 & 0.161 & 0.834 & 0.339 & 0.047 & 0.009 & A \\
\hline 2003.09 & 12676.19 & 12643.77 & 12708.62 & 19 & 6.575 & 0.161 & 0.830 & 0.330 & 0.048 & 0.009 & A \\
\hline 2003.76 & 12915.93 & 12894.95 & 12936.92 & 34 & 6.531 & 0.236 & 0.833 & 0.332 & 0.074 & 0.007 & A \\
\hline 2004.01 & 13008.71 & 12945.81 & 13071.62 & 35 & 6.509 & 0.256 & 0.839 & 0.343 & 0.069 & 0.012 & A \\
\hline 2004.94 & 13348.26 & 13255.89 & 13440.62 & 68 & 6.581 & 0.273 & 0.826 & 0.319 & 0.088 & 0.008 & A \\
\hline \multicolumn{12}{|c|}{ V711 Tau } \\
\hline 1990.79 & 8181.93 & 8168.96 & 8194.89 & 23 & 5.774 & 0.082 & 0.886 & 0.461 & 0.024 & 0.006 & A \\
\hline 1990.89 & 8217.85 & 8199.89 & 8235.82 & 16 & 5.768 & 0.085 & 0.884 & 0.462 & 0.030 & 0.004 & A \\
\hline 1991.02 & 8266.19 & 8250.74 & 8281.64 & 10 & 5.771 & 0.070 & 0.888 & 0.466 & 0.022 & 0.002 & A \\
\hline 1991.14 & 8308.12 & 8290.66 & 8325.59 & 17 & 5.779 & 0.081 & 0.895 & 0.463 & 0.028 & 0.005 & A \\
\hline 1991.77 & 8540.39 & 8531.91 & 8548.86 & 13 & 5.818 & 0.059 & 0.894 & 0.461 & 0.020 & 0.007 & A \\
\hline 1991.85 & 8567.35 & 8551.91 & 8582.79 & 14 & 5.811 & 0.082 & 0.895 & 0.433 & 0.023 & 0.008 & A \\
\hline 1991.98 & 8614.74 & 8589.83 & 8639.65 & 9 & 5.815 & 0.094 & 0.891 & - & 0.035 & 0.011 & A \\
\hline 1992.10 & 8661.14 & 8640.68 & 8681.60 & 15 & 5.814 & 0.057 & 0.893 & 0.470 & 0.018 & 0.005 & $A+B$ \\
\hline 1993.79 & 9278.41 & 9261.89 & 9294.93 & 24 & 5.822 & 0.096 & 0.900 & 0.491 & 0.034 & 0.004 & $A+B$ \\
\hline 1993.90 & 9315.62 & 9297.80 & 9333.44 & 24 & 5.776 & 0.171 & 0.900 & 0.489 & 0.048 & 0.003 & $A+B$ \\
\hline 1994.09 & 9386.64 & 9355.69 & 9417.59 & 30 & 5.834 & 0.112 & 0.894 & 0.458 & 0.036 & 0.007 & $A+B$ \\
\hline 1995.76 & 9996.91 & 9981.89 & 10011.94 & 27 & 5.786 & 0.140 & 0.897 & 0.458 & 0.043 & 0.008 & A \\
\hline 1996.07 & 10110.66 & 10089.71 & 10131.61 & 17 & 5.820 & 0.068 & 0.899 & 0.449 & 0.022 & 0.008 & A \\
\hline 1996.91 & 10415.80 & 10391.91 & 10439.69 & 51 & 5.742 & 0.166 & 0.901 & 0.466 & 0.052 & 0.009 & $A+B$ \\
\hline
\end{tabular}


Table 4. continued.

\begin{tabular}{|c|c|c|c|c|c|c|c|c|c|c|c|}
\hline Mean epoch & $\mathrm{HJD}_{\text {mean }}$ & $\overline{\mathrm{HJD}_{\text {ini }}}$ & HJD $_{\text {end }}$ & $N_{\mathrm{m}}$ & $V_{\min }$ & $\Delta V$ & $B-V$ & $U-B$ & $\sigma_{v}$ & $\sigma_{c k 1-c}$ & Note \\
\hline 1997.06 & 10471.17 & 10440.71 & 10501.64 & 39 & 5.753 & 0.153 & 0.903 & 0.468 & 0.052 & 0.009 & $A+B$ \\
\hline 1997.81 & 10743.92 & 10726.87 & 10760.96 & 33 & 5.800 & 0.072 & 0.905 & 0.471 & 0.021 & 0.008 & A \\
\hline 1997.90 & 10779.33 & 10762.78 & 10795.88 & 28 & 5.808 & 0.075 & 0.906 & 0.473 & 0.021 & 0.008 & A \\
\hline 1997.99 & 10811.23 & 10796.74 & 10825.71 & 20 & 5.820 & 0.059 & 0.911 & 0.470 & 0.016 & 0.008 & A \\
\hline 1998.19 & 10882.62 & 10872.63 & 10892.61 & 11 & 5.796 & 0.049 & 0.900 & 0.469 & 0.018 & 0.008 & A \\
\hline 1998.74 & 11085.59 & 11061.56 & 11109.62 & 35 & 5.690 & 0.095 & 0.909 & 0.470 & 0.024 & 0.008 & $A+B$ \\
\hline 1998.89 & 11139.18 & 11114.89 & 11163.46 & 31 & 5.683 & 0.098 & 0.905 & 0.488 & 0.030 & 0.008 & $A+B$ \\
\hline 1999.08 & 11208.70 & 11181.74 & 11235.65 & 28 & 5.698 & 0.112 & 0.904 & 0.478 & 0.031 & 0.008 & A \\
\hline 1999.90 & 11508.32 & 11459.89 & 11556.74 & 48 & 5.725 & 0.111 & 0.906 & 0.474 & 0.037 & 0.012 & A \\
\hline 2000.14 & 11597.18 & 11572.74 & 11621.62 & 12 & 5.712 & 0.113 & 0.905 & 0.475 & 0.033 & 0.012 & A \\
\hline 2000.82 & 11844.34 & 11805.91 & 11882.77 & 39 & 5.671 & 0.150 & 0.901 & 0.478 & 0.047 & 0.007 & $A+B$ \\
\hline 2001.01 & 11913.56 & 11884.78 & 11942.34 & 31 & 5.667 & 0.154 & 0.904 & 0.483 & 0.050 & 0.007 & $A+B$ \\
\hline 2001.81 & 12205.23 & 12162.61 & 12247.84 & 31 & 5.736 & 0.117 & 0.912 & 0.487 & 0.037 & 0.007 & $A+B$ \\
\hline 2001.98 & 12267.76 & 12251.82 & 12283.70 & 6 & 5.757 & 0.075 & 0.909 & 0.491 & 0.028 & 0.008 & A \\
\hline 2002.81 & 12572.37 & 12537.94 & 12606.81 & 22 & 5.752 & 0.093 & 0.906 & 0.493 & 0.027 & 0.007 & A \\
\hline 2003.94 & 12985.28 & 12908.96 & 13061.61 & 14 & 5.789 & 0.125 & 0.912 & 0.505 & 0.039 & 0.009 & A \\
\hline 2004.84 & 13313.36 & 13259.00 & 13367.72 & 29 & 5.870 & 0.079 & 0.923 & 0.509 & 0.023 & 0.006 & A \\
\hline \multicolumn{12}{|c|}{ EI Eri } \\
\hline 1987.93 & 7136.73 & 7129.73 & 7143.72 & 13 & 7.011 & 0.040 & 0.670 & 0.164 & 0.013 & - & $\mathrm{C}$ \\
\hline 1989.96 & 7879.24 & 7869.74 & 7888.74 & 10 & 6.996 & 0.073 & 0.673 & 0.145 & 0.030 & - & $\mathrm{C}$ \\
\hline 1990.84 & 8199.42 & 8180.96 & 8217.88 & 20 & 6.970 & 0.081 & 0.655 & 0.093 & 0.029 & 0.009 & A \\
\hline 1991.11 & 8297.17 & 8268.75 & 8325.60 & 17 & 7.020 & 0.044 & 0.669 & 0.173 & 0.015 & 0.007 & A \\
\hline 1991.82 & 8555.40 & 8536.95 & 8573.85 & 18 & 6.995 & 0.083 & 0.669 & - & 0.025 & 0.006 & A \\
\hline 1992.08 & 8652.17 & 8623.71 & 8680.63 & 15 & 7.012 & 0.048 & 0.671 & 0.099 & 0.011 & 0.008 & A \\
\hline 1992.80 & 8914.40 & 8897.92 & 8930.87 & 13 & 6.958 & 0.130 & 0.658 & 0.221 & 0.041 & 0.006 & $A+B$ \\
\hline 1993,03 & 8944.80 & 9056.60 & 9000.00 & 24 & 6.968 & 0.065 & 0.666 & 0.123 & 0.041 & 0.007 & $A+B$ \\
\hline 1993.76 & 9264.97 & 9247.95 & 9281.99 & 16 & 7.016 & 0.106 & 0.674 & 0.192 & 0.041 & 0.008 & $A+B$ \\
\hline 1993.90 & 9317.78 & 9284.87 & 9350.69 & 63 & 6.998 & 0.094 & 0.678 & 0.125 & 0.029 & 0.008 & $A+B$ \\
\hline 1994.05 & 9373.00 & 9327.39 & 9418.61 & 25 & 6.966 & 0.151 & 0.675 & 0.189 & 0.049 & 0.008 & $A+B$ \\
\hline 1994.94 & 9697.44 & 9643.53 & 9751.35 & 11 & 7.035 & 0.075 & 0.691 & 0.141 & 0.025 & 0.008 & $A+B$ \\
\hline 1995.86 & 10030.87 & 9982.99 & 10078.76 & 42 & 7.060 & 0.057 & 0.673 & 0.145 & 0.013 & 0.006 & A \\
\hline 1996.06 & 10105.71 & 10087.73 & 10123.68 & 22 & 7.048 & 0.078 & 0.674 & 0.098 & 0.025 & 0.006 & A \\
\hline 1996.90 & 10412.92 & 10392.95 & 10432.89 & 27 & 7.000 & 0.087 & 0.670 & 0.099 & 0.026 & 0.006 & A \\
\hline 1997.01 & 10452.80 & 10434.88 & 10470.72 & 16 & 6.995 & 0.070 & 0.664 & 0.101 & 0.021 & 0.006 & A \\
\hline 1997.12 & 10495.69 & 10477.73 & 10513.65 & 24 & 6.978 & 0.074 & 0.668 & 0.090 & 0.019 & 0.006 & A \\
\hline 1997.77 & 10730.91 & 10718.86 & 10742.96 & 25 & 7.006 & 0.042 & 0.663 & 0.100 & 0.012 & 0.008 & A \\
\hline 1997.87 & 10766.85 & 10749.91 & 10783.79 & 40 & 6.973 & 0.093 & 0.664 & 0.088 & 0.023 & 0.008 & $A+B$ \\
\hline 1998.05 & 10832.55 & 10808.42 & 10856.67 & 26 & 6.997 & 0.078 & 0.670 & 0.120 & 0.022 & 0.008 & $A+B$ \\
\hline 1998.76 & 11092.92 & 11078.97 & 11106.86 & 16 & 7.030 & 0.041 & 0.663 & 0.110 & 0.014 & 0.007 & $A+B$ \\
\hline 1998.87 & 11133.91 & 11115.90 & 11151.92 & 21 & 7.016 & 0.049 & 0.661 & 0.103 & 0.016 & 0.007 & $A+B$ \\
\hline 1999.04 & 11193.74 & 11181.77 & 11205.71 & 8 & 6.987 & 0.093 & 0.663 & 0.104 & 0.037 & 0.007 & $A+B$ \\
\hline 1999.11 & 11222.20 & 11206.72 & 11237.68 & 11 & 7.010 & 0.059 & 0.668 & 0.104 & 0.021 & 0.007 & A \\
\hline 1999.82 & 11477.72 & 11448.91 & 11506.53 & 24 & 6.961 & 0.070 & 0.666 & 0.099 & 0.019 & 0.009 & $A+B$ \\
\hline 2000.03 & 11556.58 & 11531.42 & 11581.73 & 21 & 7.011 & 0.064 & 0.680 & 0.127 & 0.021 & .009 & $A+B$ \\
\hline 2000.16 & 11604.17 & 11584.72 & 11623.62 & 12 & 6.971 & 0.088 & 0.666 & 0.085 & 0.026 & 0.009 & A \\
\hline 2000.91 & 11878.77 & 11832.87 & 11924.68 & 24 & 6.967 & 0.070 & 0.659 & 0.097 & 0.017 & 0.007 & A \\
\hline 2001.10 & 11949.49 & 11928.34 & 11970.64 & 17 & 6.972 & 0.069 & 0.671 & 0.085 & 0.017 & 0.007 & $A+B$ \\
\hline 2001.81 & 12207.02 & 12166.64 & 12247.40 & 41 & 6.961 & 0.089 & 0.666 & 0.131 & 0.026 & 0.007 & $A+B$ \\
\hline 2001.99 & 12269.77 & 12249.83 & 12289.71 & 14 & 6.953 & 0.102 & 0.654 & 0.084 & 0.036 & 0.007 & A \\
\hline 2002.13 & 12322.66 & 12299.71 & 12345.61 & 13 & 6.988 & 0.046 & 0.659 & 0.086 & 0.015 & 0.006 & A \\
\hline 2002.77 & 12555.86 & 12538.90 & 12572.83 & 14 & 6.921 & 0.110 & 0.658 & 0.087 & 0.031 & 0.006 & A \\
\hline 2002.94 & 12619.25 & 12595.78 & 12642.72 & 22 & 6.925 & 0.085 & 0.657 & 0.077 & 0.026 & 0.008 & A \\
\hline 2003.07 & 12668.13 & 12643.65 & 12692.60 & 22 & 6.942 & 0.077 & 0.653 & 0.074 & 0.020 & 0.007 & A \\
\hline 2003.75 & 12913.92 & 12894.93 & 12932.92 & 28 & 6.976 & 0.049 & 0.652 & 0.069 & 0.016 & 0.007 & A \\
\hline 2003.86 & 12953.39 & 12933.93 & 12972.85 & 33 & 6.968 & 0.067 & 0.648 & 0.073 & 0.019 & 0.015 & A \\
\hline 2003.96 & 12990.75 & 12973.73 & 13007.77 & 22 & 6.977 & 0.048 & 0.649 & 0.071 & 0.015 & 0.012 & A \\
\hline 2004.06 & 13028.15 & 13011.69 & 13044.62 & 18 & 6.949 & 0.123 & 0.640 & 0.067 & 0.036 & 0.021 & A \\
\hline 2004.17 & 13067.61 & 13055.62 & 13079.60 & 9 & 6.977 & 0.110 & 0.634 & 0.060 & 0.033 & 0.015 & A \\
\hline 2004.76 & 13284.37 & 13259.96 & 13308.78 & 33 & 6.953 & 0.159 & 0.652 & 0.069 & 0.051 & 0.010 & A \\
\hline 2004.88 & 13328.81 & 13309.80 & 13347.82 & 30 & 6.929 & 0.157 & 0.653 & 0.082 & 0.051 & 0.010 & A \\
\hline 2005.07 & 13398.65 & 13350.70 & 13446.60 & 42 & 6.949 & 0.104 & 0.653 & 0.085 & 0.029 & 0.010 & A \\
\hline \multicolumn{12}{|c|}{ V1149 Ori } \\
\hline 1989.12 & 7572.15 & 7548.72 & 7595.57 & 12 & 6.788 & 0.130 & 1.163 & 0.947 & 0.043 & 0.007 & $\mathrm{C}$ \\
\hline 1990.92 & 8219.54 & 8183.00 & 8276.75 & 19 & 6.781 & 0.134 & 1.137 & 0.956 & 0.050 & 0.007 & A \\
\hline 1991.15 & 8311.05 & 8291.73 & 8332.64 & 14 & 6.799 & 0.114 & 1.143 & 0.959 & 0.036 & 0.008 & A \\
\hline 1991.86 & 8563.60 & 8537.00 & 8606.92 & 22 & 6.767 & 0.188 & 1.139 & 0.975 & 0.062 & 0.007 & A \\
\hline
\end{tabular}


Table 4. continued.

\begin{tabular}{|c|c|c|c|c|c|c|c|c|c|c|c|}
\hline Mean epoch & $\mathrm{HJD}_{\text {mean }}$ & $\mathrm{HJD}_{\text {ini }}$ & $\mathrm{HJD}_{\text {end }}$ & $N_{\mathrm{m}}$ & $V_{\min }$ & $\overline{\Delta V}$ & $B-V$ & $\overline{U-B}$ & $\sigma_{v}$ & $\sigma_{c k 1-c}$ & Note \\
\hline 1992.09 & 8657.49 & 8630.84 & 8678.67 & 9 & 6.811 & 0.048 & 1.135 & 0.939 & 0.019 & 0.007 & $\mathrm{~A}$ \\
\hline 1992.80 & 8916.86 & 8896.93 & 8933.99 & 20 & 6.718 & 0.247 & 1.141 & 0.965 & 0.079 & 0.010 & A \\
\hline 1993.01 & 8994.02 & 8957.82 & 9024.42 & 31 & 6.754 & 0.158 & 1.155 & 0.949 & 0.042 & 0.010 & $A+B+C$ \\
\hline 1993.83 & 9290.99 & 9247.99 & 9337.85 & 26 & 6.811 & 0.149 & 1.152 & 0.986 & 0.042 & 0.007 & $\mathrm{~A}+\mathrm{B}$ \\
\hline 1994.06 & 9370.60 & 9353.43 & 9398.75 & 20 & 6.802 & 0.130 & 1.149 & 0.989 & 0.038 & 0.007 & $A+B$ \\
\hline 1994.18 & 9423.65 & 9412.68 & 9417.96 & 7 & 6.908 & 0.039 & 1.146 & 0.995 & 0.014 & 0.010 & A \\
\hline 1994.99 & 9714.49 & 9643.63 & 9785.34 & 15 & 6.828 & 0.134 & 1.147 & 0.941 & 0.041 & 0.009 & B \\
\hline 1995.82 & 10019.42 & 10000.89 & 10037.95 & 40 & 6.800 & 0.113 & 1.159 & 0.956 & 0.032 & 0.007 & $A+B$ \\
\hline 1995.96 & 10068.71 & 10043.66 & 10093.76 & 40 & 6.793 & 0.156 & 1.160 & 0.950 & 0.052 & 0.007 & $A+B$ \\
\hline 1996.14 & 10133.69 & 10096.76 & 10170.62 & 33 & 6.790 & 0.177 & 1.150 & 0.935 & 0.057 & 0.007 & $A+B$ \\
\hline 1996.92 & 10421.43 & 10391.99 & 10450.88 & 68 & 6.689 & 0.179 & 1.152 & 0.949 & 0.061 & 0.008 & $A+B$ \\
\hline 1997.14 & 10500.56 & 10464.84 & 10536.29 & 38 & 6.690 & 0.208 & 1.152 & 0.952 & 0.075 & 0.008 & $A+B$ \\
\hline 1997.86 & 10762.93 & 10718.92 & 10806.94 & 49 & 6.810 & 0.277 & 1.165 & 0.963 & 0.088 & 0.007 & A \\
\hline 1998.14 & 10868.09 & 10814.56 & 10921.62 & 44 & 6.854 & 0.246 & 1.170 & 0.956 & 0.087 & 0.007 & $A+B$ \\
\hline 1998.79 & 11103.42 & 11081.96 & 11124.88 & 21 & 6.835 & 0.196 & 1.173 & 0.954 & 0.061 & 0.008 & A \\
\hline 1998.98 & 11172.41 & 11132.04 & 11212.78 & 30 & 6.818 & 0.209 & 1.172 & 0.965 & 0.066 & 0.008 & A \\
\hline 1999.19 & 11247.22 & 11218.80 & 11275.65 & 22 & 6.838 & 0.168 & 1.169 & 0.972 & 0.056 & 0.008 & A \\
\hline 1999.86 & 11493.43 & 11447.97 & 11538.89 & 46 & 6.826 & 0.180 & 1.176 & 0.963 & 0.060 & 0.009 & $A+B$ \\
\hline 2000.12 & 11590.76 & 11545.88 & 11635.64 & 29 & 6.854 & 0.172 & 1.171 & 0.968 & 0.053 & 0.009 & $A+B$ \\
\hline 2000.78 & 11830.81 & 11803.63 & 11858.00 & 23 & 6.861 & 0.141 & 1.161 & 0.974 & 0.052 & 0.005 & $A+B$ \\
\hline 2001.03 & 11920.58 & 11876.83 & 11964.33 & 58 & 6.825 & 0.186 & 1.162 & 0.975 & 0.061 & 0.005 & $A+B$ \\
\hline 2001.18 & 11975.68 & 11953.73 & 11997.63 & 19 & 6.816 & 0.118 & 1.155 & 0.986 & 0.040 & 0.005 & A \\
\hline 2001.84 & 12219.28 & 12193.01 & 12245.55 & 21 & 6.818 & 0.083 & 1.160 & 0.975 & 0.026 & 0.008 & $A+B$ \\
\hline 2002.02 & 12284.29 & 12261.88 & 12306.71 & 25 & 6.790 & 0.109 & 1.155 & 0.972 & 0.035 & 0.008 & A \\
\hline 2002.17 & 12335.17 & 12311.70 & 12358.64 & 28 & 6.791 & 0.116 & 1.160 & 0.934 & 0.042 & 0.008 & A \\
\hline 2002.80 & 12566.92 & 12538.00 & 12595.85 & 45 & 6.739 & 0.151 & 1.152 & 0.973 & 0.041 & 0.008 & A \\
\hline 2002.98 & 12633.22 & 12601.76 & 12664.68 & 52 & 6.680 & 0.213 & 1.155 & 0.973 & 0.054 & 0.010 & A \\
\hline 2003.17 & 12700.65 & 12665.68 & 12735.62 & 36 & 6.673 & 0.198 & 1.141 & 0.969 & 0.054 & 0.011 & A \\
\hline 2003.86 & 12953.37 & 12895.00 & 13011.74 & ** & 6.629 & 0.311 & 1.150 & 0.979 & 0.103 & 0.009 & A \\
\hline 2004.13 & 13054.19 & 13013.74 & 13094.63 & 45 & 6.663 & 0.248 & 1.143 & 0.977 & 0.086 & 0.022 & A \\
\hline 2004.97 & 13358.78 & 13255.93 & 13461.62 & $* *$ & 6.593 & 0.244 & 1.139 & 0.999 & 0.072 & 0.010 & A \\
\hline \multicolumn{12}{|c|}{ DH Leo } \\
\hline 1993.30 & 9095.82 & 9067.30 & 9124.34 & 21 & 7.848 & 0.033 & 0.898 & 0.485 & 0.010 & 0.008 & B \\
\hline 1995.17 & 9777.96 & 9743.55 & 9812.37 & 18 & 7.811 & 0.079 & 0.899 & 0.478 & 0.027 & 0.01 & B \\
\hline 1997.14 & 10501.58 & 10481.58 & 10521.58 & 9 & 7.830 & 0.037 & 0.883 & 0.473 & 0.010 & 0.008 & B \\
\hline 1997.30 & 10560.36 & 10548.33 & 10572.40 & 9 & 7.850 & 0.049 & 0.875 & 0.484 & 0.014 & 0.008 & B \\
\hline 1997.37 & 10585.33 & 10577.34 & 10593.32 & 12 & 7.804 & 0.138 & 0.885 & 0.477 & 0.049 & 0.010 & B \\
\hline 1998.04 & 10829.15 & 10814.63 & 10843.67 & 10 & 7.792 & 0.125 & 0.889 & 0.498 & 0.043 & 0.011 & B \\
\hline 1998.35 & 10939.85 & 10930.39 & 10949.31 & 8 & 7.813 & 0.063 & 0.897 & 0.507 & 0.023 & 0.008 & B \\
\hline 1998.86 & 11128.64 & 11121.58 & 11135.70 & 6 & 7.825 & 0.092 & 0.917 & 0.495 & 0.034 & 0.011 & B \\
\hline 1999.07 & 11206.51 & 11186.52 & 11226.50 & 10 & 7.836 & 0.064 & 0.920 & 0.428 & 0.018 & 0.010 & B \\
\hline 2000.09 & 11580.03 & 11574.56 & 11585.49 & 5 & 7.922 & 0.022 & 0.892 & - & 0.009 & 0.008 & B \\
\hline 2000.24 & 11632.86 & 11614.41 & 11651.32 & 8 & 7.795 & 0.176 & 0.912 & 0.452 & 0.073 & 0.011 & B \\
\hline 2000.31 & 11657.87 & 11654.29 & 11661.45 & 26 & 7.805 & 0.142 & 0.903 & 0.474 & 0.042 & 0.008 & B \\
\hline 2001.11 & 11949.62 & 11934.66 & 11964.57 & 42 & 7.855 & 0.066 & 0.910 & 0.503 & 0.019 & 0.009 & B \\
\hline \multicolumn{12}{|c|}{ HU Vir } \\
\hline 1989.12 & 7571.81 & 7548.86 & 7594.76 & 23 & 8.669 & 0.147 & 0.999 & 0.639 & 0.051 & 0.007 & $\mathrm{C}$ \\
\hline 1990.18 & 7955.70 & 7949.73 & 7961.67 & 13 & 8.549 & 0.228 & 1.023 & 0.631 & 0.081 & 0.008 & $\mathrm{C}$ \\
\hline 1991.11 & 8299.88 & 8268.95 & 8330.80 & 22 & 8.577 & 0.244 & 1.003 & 0.631 & 0.074 & 0.010 & B \\
\hline 1991.23 & 8339.70 & 8331.76 & 8347.64 & 20 & 8.587 & 0.248 & 1.028 & 0.605 & 0.074 & 0.010 & $\mathrm{C}$ \\
\hline 1991.34 & 8379.19 & 8350.73 & 8407.66 & 29 & 8.603 & 0.237 & 1.003 & 0.642 & 0.071 & 0.008 & B \\
\hline 1992.04 & 8638.48 & 8599.02 & 8677.93 & 57 & 8.663 & 0.219 & 0.937 & 0.631 & 0.061 & 0.011 & $A+B$ \\
\hline 1992.22 & 8702.75 & 8678.78 & 8726.71 & 47 & 8.708 & 0.194 & 0.991 & 0.648 & 0.056 & 0.010 & $A+B$ \\
\hline 1992.39 & 8765.65 & 8732.66 & 8798.65 & 60 & 8.733 & 0.265 & 1.014 & 0.675 & 0.071 & 0.012 & $A+B$ \\
\hline 1993.03 & 8999.49 & 8954.04 & 9044.93 & 76 & 8.701 & 0.166 & 0.946 & 0.622 & 0.043 & 0.009 & $A+B$ \\
\hline 1993.25 & 9079.79 & 9051.82 & 9107.76 & 97 & 8.681 & 0.194 & 0.998 & 0.618 & 0.050 & 0.010 & $A+B$ \\
\hline 1993.41 & 9137.15 & 9113.65 & 9160.65 & 56 & 8.682 & 0.215 & 1.003 & 0.626 & 0.062 & 0.009 & $A+B$ \\
\hline 1994.02 & 9360.47 & 9335.01 & 9385.94 & 68 & 8.681 & 0.149 & 1.005 & 0.636 & 0.036 & 0.009 & $A+B$ \\
\hline 1994.22 & 9431.81 & 9386.86 & 9476.75 & $* *$ & 8.711 & 0.118 & 1.001 & 0.632 & 0.029 & 0.010 & $A+B$ \\
\hline 1994.40 & 9497.66 & 9477.68 & 9517.65 & 29 & 8.683 & 0.144 & 0.999 & 0.650 & 0.032 & 0.011 & $A+B$ \\
\hline 1995.05 & 9739.29 & 9732.80 & 9745.79 & 12 & 8.642 & 0.136 & 0.996 & 0.608 & 0.056 & 0.009 & $\mathrm{C}$ \\
\hline 1995.46 & 9885.65 & 9877.65 & 9893.65 & 20 & 8.642 & 0.197 & 1.006 & 0.608 & 0.071 & 0.012 & $A+B$ \\
\hline 1996.11 & 10124.41 & 10078.02 & 10170.81 & 63 & 8.589 & 0.274 & 0.997 & 0.622 & 0.074 & 0.009 & $A+B$ \\
\hline 1996.32 & 10202.18 & 10187.67 & 10216.69 & 24 & 8.666 & 0.172 & 1.000 & 0.638 & 0.054 & 0.009 & A \\
\hline 1996.42 & 10236.21 & 10218.76 & 10253.66 & 25 & 8.663 & 0.155 & 1.006 & 0.631 & 0.051 & 0.009 & A \\
\hline 1996.93 & 10423.47 & 10409.00 & 10437.94 & 18 & 8.629 & 0.184 & 1.004 & 0.620 & 0.061 & 0.012 & $A+B$ \\
\hline
\end{tabular}


Table 4. continued.

\begin{tabular}{|c|c|c|c|c|c|c|c|c|c|c|c|}
\hline Mean epoch & $\mathrm{HJD}_{\text {mean }}$ & $\mathrm{HJD}_{\text {ini }}$ & $\mathrm{HJD}_{\text {end }}$ & $N_{\mathrm{m}}$ & $V_{\min }$ & $\overline{\Delta V}$ & $B-V$ & $U-B$ & $\sigma_{v}$ & $\sigma_{c k 1-c}$ & Note \\
\hline 1997.09 & 10483.71 & 10467.54 & 10499.88 & 30 & 8.677 & 0.193 & 1.001 & 0.638 & 0.045 & 0.012 & $\mathrm{~A}+\mathrm{B}$ \\
\hline 1997.21 & 10525.33 & 10500.91 & 10549.75 & 45 & 8.699 & 0.123 & 1.012 & 0.654 & 0.040 & 0.012 & $A+B$ \\
\hline 1997.32 & 10566.70 & 10550.67 & 10582.72 & 32 & 8.681 & 0.141 & 1.014 & 0.664 & 0.045 & 0.012 & A \\
\hline 1997.43 & 10606.66 & 10590.66 & 10622.66 & 24 & 8.632 & 0.282 & 1.019 & 0.663 & 0.094 & 0.012 & A \\
\hline 1997.93 & 10789.03 & 10768.02 & 10810.05 & 29 & 8.623 & 0.407 & 1.020 & 0.661 & 0.128 & 0.009 & A \\
\hline 1998.11 & 10856.31 & 10814.91 & 10897.72 & 66 & 8.620 & 0.419 & 1.027 & 0.654 & 0.119 & 0.009 & A \\
\hline 1998.29 & 10919.22 & 10903.76 & 10934.68 & 41 & 8.698 & 0.360 & 1.028 & 0.672 & 0.113 & 0.009 & A \\
\hline 1998.42 & 10965.67 & 10936.67 & 10994.67 & 34 & 8.693 & 0.338 & 1.021 & 0.648 & 0.132 & 0.009 & $A+B$ \\
\hline 1998.93 & 11155.46 & 11133.02 & 11177.90 & 22 & 8.717 & 0.227 & 1.016 & 0.639 & 0.075 & 0.013 & A \\
\hline 1999.05 & 11199.37 & 11181.90 & 11216.83 & 35 & 8.741 & 0.221 & 1.015 & 0.649 & 0.068 & 0.013 & A \\
\hline 1999.21 & 11254.84 & 11222.87 & 11286.81 & 44 & 8.756 & 0.188 & 1.017 & 0.643 & 0.057 & 0.013 & A \\
\hline 1999.40 & 11324.17 & 11287.68 & 11360.67 & 53 & 8.787 & 0.166 & 1.024 & 0.658 & 0.052 & 0.013 & A \\
\hline 1999.93 & 11519.03 & 11504.01 & 11534.04 & 18 & 8.789 & 0.299 & 1.020 & 0.662 & 0.088 & 0.012 & A \\
\hline 2000.03 & 11557.43 & 11536.03 & 11578.84 & 33 & 8.824 & 0.237 & 1.022 & 0.671 & 0.061 & 0.012 & $A+B$ \\
\hline 2000.13 & 11593.86 & 11579.85 & 11607.88 & 18 & 8.808 & 0.277 & 1.029 & 0.679 & 0.108 & 0.012 & A \\
\hline 2000.24 & 11633.24 & 11612.76 & 11653.71 & 42 & 8.769 & 0.288 & 1.018 & 0.644 & 0.084 & 0.012 & $A+B$ \\
\hline 2000.40 & 11689.19 & 11666.70 & 11711.67 & 26 & 8.726 & 0.342 & 1.026 & 0.655 & 0.127 & 0.012 & A \\
\hline 2000.97 & 11899.01 & 11862.02 & 11935.99 & 47 & 8.655 & 0.407 & 1.021 & 0.662 & 0.145 & 0.011 & $A+B$ \\
\hline 2001.17 & 11970.32 & 11943.83 & 11996.82 & 51 & 8.645 & 0.385 & 1.010 & 0.658 & 0.116 & 0.011 & $A+B$ \\
\hline 2001.30 & 12020.27 & 12001.78 & 12038.76 & 34 & 8.655 & 0.343 & 1.006 & 0.643 & 0.108 & 0.011 & $A+B$ \\
\hline 2001.42 & 12060.67 & 12044.67 & 12076.67 & 29 & 8.663 & 0.288 & 1.012 & 0.652 & 0.085 & 0.011 & $A+B$ \\
\hline 2002.04 & 12289.91 & 12251.02 & 12328.80 & 79 & 8.684 & 0.402 & 1.014 & 0.670 & 0.138 & 0.015 & A \\
\hline 2002.23 & 12359.25 & 12331.75 & 12386.75 & 70 & 8.642 & 0.414 & 1.022 & 0.659 & 0.129 & 0.015 & $A+B$ \\
\hline 2002.38 & 12413.69 & 12390.73 & 12436.66 & 58 & 8.660 & 0.369 & 1.026 & 0.660 & 0.112 & 0.015 & $A+B$ \\
\hline 2002.94 & 12617.46 & 12592.03 & 12642.89 & 31 & 8.673 & 0.279 & 1.012 & 0.677 & 0.092 & 0.013 & A \\
\hline 2003.10 & 12678.85 & 12643.94 & 12713.76 & 58 & 8.660 & 0.257 & 1.010 & 0.644 & 0.081 & 0.013 & A \\
\hline 2003.27 & 12737.25 & 1271 & 12755 & 38 & 8.612 & 0.291 & 1.011 & 0.657 & 0.084 & 0.012 & A \\
\hline 2003.42 & 12791.66 & 127 & 128 & 38 & 8.616 & 0.313 & 1.026 & 0.659 & 87 & 010 & A \\
\hline 2003 & 12992.44 & 129 & 130 & 50 & 8.659 & 0.255 & 1.021 & 0.671 & 65 & 0.016 & A \\
\hline 200 & 13062.29 & 130 & $130 s$ & 47 & 8.689 & 0.226 & 1.021 & 0.658 & 0.052 & 0.011 & A \\
\hline 2004 & 13117.17 & 1309 & 1313 & 37 & 8.706 & 0.147 & 1.028 & 0.645 & 0.047 & 0.023 & A \\
\hline 2005 & 13390.92 & 13323.03 & 1345 & 49 & 8.746 & 0.140 & 1.026 & 0.673 & 0.038 & 0.018 & A \\
\hline \multicolumn{12}{|c|}{ CVn } \\
\hline 1990.23 & 7976.34 & 7897.01 & 8055.67 & 63 & 8.057 & 0.142 & 0.589 & 0.079 & 0.034 & 0.011 & A \\
\hline 1991.24 & 8343.34 & 8245.02 & 8441.65 & 104 & 8.060 & 1.103 & 0.609 & 0.084 & 0.256 & 0.010 & A \\
\hline 1992.19 & 8692.41 & 8605.04 & 8779.79 & 66 & 8.036 & 0.758 & 0.596 & 0.082 & 0.129 & 0.010 & A \\
\hline 1993.24 & 9073.22 & 8964.05 & 9182.38 & 120 & 8.008 & 1.160 & 0.612 & 0.093 & 0.195 & 0.010 & $A+B$ \\
\hline 1994.24 & 9438.84 & 9335.04 & 9542.65 & 121 & 8.013 & 1.104 & 0.619 & 0.092 & 0.249 & 0.011 & $A+B$ \\
\hline 1995.33 & 9837.53 & 9770.40 & 9904.66 & 93 & 7.973 & 1.003 & 0.661 & 0.156 & 0.328 & 0.007 & $A+B$ \\
\hline 96.19 & 10153.84 & 10091.99 & 10215.69 & 24 & 7.971 & 0.977 & 0.627 & 0.123 & 0.260 & 0.009 & $A+B$ \\
\hline 97.03 & 10462.40 & 10405.04 & 10519.76 & 92 & 7.975 & 1.145 & 0.624 & 0.099 & 0.259 & 0.008 & $A+B$ \\
\hline & 10578.73 & 10520.78 & 10636.67 & 122 & 7.977 & 1.192 & 0.627 & 0.109 & 0.285 & 0.008 & $A+B$ \\
\hline & 884.86 & 10772 & 10997 & 209 & 7.948 & 1.310 & 0.618 & 0.102 & 0.251 & 0.008 & $A+B$ \\
\hline & 75 & 111 & 1122 & 80 & 946 & 62 & 0.614 & 0.083 & 32 & 09 & $A+B$ \\
\hline & 61 & 112 & 1137 & 119 & 7.927 & 1.417 & 0.621 & 0.095 & 0.290 & 0.009 & $A+B$ \\
\hline 2000.04 & 8.96 & 11505.03 & 11612.89 & 76 & 7.964 & 1.258 & 0.636 & 0.107 & 0.337 & 0.009 & $A+B$ \\
\hline 2000.35 & 11670.52 & 11614.63 & 11726.40 & 93 & 7.947 & 1.163 & 0.622 & 0.113 & 0.254 & 0.009 & $A+B$ \\
\hline 2001.42 & 12061.54 & 11872.03 & 12251.05 & 128 & 7.941 & 0.981 & 0.637 & 0.122 & 0.254 & 0.008 & $A+B$ \\
\hline 2002.29 & 12381.84 & 12314.94 & 12448.74 & 108 & 7.934 & 0.880 & 0.614 & 0.108 & 0.169 & 0.008 & A \\
\hline 2003.22 & 12718.33 & 12613.00 & 12823.66 & 137 & 7.962 & 1.032 & 0.628 & 0.111 & 0.259 & 0.016 & A \\
\hline 2004.08 & 13033.96 & 12972.02 & 13095.90 & 47 & 7.976 & 1.074 & 0.633 & 0.123 & 0.287 & 0.014 & A \\
\hline 2004.76 & 13282.22 & 13102.75 & 13461.69 & 98 & 7.981 & 1.271 & 0.613 & 0.091 & 0.248 & 0.015 & A \\
\hline \multicolumn{12}{|c|}{ V775 H } \\
\hline 1990.40 & 8039.40 & 8032.86 & 8045.94 & 6 & 8.091 & 0.163 & 0.911 & - & 0.065 & 0.012 & A \\
\hline 1990.82 & 8192.62 & 8173.66 & 8211.57 & 18 & 8.159 & 0.086 & 0.919 & 0.627 & 0.028 & 0.012 & A \\
\hline 1991.30 & 8365.97 & 8359.97 & 8371.97 & 10 & 8.135 & 0.081 & 0.906 & 0.593 & 0.028 & 0.009 & A \\
\hline 1991.42 & 8409.44 & 8387.90 & 8430.98 & 21 & 8.144 & 0.107 & 0.909 & 0.613 & 0.035 & 0.008 & A \\
\hline 1991.80 & 8549.13 & 8530.66 & 8567.59 & 17 & 8.179 & 0.098 & 0.908 & - & 0.033 & 0.008 & A \\
\hline 1992.35 & 8749.40 & 8718.97 & 8779.82 & 26 & 8.159 & 0.160 & 0.910 & 0.609 & 0.051 & 0.008 & A \\
\hline 1992.47 & 8795.92 & 8781.92 & 8809.93 & 20 & 8.156 & 0.147 & 0.907 & 0.594 & 0.043 & 0.010 & A \\
\hline 1992.84 & 8929.10 & 8911.63 & 8946.56 & 15 & 8.197 & 0.093 & 0.919 & 0.632 & 0.031 & 0.010 & A \\
\hline 1993.32 & 9106.45 & 9101.93 & 9110.97 & 9 & 8.245 & 0.057 & 0.921 & 0.655 & 0.020 & 0.010 & A \\
\hline 1993.48 & 9161.86 & 9127.89 & 9195.84 & 28 & 8.208 & 0.145 & 0.921 & 0.665 & 0.055 & 0.010 & A \\
\hline 1994.02 & 9360.54 & 9219.53 & 9501.54 & 19 & 8.204 & 0.110 & 0.923 & 0.630 & 0.036 & 0.012 & $\mathrm{~A}+\mathrm{B}$ \\
\hline & 534.57 & 9505.59 & 9563.54 & 23 & 8.226 & 0.066 & 0.918 & 0.634 & 0.017 & 0.010 & $A+B$ \\
\hline 1995.45 & 9881.72 & 9853.56 & 9909.88 & 31 & 8.211 & 0.090 & 0.921 & 0.674 & 0.027 & 0.008 & $A+B$ \\
\hline
\end{tabular}


Table 4. continued.

\begin{tabular}{|c|c|c|c|c|c|c|c|c|c|c|c|}
\hline Mean epoch & $\mathrm{HJD}_{\text {mean }}$ & $\mathrm{HJD}_{\text {ini }}$ & $\mathrm{HJD}_{\text {end }}$ & $N_{\mathrm{m}}$ & $V_{\min }$ & $\Delta V$ & $B-V$ & $U-B$ & $\sigma_{v}$ & $\sigma_{c k 1-c}$ & Note \\
\hline 1995.79 & 10006.60 & 9982.63 & 10030.56 & 25 & 8.225 & 0.055 & 0.917 & 0.643 & 0.015 & 0.007 & $A+B$ \\
\hline 1996.48 & 10257.70 & 10210.86 & 10304.54 & 20 & 8.128 & 0.116 & 0.914 & 0.628 & 0.029 & 0.010 & $A+B$ \\
\hline 1997.17 & 10512.50 & 10498.04 & 10526.96 & 18 & 8.147 & 0.092 & 0.914 & 0.621 & 0.029 & 0.008 & A \\
\hline 1997.29 & 10556.43 & 10527.95 & 10584.91 & 34 & 8.131 & 0.084 & 0.911 & 0.611 & 0.021 & 0.008 & A \\
\hline 1997.42 & 10599.87 & 10590.77 & 10608.97 & 18 & 8.117 & 0.107 & 0.915 & 0.610 & 0.035 & 0.008 & A \\
\hline 1997.47 & 10620.38 & 10609.83 & 10630.93 & 31 & 8.079 & 0.150 & 0.911 & 0.630 & 0.039 & 0.008 & A \\
\hline 1997.60 & 10667.01 & 10644.54 & 10689.47 & 17 & 8.121 & 0.096 & 0.904 & 0.610 & 0.033 & 0.008 & B \\
\hline 1997.76 & 10726.47 & 10704.29 & 10748.64 & 22 & 8.128 & 0.098 & 0.913 & 0.635 & 0.028 & 0.006 & $A+B$ \\
\hline 1997.87 & 10766.59 & 10751.62 & 10781.56 & 13 & 8.120 & 0.124 & 0.914 & 0.629 & 0.049 & 0.006 & A \\
\hline 1998.17 & 10877.50 & 10857.05 & 10897.95 & 18 & 8.135 & 0.054 & 0.906 & 0.616 & 0.015 & 0.007 & A \\
\hline 1998.28 & 10914.90 & 10903.94 & 10925.86 & 19 & 8.101 & 0.089 & 0.906 & 0.593 & 0.029 & 0.007 & A \\
\hline 1998.36 & 10943.91 & 10929.87 & 10957.94 & 14 & 8.101 & 0.110 & 0.897 & 0.627 & 0.039 & 0.007 & A \\
\hline 1998.61 & 11037.51 & 10966.79 & 11108.24 & 32 & 8.105 & 0.090 & 0.904 & 0.619 & 0.029 & 0.007 & $A+B$ \\
\hline 1998.86 & 11129.10 & 11115.64 & 11142.57 & 20 & 8.067 & 0.119 & 0.903 & 0.612 & 0.041 & 0.007 & A \\
\hline 1999.15 & 11237.04 & 11223.05 & 11251.03 & 10 & 8.066 & 0.127 & 0.892 & 0.595 & 0.047 & 0.007 & A \\
\hline 1999.27 & 11277.41 & 11257.97 & 11296.85 & 21 & 8.045 & 0.120 & 0.897 & 0.594 & 0.033 & 0.007 & $A+B$ \\
\hline 1999.38 & 11316.37 & 11300.85 & 11331.90 & 25 & 8.063 & 0.093 & 0.902 & 0.593 & 0.030 & 0.007 & $A+B$ \\
\hline 1999.47 & 11348.90 & 11334.83 & 11362.96 & 18 & 8.035 & 0.125 & 0.903 & 0.616 & 0.036 & 0.007 & A \\
\hline 1999.77 & 11461.15 & 11447.65 & 11474.65 & 26 & 8.093 & 0.084 & 0.897 & 0.611 & 0.027 & 0.007 & A \\
\hline 1999.85 & 11491.11 & 11475.65 & 11506.57 & 24 & 8.102 & 0.057 & 0.899 & 0.604 & 0.017 & 0.007 & A \\
\hline 2000.19 & 11613.48 & 11604.01 & 11622.95 & 14 & 8.079 & 0.140 & 0.903 & 0.599 & 0.052 & 0.008 & A \\
\hline 2000.28 & 11647.90 & 11628.94 & 11666.86 & 29 & 8.058 & 0.185 & 0.902 & 0.602 & 0.062 & 0.008 & $A+B$ \\
\hline 2000.38 & 11684.34 & 11667.91 & 11700.78 & 18 & 8.083 & 0.135 & 0.904 & 0.605 & 0.045 & 0.008 & A \\
\hline 2000.50 & 11726.22 & 11701.93 & 11750.52 & 22 & 8.096 & 0.123 & 0.894 & 0.617 & 0.033 & 0.009 & $A+B$ \\
\hline 2000.80 & 11839.10 & 11810.64 & 11867.57 & 25 & 8.083 & 0.147 & 0.906 & 0.597 & 0.053 & 0.008 & A \\
\hline 2001.20 & 11983.01 & 11958.04 & 12007.99 & 19 & 8.069 & 0.123 & 0.911 & 0.591 & 0.033 & 0.008 & A \\
\hline 2001.42 & 12062.21 & 12014.89 & 12109.53 & 43 & 8.055 & 0.170 & 0.906 & 0.606 & 0.054 & 0.008 & $A+B$ \\
\hline 2001.75 & 12184.01 & 12170.35 & 12197.67 & 22 & 8.083 & 0.140 & 0.909 & 0.602 & 0.046 & 0.008 & $A+B$ \\
\hline 2001.83 & 12214.62 & 12198.67 & 12230.58 & 17 & 8.110 & 0.111 & 0.898 & 0.608 & 0.037 & 0.008 & $A+B$ \\
\hline 2002.19 & 12345.49 & 12328.04 & 12362.93 & 17 & 8.039 & 0.130 & 0.897 & 0.584 & 0.041 & 0.008 & A \\
\hline 2002.27 & 12373.91 & 12365.92 & 12381.90 & 10 & 8.052 & 0.134 & 0.894 & 0.568 & 0.049 & 0.008 & A \\
\hline 2002.42 & 12427.39 & 12397.90 & 12456.88 & 26 & 8.077 & 0.076 & 0.897 & 0.565 & 0.023 & 0.012 & A \\
\hline 2002.81 & 12570.65 & 12537.74 & 12603.56 & 22 & 8.077 & 0.134 & 0.895 & 0.606 & 0.049 & 0.013 & A \\
\hline 2003.21 & 12714.98 & 12693.04 & 12736.92 & 15 & 8.055 & 0.102 & 0.896 & 0.573 & 0.031 & 0.016 & A \\
\hline 2003.43 & 12796.97 & 12772.97 & 12820.96 & 14 & 8.024 & 0.195 & 0.898 & 0.600 & 0.071 & - & A \\
\hline 2003.75 & 12913.66 & 12896.67 & 12930.65 & 20 & 8.067 & 0.107 & 0.893 & 0.610 & 0.033 & 0.009 & A \\
\hline 2003.85 & 12949.60 & 12932.64 & 12966.57 & 20 & 8.059 & 0.093 & 0.890 & 0.589 & 0.027 & 0.013 & A \\
\hline 2004.19 & 13075.48 & 13058.03 & 13092.93 & 10 & 8.051 & 0.117 & 0.886 & 0.549 & 0.043 & 0.013 & A \\
\hline 2004.32 & 13120.86 & 13102.89 & 13138.82 & 18 & 8.022 & 0.114 & 0.887 & 0.562 & 0.036 & 0.15 & A \\
\hline 2004.45 & 13170.37 & 13148.80 & 13191.95 & 13 & 8.022 & 0.125 & 0.891 & 0.584 & 0.040 & 0.013 & A \\
\hline 2004.92 & 13341.85 & 13255.69 & 13428.02 & 29 & 8.033 & 0.129 & 0.897 & 0.594 & 0.036 & 0.013 & A \\
\hline \multicolumn{12}{|c|}{ AR Lac } \\
\hline 1990.25 & 7982.74 & 7896.57 & 8068.92 & 21 & 6.105 & 0.624 & 0.771 & 0.311 & 0.186 & 0.010 & A \\
\hline 1990.86 & 8206.22 & 8143.88 & 8268.56 & 54 & 6.093 & 0.608 & 0.787 & 0.309 & 0.205 & 0.010 & A \\
\hline 1991.71 & 8517.76 & 8403.96 & 8631.57 & 66 & 6.084 & 0.648 & 0.781 & 0.327 & 0.218 & 0.010 & A \\
\hline 1992.67 & 8866.79 & 8759.98 & 8973.60 & 90 & 6.096 & 0.618 & 0.766 & 0.287 & 0.118 & 0.006 & $A+B$ \\
\hline 1993.71 & 9247.57 & 9139.56 & 9355.57 & 108 & 6.053 & 0.665 & 0.760 & 0.285 & 0.156 & 0.004 & $A+B$ \\
\hline 1994.65 & 9590.16 & 9518.97 & 9661.35 & 43 & 6.093 & 0.418 & 0.743 & 0.278 & 0.089 & 0.007 & $A+B$ \\
\hline 1995.57 & 9924.63 & 9877.87 & 9971.40 & 40 & 6.079 & 0.162 & 0.742 & 0.301 & 0.039 & 0.010 & A \\
\hline 1995.87 & 10036.20 & 9981.81 & 10090.58 & 42 & 6.073 & 0.391 & 0.754 & 0.278 & 0.057 & 0.010 & $\mathrm{~A}+\mathrm{B}$ \\
\hline 1996.74 & 10354.02 & 10254.47 & 10453.58 & 75 & 6.081 & 0.689 & 0.774 & 0.297 & 0.158 & 0.010 & $A+B$ \\
\hline 1997.40 & 10596.43 & 10557.00 & 10635.85 & 43 & 6.124 & 0.080 & 0.755 & 0.286 & 0.018 & 0.010 & $A+B$ \\
\hline 1997.59 & 10663.02 & 10644.40 & 10681.65 & 38 & 6.095 & 0.698 & 0.763 & 9.999 & 0.154 & 0.010 & $A+B$ \\
\hline 1997.84 & 10755.46 & 10689.36 & 10821.57 & 1057 & 6.107 & 0.705 & 0.803 & 0.385 & 0.233 & 0.010 & $A+B$ \\
\hline 1998.40 & 10961.44 & 10925.99 & 10996.89 & 41 & 6.120 & 0.645 & 0.761 & 0.294 & 0.155 & 0.010 & $A+B$ \\
\hline 1998.87 & 11133.19 & 11078.81 & 11187.57 & 41 & 6.111 & 0.647 & 0.758 & 0.301 & 0.136 & 0.010 & $A+B$ \\
\hline 1999.45 & 11342.26 & 11293.99 & 11390.54 & 63 & 6.095 & 0.634 & 0.786 & 0.330 & 0.222 & 0.010 & B \\
\hline 1999.81 & 11476.10 & 11401.63 & 11550.57 & 70 & 6.084 & 0.651 & 0.771 & 0.311 & 0.205 & 0.010 & B \\
\hline 2000.45 & 11709.31 & 11658.99 & 11759.62 & 50 & 6.119 & 0.644 & 0.766 & 0.332 & 0.223 & 0.010 & B \\
\hline 2000.84 & 11853.21 & 11805.80 & 11900.61 & 81 & 6.118 & 0.641 & 0.756 & 0.301 & 0.148 & 0.010 & A \\
\hline 2001.66 & 12151.78 & 12020.99 & 12282.57 & 97 & 6.090 & 0.635 & 0.769 & 0.294 & 0.182 & 0.010 & $A+B$ \\
\hline 2002.66 & 12517.28 & 12390.99 & 12643.58 & 91 & 6.100 & 0.660 & 0.768 & 0.280 & 0.184 & 0.010 & $A+B$ \\
\hline 2003.40 & 12785.43 & 12750.00 & 12820.87 & 19 & 6.171 & 0.651 & 0.806 & 0.341 & 0.270 & 0.010 & A \\
\hline 2003.85 & 12951.23 & 12894.88 & 13007.58 & 54 & 6.107 & 0.670 & 0.770 & 0.282 & 0.183 & 0.010 & A \\
\hline 2004.66 & 13247.28 & 13123.98 & 13370.59 & 64 & 6.116 & 0.533 & 0.770 & 0.284 & 0.118 & 0.011 & A \\
\hline
\end{tabular}


Table 4. continued.

\begin{tabular}{|c|c|c|c|c|c|c|c|c|c|c|c|}
\hline Mean epoch & $\mathrm{HJD}_{\text {mean }}$ & $\mathrm{HJD}_{\text {ini }}$ & $\mathrm{HJD}_{\text {end }}$ & $N_{\mathrm{m}}$ & $V_{\min }$ & $\overline{\Delta V}$ & $\overline{B-V}$ & $U-B$ & $\sigma_{v}$ & $\sigma_{c k 1-c}$ & Note \\
\hline \multicolumn{12}{|c|}{ SZ Psc } \\
\hline 1993.83 & 9289.87 & 9228.49 & 9351.25 & 19 & 7.316 & 0.118 & 0.839 & 0.350 & 0.035 & 0.010 & B \\
\hline 1994.74 & 9623.38 & 9541.51 & 9705.26 & 61 & 7.285 & 0.258 & 0.848 & 0.387 & 0.061 & 0.012 & B \\
\hline 1995.70 & 9974.43 & 9905.56 & 10043.29 & 22 & 7.270 & 0.158 & 0.838 & 0.354 & 0.049 & 0.021 & B \\
\hline 1996.53 & 10278.50 & 10252.57 & 10304.42 & 17 & 7.289 & 0.137 & 0.824 & 0.349 & 0.036 & 0.02 & B \\
\hline 1996.83 & 10385.89 & 10374.46 & 10397.31 & 7 & 7.299 & 0.129 & 0.851 & 0.332 & 0.052 & 0.011 & B \\
\hline 1997.83 & 10753.79 & 10702.34 & 10805.25 & 13 & 7.276 & 0.397 & 0.854 & 0.356 & 0.101 & 0.016 & B \\
\hline 1998.67 & 11061.44 & 11039.42 & 11083.47 & 24 & 7.271 & 0.276 & 0.850 & 0.356 & 0.076 & 0.011 & B \\
\hline 1998.93 & 11154.31 & 11121.36 & 11187.26 & 22 & 7.296 & 0.379 & 0.868 & 0.438 & 0.136 & 0.013 & B \\
\hline \multicolumn{12}{|c|}{ II Peg } \\
\hline 1992.04 & 8638.09 & 8623.60 & 8652.57 & 7 & 7.482 & 0.125 & 1.012 & 0.691 & 0.042 & 0.008 & A \\
\hline 1992.50 & 8806.43 & 8802.94 & 8809.92 & 6 & 7.537 & 0.093 & 1.021 & 0.683 & 0.045 & 0.009 & A \\
\hline 1992.72 & 8887.66 & 8846.52 & 8928.80 & 20 & 7.497 & 0.168 & 1.028 & 0.665 & 0.052 & 0.006 & $A+B$ \\
\hline 1992.90 & 8952.05 & 8948.39 & 8955.71 & 16 & 7.508 & 0.167 & 1.030 & 0.667 & 0.066 & 0.008 & A \\
\hline 1993.52 & 9178.44 & 9160.97 & 9195.92 & 8 & 7.437 & 0.230 & 1.014 & 0.642 & 0.077 & 0.005 & A \\
\hline 1993.76 & 9265.18 & 9250.87 & 9279.49 & 31 & 7.439 & 0.258 & 1.031 & 0.661 & 0.094 & 0.007 & $A+B$ \\
\hline 1993.94 & 9329.67 & 9299.74 & 9359.60 & 10 & 7.492 & 0.220 & 1.025 & 0.676 & 0.073 & 0.012 & A \\
\hline 1994.58 & 9564.07 & 9541.54 & 9586.59 & 15 & 7.439 & 0.260 & 1.028 & 1.391 & 0.099 & 0.010 & B \\
\hline 1994.77 & 9634.00 & 9607.47 & 9660.52 & 18 & 7.452 & 0.228 & 1.340 & 1.243 & 0.078 & 0.010 & B \\
\hline 1994.94 & 9695.85 & 9676.36 & 9715.34 & 14 & 7.479 & 0.185 & 1.026 & 1.064 & 0.067 & 0.009 & B \\
\hline 1995.49 & 9895.26 & 9878.93 & 9911.60 & 20 & 7.489 & 0.220 & 0.666 & 1.021 & 0.080 & 0.008 & $A+B$ \\
\hline 1995.76 & 9995.13 & 9981.82 & 10008.43 & 35 & 7.533 & 0.187 & 1.362 & 1.184 & 0.060 & 0.007 & $A+B$ \\
\hline 1995.89 & 10044.52 & 10014.72 & 10074.31 & 39 & 7.528 & 0.186 & 1.082 & 1.025 & 0.055 & 0.011 & $A+B$ \\
\hline 1996.49 & 10263.54 & 10247.56 & 10279.52 & 16 & 7.418 & 0.300 & 0.993 & 0.689 & 0.096 & 0.010 & B \\
\hline 1996.58 & 10294.53 & 10289.55 & 10299.50 & 9 & 7.435 & 0.228 & 1.051 & 0.659 & 0.074 & 0.009 & B \\
\hline 1996.61 & 10307.49 & 10299.50 & 10315.48 & 11 & 7.425 & 0.289 & 1.040 & 0.640 & 0.110 & 0.010 & B \\
\hline 1996.85 & 10395.61 & 10392.70 & 10398.51 & 20 & 7.432 & 0.333 & 1.029 & 0.660 & 0.112 & - & B \\
\hline 1996.91 & 10415.96 & 10401.65 & 10430.27 & 25 & 7.452 & 0.291 & 1.024 & 0.655 & 0.103 & 0.006 & $A+B$ \\
\hline 1996.99 & 10445.42 & 10436.23 & 10454.60 & 16 & 7.471 & 0.284 & 0.972 & 1.499 & 0.103 & 0.005 & A \\
\hline 1997.05 & 10469.59 & 10457.59 & 10481.58 & 11 & 7.477 & 0.284 & 1.024 & 0.640 & 0.111 & 0.009 & A \\
\hline 1997.46 & 10615.92 & 10596.96 & 10634.87 & 33 & 7.453 & 0.296 & 1.024 & 0.672 & 0.106 & 0.011 & A \\
\hline 1997.62 & 10673.99 & 10644.46 & 10703.53 & 37 & 7.418 & 0.331 & 1.049 & 0.635 & 0.104 & - & B \\
\hline 1997.74 & 10719.64 & 10714.78 & 10724.49 & 28 & 7.480 & 0.330 & 1.025 & 0.677 & 0.124 & - & B \\
\hline 1997.89 & 10772.89 & 10740.46 & 10805.33 & 38 & 7.476 & 0.337 & 1.021 & 0.674 & 0.119 & 0.007 & $A+B$ \\
\hline 1998.04 & 10828.59 & 10814.60 & 10842.59 & 11 & 7.473 & 0.263 & 1.001 & 0.669 & 0.089 & 0.005 & A \\
\hline 1998.46 & 10981.93 & 10966.95 & 10996.91 & 22 & 7.486 & 0.198 & 1.020 & 0.676 & 0.070 & 0.006 & A \\
\hline 1998.79 & 11103.14 & 11078.84 & 11127.44 & 51 & 7.494 & 0.259 & 1.028 & 0.667 & 0.084 & 0.007 & $A+B$ \\
\hline 1998.96 & 11165.15 & 11128.71 & 11201.59 & 50 & 7.445 & 0.348 & 1.023 & 0.662 & 0.112 & 0.005 & $A+B$ \\
\hline 1999.49 & 11358.80 & 11324.97 & 11392.62 & 23 & 7.470 & 0.463 & 1.041 & 0.686 & 0.145 & 0.009 & $A+B$ \\
\hline 1999.77 & 11462.31 & 11429.84 & 11494.77 & 43 & 7.410 & 0.485 & 1.024 & 0.663 & 0.164 & 0.005 & A \\
\hline 1999.97 & 11533.66 & 11495.74 & 11571.59 & 54 & 7.414 & 0.529 & 1.025 & 0.662 & 0.178 & 0.009 & $A+B$ \\
\hline 2000.45 & 11710.46 & 11689.97 & 11730.96 & 18 & 7.435 & 0.584 & 1.028 & 0.674 & 0.197 & 0.010 & A \\
\hline 2000.61 & 11767.59 & 11735.53 & 11799.64 & 19 & 7.497 & 0.317 & 1.035 & 0.677 & 0.110 & - & B \\
\hline 2000.89 & 11869.55 & 11803.51 & 11935.58 & 80 & 7.376 & 0.671 & 1.024 & 0.666 & 0.217 & 0.008 & $A+B$ \\
\hline 2001.43 & 12067.46 & 12055.97 & 12078.95 & 13 & 7.483 & 0.349 & 1.023 & 0.686 & 0.123 & 0.009 & A \\
\hline 2001.77 & 12191.62 & 12157.47 & 12225.78 & 49 & 7.536 & 0.270 & 1.032 & 0.675 & 0.087 & 0.006 & $A+B$ \\
\hline 2001.97 & 12264.68 & 12228.77 & 12300.59 & 37 & 7.571 & 0.205 & 1.024 & 0.674 & 0.062 & 0.010 & $A+B$ \\
\hline 2002.55 & 12474.77 & 12422.96 & 12526.57 & 24 & 7.445 & 0.348 & 1.029 & 0.656 & 0.107 & 0.014 & $A+B$ \\
\hline 2002.90 & 12604.73 & 12537.87 & 12671.58 & 77 & 7.396 & 0.391 & 1.016 & 0.662 & 0.128 & 0.010 & A \\
\hline 2003.45 & 12805.43 & 12783.97 & 12826.88 & 17 & 7.452 & 0.425 & 1.015 & 0.680 & 0.148 & 0.009 & A \\
\hline 2003.64 & 12873.90 & 12828.88 & 12918.91 & 30 & 7.482 & 0.321 & 1.024 & 0.667 & 0.104 & 0.009 & A \\
\hline 2003.85 & 12951.75 & 12923.80 & 12979.69 & 39 & 7.478 & 0.257 & 1.019 & 0.665 & 0.083 & 0.014 & A \\
\hline 2004.00 & 13006.12 & 12980.66 & 13031.58 & 22 & 7.525 & 0.202 & 1.016 & 0.640 & 0.059 & 0.011 & A \\
\hline 2004.45 & 13170.93 & 13149.97 & 13191.88 & 17 & 7.476 & 0.175 & 1.000 & 0.664 & 0.061 & 0.010 & A \\
\hline 2004.80 & 13296.78 & 13258.85 & 13334.70 & 55 & 6.981 & 0.661 & 1.015 & 0.666 & 0.108 & 0.010 & A \\
\hline \multicolumn{12}{|c|}{ BY Dra } \\
\hline 1990.37 & 8025.96 & 8005.99 & 8045.93 & 15 & 8.137 & 0.067 & 1.162 & - & 0.023 & - & A \\
\hline 1990.79 & 8180.14 & 8173.66 & 8186.63 & 10 & 8.210 & 0.076 & 1.180 & 1.035 & 0.027 & 0.006 & A \\
\hline 1990.86 & 8206.57 & 8201.59 & 8211.56 & 6 & 8.196 & 0.075 & 1.183 & - & 0.025 & 0.009 & A \\
\hline 1991.30 & 8366.48 & 8359.97 & 8372.99 & 11 & 8.221 & 0.047 & 1.178 & 1.045 & 0.015 & 0.008 & A \\
\hline 1991.38 & 8394.93 & 8387.90 & 8401.96 & 12 & 8.196 & 0.078 & 1.179 & 1.062 & 0.025 & 0.006 & A \\
\hline 1991.80 & 8550.12 & 8532.65 & 8567.59 & 17 & 8.173 & 0.061 & 1.174 & - & 0.022 & - & A \\
\hline 1992.32 & 8737.97 & 8718.97 & 8756.98 & 20 & 8.074 & 0.112 & 1.169 & 1.048 & 0.036 & 0.006 & A \\
\hline 1992.43 & 8779.45 & 8772.92 & 8785.97 & 13 & 8.075 & 0.076 & 1.164 & 1.056 & 0.027 & 0.006 & A \\
\hline 1992.49 & 8803.36 & 8797.81 & 8808.91 & 13 & 8.073 & 0.080 & 1.171 & 1.076 & 0.033 & 0.008 & A \\
\hline 1992.71 & 8881.92 & 8861.48 & 8902.36 & 18 & 8.053 & 0.049 & 1.174 & 1.044 & 0.014 & - & B \\
\hline
\end{tabular}


S. Messina: Patterns of color variations in close binary systems. I., Online Material $p 8$

Table 4. continued.

\begin{tabular}{|c|c|c|c|c|c|c|c|c|c|c|c|}
\hline Mean epoch & $\mathrm{HJD}_{\text {mean }}$ & $\mathrm{HJD}_{\text {ini }}$ & $\mathrm{HJD}_{\text {end }}$ & $N_{\mathrm{m}}$ & $V_{\min }$ & $\Delta V$ & $B-V$ & $U-B$ & $\sigma_{v}$ & $\sigma_{c k 1-c}$ & Note \\
\hline 1992.83 & 8927.96 & 8909.37 & 8946.55 & 17 & 8.057 & 0.051 & 1.167 & 1.041 & 0.018 & 0.013 & $A+B$ \\
\hline 1993.31 & 9101.42 & 9095.94 & 9106.91 & 8 & 8.003 & 0.098 & 1.167 & 1.049 & 0.035 & 0.010 & A \\
\hline 1993.37 & 9124.44 & 9107.91 & 9140.97 & 14 & 8.014 & 0.098 & 1.161 & 1.045 & 0.031 & 0.008 & A \\
\hline 1993.52 & 9176.20 & 9155.87 & 9196.54 & 23 & 8.027 & 0.050 & 1.160 & 1.062 & 0.015 & 0.009 & $A+B$ \\
\hline 1993.72 & 9252.67 & 9236.66 & 9268.68 & 14 & 8.045 & 0.030 & 1.172 & 1.035 & 0.010 & 0.007 & $A+B$ \\
\hline 1994.35 & 9480.92 & 9452.00 & 9509.84 & 9 & 8.092 & 0.044 & 1.163 & 1.003 & 0.013 & 0.009 & $A+B$ \\
\hline 1994.47 & 9524.80 & 9510.81 & 9538.80 & 13 & 8.083 & 0.040 & 1.169 & 1.028 & 0.013 & 0.007 & $A+B$ \\
\hline 1995.47 & 9887.95 & 9877.94 & 9897.95 & 17 & 8.094 & 0.052 & 1.172 & 1.079 & 0.015 & 0.008 & A \\
\hline 1995.51 & 9904.93 & 9899.94 & 9909.92 & 15 & 8.100 & 0.037 & 1.170 & 1.064 & 0.010 & 0.008 & A \\
\hline 1995.76 & 9994.14 & 9982.65 & 10005.64 & 16 & 8.102 & 0.049 & 1.169 & 1.053 & 0.013 & 0.008 & A \\
\hline 1995.83 & 10020.11 & 10006.64 & 10033.58 & 11 & 8.110 & 0.038 & 1.168 & 1.050 & 0.013 & 0.007 & A \\
\hline 1996.37 & 10217.45 & 10182.97 & 10251.93 & 23 & 8.119 & 0.048 & 1.163 & 1.031 & 0.014 & 0.008 & A \\
\hline 1997.14 & 10500.01 & 10482.06 & 10517.96 & 22 & 8.164 & 0.049 & 1.182 & 1.042 & 0.014 & 0.006 & A \\
\hline 1997.21 & 10526.46 & 10518.01 & 10534.91 & 16 & 8.169 & 0.057 & 1.184 & 1.055 & 0.019 & 0.009 & A \\
\hline 1997.27 & 10546.90 & 10535.91 & 10557.90 & 24 & 8.163 & 0.073 & 1.180 & 1.044 & 0.024 & 0.007 & A \\
\hline 1997.59 & 10663.03 & 10645.58 & 10680.49 & 17 & 8.174 & 0.043 & 1.173 & 1.036 & 0.012 & - & A \\
\hline 1998.22 & 10893.93 & 10868.00 & 10919.86 & 5 & 8.162 & 0.051 & 1.184 & 1.035 & 0.021 & 0.008 & $A+B$ \\
\hline 1998.58 & 11025.46 & 11000.59 & 11050.34 & 11 & 8.093 & 0.101 & 1.172 & 1.026 & 0.033 & - & B \\
\hline 1998.69 & 11067.30 & 11058.31 & 11076.30 & 11 & 8.094 & 0.096 & 1.192 & 0.990 & 0.029 & - & B \\
\hline 1999.40 & 11324.45 & 11282.50 & 11366.40 & 16 & 8.096 & 0.026 & 1.160 & 1.069 & 0.007 & - & B \\
\hline 2000.28 & 11645.87 & 11633.91 & 11657.84 & 9 & 8.090 & 0.044 & 1.165 & 1.065 & 0.013 & 0.010 & $A+B$ \\
\hline 2000.55 & 11743.53 & 11735.57 & 11751.48 & 10 & 8.062 & 0.029 & 1.183 & 1.017 & 0.010 & 0.007 & B \\
\hline 2000.70 & 11803.34 & 11781.45 & 11825.24 & 15 & 8.003 & 0.055 & 1.185 & 1.008 & 0.017 & 0.008 & B \\
\hline 2001.44 & 12070.36 & 12047.78 & 12092.94 & 26 & 8.084 & 0.037 & 1.164 & 1.045 & 0.011 & 0.006 & $A+B$ \\
\hline 2001.79 & 12199.10 & 12169.63 & 12228.57 & 30 & 8.108 & 0.075 & 1.167 & 1.035 & 0.022 & 0.008 & A \\
\hline 2002.39 & 12417.30 & 12395.82 & 12438.78 & 11 & 8.068 & 0.176 & 1.166 & 1.023 & 0.059 & 0.011 & A \\
\hline 2002.80 & 12566.14 & 12537.71 & 12594.57 & 19 & 8.107 & 0.084 & 1.166 & 1.031 & 0.023 & 0.012 & A \\
\hline 2003.24 & 12724.98 & 12694.00 & 12755.96 & 23 & 8.143 & 0.068 & 1.176 & 1.038 & 0.020 & 0.010 & A \\
\hline 2003.42 & 12794.77 & 12760.82 & 12828.73 & 46 & 8.139 & 0.079 & 1.177 & 1.038 & 0.024 & 0.019 & A \\
\hline 2003.79 & 12927.61 & 12895.65 & 12959.57 & 29 & 8.175 & 0.094 & 1.177 & 1.034 & 0.030 & 0.011 & A \\
\hline 2004.44 & 13166.85 & 13141.78 & 13191.91 & 19 & 8.222 & 0.057 & 1.173 & 1.103 & 0.014 & 0.012 & A \\
\hline 2004.77 & 13288.62 & 13258.66 & 13318.58 & 20 & 8.227 & 0.039 & 1.167 & 1.061 & 0.011 & 0.012 & A \\
\hline
\end{tabular}


Table 5. Summary of regression and correlation analyses: mean epoch of light curve, number of data points, slopes $b_{b v}, b_{u b}$ and $b_{u b v}$ along with correlation coefficients and significance levels.

\begin{tabular}{|c|c|c|c|c|c|c|c|c|c|c|c|}
\hline $\mathrm{HJD}_{\text {mean }} N_{V}$ & $b_{b v}$ & $r_{b v}$ & $\alpha$ & $N_{B V}$ & $b_{u b}$ & $r_{u b}$ & $\alpha$ & $N_{U B V}$ & $b_{u b v}$ & $r_{u b v}$ & $\alpha$ \\
\hline \multicolumn{12}{|c|}{ AR Psc } \\
\hline $7136.6258 \quad 13$ & 0.025 & 0.188 & $\alpha>0.2$ & 13 & 0.155 & 0.188 & $\alpha>0.2$ & 13 & 0.225 & 0.103 & $\alpha>0.2$ \\
\hline 7879.087213 & -0.077 & -0.351 & $\alpha>0.2$ & 13 & -0.128 & -0.351 & $\alpha>0.2$ & 13 & -0.641 & -0.547 & $0.1<\alpha<0.2$ \\
\hline 8195.350614 & -0.066 & -0.259 & $\alpha>0.2$ & 14 & -0.165 & -0.269 & $\alpha>0.2$ & 13 & -0.593 & -0.324 & $\alpha>0.2$ \\
\hline 8556.826511 & -0.332 & -0.722 & $\alpha<0.05$ & - & - & - & - & - & - & - & - \\
\hline 8621.692311 & 0.021 & 0.179 & $\alpha>0.2$ & 11 & -0.233 & 0.304 & $\alpha>0.2$ & 6 & 0.684 & 0.290 & $\alpha>0.2$ \\
\hline 8934.098420 & -0.016 & -0.059 & $\alpha>0.2$ & 20 & 0.028 & -0.059 & $\alpha>0.2$ & 20 & -0.327 & -0.132 & $\alpha>0.2$ \\
\hline 8994.981117 & 0.024 & 0.151 & $\alpha>0.2$ & 17 & 0.083 & 0.151 & $\alpha>0.2$ & 17 & 0.676 & 0.392 & $\alpha>0.2$ \\
\hline 9307.515428 & 0.018 & 0.064 & $\alpha>0.2$ & 28 & 0.055 & 0.064 & $\alpha>0.2$ & 28 & -0.206 & -0.269 & $0.1<\alpha<0.2$ \\
\hline 9593.043210 & -0.337 & -0.262 & $\alpha>0.2$ & 10 & -0.674 & -0.262 & $\alpha>0.2$ & 10 & -0.332 & -0.411 & $\alpha>0.2$ \\
\hline 9679.407611 & -0.175 & -0.454 & $\alpha>0.2$ & 11 & -0.101 & -0.454 & $\alpha>0.2$ & 11 & 0.057 & 0.029 & $\alpha>0.2$ \\
\hline $9898.4334 \quad 9$ & 0.352 & 0.363 & $\alpha>0.2$ & 9 & -0.355 & 0.224 & $\alpha>0.2$ & 7 & -1.163 & -0.672 & $0.05<\alpha<0.1$ \\
\hline 9995.796921 & -0.384 & -0.447 & $0.05<\alpha<0.1$ & 21 & 0.468 & -0.439 & $0.05<\alpha<0.1$ & 20 & -0.760 & -0.607 & $\alpha<0.05$ \\
\hline 10029.091214 & 0.055 & 0.073 & $\alpha>0.2$ & 14 & 0.079 & 0.003 & $\alpha>0.2$ & 13 & -0.522 & -0.752 & $\alpha<0.05$ \\
\hline 10388.096910 & -0.599 & -0.619 & $0.05<\alpha<0.1$ & 10 & -0.400 & -0.619 & $0.05<\alpha<0$ & 10 & 0.054 & 0.089 & $\alpha>0.2$ \\
\hline 10429.044817 & 0.020 & 0.023 & $\alpha>0.2$ & 17 & 0.072 & 0.023 & $\alpha>0.2$ & 17 & -0.707 & -0.652 & $\alpha<0.05$ \\
\hline 6714 & 0.120 & 0.2 & $\alpha>0$ & 14 & -0.042 & 0.217 & $\alpha>$ & 14 & -0.916 & -0 . & $\alpha<0.05$ \\
\hline 321 & -0.085 & -0.3 & $\alpha>c$ & 21 & 0.043 & -0.317 & $\alpha>$ & 21 & -0.101 & -0 & $\alpha>$ \\
\hline 616 & 0.032 & 0.0 & $\alpha>0.2$ & 16 & 0.093 & 0.090 & $\alpha>$ & 16 & 0.107 & 0.087 & $\alpha>0.2$ \\
\hline 725 & 0.056 & 0.0 & $\alpha>0.2$ & 25 & 0.407 & 0.072 & $\alpha>$ & 24 & 0.010 & 0.007 & $\alpha>0.2$ \\
\hline 10844.1 & -0.147 & -0.355 & $\alpha>0.2$ & 14 & -0.049 & -0.370 & $\alpha>0.2$ & 13 & -1.234 & -0.524 & $0.1<\alpha<0.2$ \\
\hline 11051.4 & - & - & - & - & - & - & - & - & - & - & - \\
\hline 11133.73 & -0.000 & -0.002 & $\alpha>0.2$ & 61 & -0.118 & -0.012 & $\alpha>0.2$ & 59 & -0.151 & -0.132 & $0.05<\alpha<$ \\
\hline $09.1079 \quad 15$ & 0.191 & 0.401 & $\alpha>0.2$ & 15 & -0.165 & 0.401 & $\alpha>0.2$ & 15 & -0.677 & -0.512 & $0.1<\alpha<0$ \\
\hline 5126 & -0.258 & -0.211 & $\alpha>0.2$ & 26 & -0.403 & -0.390 & $0.05<\alpha<0.1$ & 22 & -0.152 & -0 . & $\alpha>0.2$ \\
\hline 11529.700711 & -0.201 & -0.193 & $\alpha>0.2$ & 11 & 0.210 & -0.193 & $\alpha>0.2$ & 11 & -0.388 & -0 . & $\alpha>0.2$ \\
\hline 11567.14 & -0.233 & -0.463 & $0.05<\alpha<0.1$ & 19 & -0.172 & -0.539 & $\alpha<0.05$ & 17 & -0.343 & -0 . & $\alpha>0.2$ \\
\hline 11792.5 & -0.122 & -0.360 & $\alpha>0.2$ & 10 & -0.534 & -0.332 & $\alpha>0.2$ & 9 & -0.993 & -0 . & $\alpha>0.2$ \\
\hline 318 & -0.007 & -0.069 & $\alpha>0.2$ & 18 & -0.112 & 0.019 & $\alpha>0.2$ & 17 & 0.006 & & $\alpha>0.2$ \\
\hline 019 & -0.011 & -0.019 & $\alpha>0.2$ & 19 & 0.155 & 0.178 & $\alpha>$ & 17 & 0.282 & & $\alpha>0.2$ \\
\hline 112 & 0.033 & & $\alpha>0.2$ & 12 & 0.054 & 0.1 & $\alpha>$ & 12 & 0.684 & & $\alpha>0.2$ \\
\hline 815 & & -0. & $\alpha>0.2$ & 15 & -0.071 & -0.3 & $\alpha>$ & 15 & 0.341 & & $\alpha>$ \\
\hline & -0 & -0 & $\alpha>$ & 5 & -0.007 & -0.0 & $\alpha>$ & 14 & -0 & & $\alpha<0.05$ \\
\hline 517 & 014 & & & 17 & -0.118 & & $\alpha>$ & 17 & -0.003 & & $\alpha>0.2$ \\
\hline 326 & 066 & & $\alpha>0.2$ & 26 & -0.185 & 0.201 & $\alpha>$ & 25 & -0.004 & -0.003 & $\alpha>0.2$ \\
\hline 5019 & 0.010 & 0.076 & $\alpha>0.2$ & 19 & -0.030 & 0.076 & $\alpha>0.2$ & 19 & -0.164 & -0 . & $\alpha>$ \\
\hline \multicolumn{12}{|c|}{ VY Ari } \\
\hline 8545.899918 & 0.089 & 0.585 & $\alpha<0.05$ & 18 & -0.223 & 0.510 & $0.05<\alpha<$ & 7 & 1.083 & 0.222 & $\alpha>0.2$ \\
\hline 915 & 50 & & $\alpha<0.05$ & - & - & - & - & 一 & - & - & - \\
\hline 8662.134320 & 0.092 & $0.3 \xi$ & $0.1<\alpha<0.2$ & 20 & 0.354 & 0.383 & $0.1<\alpha<0.2$ & 14 & 0.803 & 0.409 & $0.1<\alpha<0.2$ \\
\hline 8919.855728 & 0.178 & 0.616 & $\alpha<0.05$ & 28 & 0.282 & 0.616 & $\alpha<0.05$ & 28 & 0.855 & 0.627 & $\alpha<0.05$ \\
\hline $357 \quad 17$ & 0.150 & & $\alpha<0.05$ & 17 & 0.396 & & $\alpha<0.0$ & 17 & 1.196 & 0.468 & $0.1<\alpha<0.2$ \\
\hline 42615 & 0.209 & & $\alpha<0.05$ & 15 & 0.466 & 0.5 & $\alpha<0.05$ & 15 & 1.739 & 0.751 & $\alpha<0.05$ \\
\hline 21820 & 0.097 & 0.360 & $0.1<\alpha<0.2$ & 20 & 0.045 & 0.360 & $0.1<\alpha<0.2$ & 20 & 0.888 & 0.602 & $\alpha<0.05$ \\
\hline 79723 & 0.179 & 0.792 & $\alpha<0.05$ & 23 & 0.194 & & $\alpha<0.05$ & 22 & -0.172 & -0.067 & $\alpha>0.2$ \\
\hline 2922 & 0.134 & & $\alpha<0.05$ & 22 & 0.276 & & $\alpha<0$ & 20 & 0.897 & 01 & $\alpha<0.05$ \\
\hline 8328 & 0.160 & & $\alpha<0$ & 28 & 0.304 & & $\alpha<0$ & 28 & 0.761 & 0.527 & $\alpha<0.05$ \\
\hline 2812 & & & $\alpha<0$ & 12 & 0.297 & & $\alpha<0$ & 12 & 0.513 & 0.369 & $\alpha>0.2$ \\
\hline 617 & 0.133 & & $\alpha<0.05$ & 17 & & & $\alpha<0$ & 17 & 1.281 & 0.503 & $0.05<\alpha<0$ \\
\hline 923 & & & $0.1<\alpha$ & 23 & 308 & & $\alpha<0$ & 0 & 0.591 & 34 & $0.05<\alpha<0$ \\
\hline 822 & & & $<\alpha$ & 22 & 0.053 & & $0.05<a$ & 14 & 0.044 & 0.064 & $\alpha>0.2$ \\
\hline 2625 & & & $05<\alpha<0$ & 25 & 0.685 & & $\alpha<0.05$ & 24 & 0.625 & 0.379 & $0.05<\alpha<0$ \\
\hline 8216 & 0.032 & 0.1 & $\alpha>0.2$ & 16 & 0.338 & 0.105 & $\alpha>0$. & 16 & 0.106 & 0.055 & $\alpha>0.2$ \\
\hline 8718 & 0.298 & 0.613 & $\alpha<0.05$ & 18 & 0.244 & 0.613 & $\alpha<0.05$ & 18 & 0.410 & 0.420 & $0.1<\alpha<0.2$ \\
\hline 614 & 135 & & $\alpha>0$ & 14 & 0.721 & & $\alpha>$ & 14 & -0.021 & -0 & $\alpha>0.2$ \\
\hline 10839.330618 & -0.043 & -0.132 & $\alpha>0.2$ & 18 & 0.106 & -0.132 & $\alpha>0.2$ & 18 & 1.064 & 0.388 & $0.1<\alpha<0.2$ \\
\hline $10873.2576 \quad 7$ & 0.242 & 0.636 & $0.1<\alpha<0.2$ & 7 & 0.672 & 0.636 & $0.1<\alpha<0.2$ & 7 & 1.081 & 0.385 & $\alpha>0.2$ \\
\hline 11070.096221 & 0.347 & 0.423 & $0.05<\alpha<0.1$ & 21 & 0.314 & 0.423 & $0.05<\alpha<0.1$ & 21 & -0.046 & -0.044 & $\alpha>0.2$ \\
\hline 11144.969010 & 0.052 & 0.337 & $\alpha>0.2$ & 10 & 0.375 & 0.337 & $\alpha>0.2$ & 10 & -0.248 & -0.072 & $\alpha>0.2$ \\
\hline $11194.4158 \quad 12$ & 0.093 & 0.440 & $\alpha>0.2$ & 12 & 0.037 & 0.440 & $\alpha>0.2$ & 12 & -0.061 & -0.032 & $\alpha>0.2$ \\
\hline $11443.1494 \quad 4$ & - & - & - & - & - & - & - & - & - & - & - \\
\hline $11518.0303 \quad 6$ & 0.023 & & $\alpha>0.2$ & 6 & 0.207 & & & 6 & 0.676 & 0.262 & \\
\hline 11817.966857 & 0.125 & 0.854 & $\alpha<0.05$ & 57 & 0.181 & 0.854 & $\alpha<0.05$ & 57 & 1.075 & 0.781 & $\alpha<0.05$ \\
\hline 11885.702625 & 0.130 & 0.928 & $\alpha<0.05$ & 25 & 0.183 & 0.932 & $\alpha<0.05$ & 24 & 1.256 & 0.893 & $\alpha<0.05$ \\
\hline
\end{tabular}


Table 5. continued.

\begin{tabular}{|c|c|c|c|c|c|c|c|c|c|c|c|}
\hline $\mathrm{HJD}_{\text {mean }} N_{V}$ & $b_{b v}$ & $r_{b v}$ & $\alpha$ & $N_{B V}$ & $b_{u b}$ & $r_{u b}$ & $\alpha$ & $N_{U B V}$ & $b_{u b v}$ & $r_{u b v}$ & $\alpha$ \\
\hline 11934.125834 & 0.139 & 0.864 & $\alpha<0.05$ & 34 & 0.219 & 0.913 & $\alpha<0.05$ & 31 & 1.545 & 0.895 & $\alpha<0.05$ \\
\hline 12182.925431 & 0.162 & 0.630 & $\alpha<0.05$ & 31 & 0.228 & 0.631 & $\alpha<0.05$ & 30 & -0.159 & -0.098 & $\alpha>0.2$ \\
\hline 12220.229425 & 0.101 & 0.330 & $0.1<\alpha<0.2$ & 25 & 0.294 & 0.395 & $0.05<\alpha<0.1$ & 24 & 0.097 & 0.080 & $\alpha>0.2$ \\
\hline $12256.2544 \quad 19$ & 0.267 & 0.783 & $\alpha<0.05$ & 19 & 0.165 & 0.783 & $\alpha<0.05$ & 19 & 0.273 & 0.243 & $\alpha>0.2$ \\
\hline 12296.662419 & 0.062 & 0.145 & $\alpha>0.2$ & 19 & 0.455 & 0.145 & $\alpha>0.2$ & 19 & -0.416 & -0.232 & $\alpha>0.2$ \\
\hline 12556.366229 & 0.024 & 0.063 & $\alpha>0.2$ & 29 & 0.126 & 0.063 & $\alpha>0.2$ & 29 & 0.568 & 0.311 & $0.1<\alpha<0.2$ \\
\hline 12608.739029 & 0.156 & 0.658 & $\alpha<0.05$ & 29 & -0.096 & 0.654 & $\alpha<0.05$ & 28 & -0.287 & -0.195 & $\alpha>0.2$ \\
\hline 12652.6 & 0.184 & 0.570 & $\alpha<0.05$ & 28 & 0.265 & 0.570 & $\alpha<0.05$ & 28 & 0.244 & 0.140 & $\alpha>0.2$ \\
\hline 12917.353660 & 0.088 & 0.274 & $\alpha<0.05$ & 60 & 0.136 & 0.280 & $\alpha<0.05$ & 59 & 0.895 & 0.482 & $\alpha<0.05$ \\
\hline 12971.778443 & 0.131 & 0.289 & $\alpha<0.05$ & 43 & 0.072 & 0.428 & $\alpha<0.05$ & 42 & 0.424 & 0.279 & $\alpha<0.05$ \\
\hline 13026.631615 & -0.040 & -0.064 & $\alpha>0.2$ & 15 & 0.188 & -0.064 & $\alpha>0.2$ & 15 & 0.199 & 0.197 & $\alpha>0.2$ \\
\hline 13273.385336 & 0.038 & 0.190 & $0.1<\alpha<0.2$ & 36 & 0.173 & 0.303 & $\alpha<0.05$ & 34 & 0.114 & 0.083 & $\alpha>0.2$ \\
\hline 13348.710885 & 0.134 & 0.564 & $\alpha<0.05$ & 85 & 0.306 & 0.564 & $\alpha<0.05$ & 85 & 0.744 & 0.468 & $\alpha<0.05$ \\
\hline \multicolumn{12}{|c|}{ UX Ari } \\
\hline 01611 & -0.093 & -0.629 & $05<\alpha<0.1$ & 11 & -0.238 & -0.610 & $05<a$ & 8 & -0.628 & -0.207 & $\alpha>0.2$ \\
\hline 7948.6 & -0.104 & -0.343 & $\alpha>0.2$ & 9 & -0.612 & -0.713 & $\alpha<0.05$ & 6 & 1.667 & 0.697 & $\alpha<0.05$ \\
\hline 8188.939025 & -0.043 & -0.086 & $\alpha>0.2$ & 25 & -0.145 & -0.603 & $\alpha<0$ & 18 & 0.194 & 0.113 & $\alpha>0.2$ \\
\hline 8262.259017 & 0.167 & 0.323 & $\alpha>0.2$ & 17 & 0.031 & 0.379 & $\alpha>0.2$ & 16 & -0.158 & -0.082 & $\alpha>0.2$ \\
\hline 8555.409122 & -0.332 & -0.504 & $0.05<\alpha<0.1$ & - & - & - & - & - & - & - & - \\
\hline 1316 & -0.416 & -0.665 & $\alpha<0.05$ & 16 & -0.209 & -0.631 & $\alpha<0.05$ & 12 & -0.628 & -0.513 & $0.05<\alpha<0$ \\
\hline 529 & -0.122 & -0.480 & $\alpha<0$ & 29 & -0.279 & -0.450 & $\alpha<$ & 26 & 0.354 & 0.272 & $0.1<\alpha<0.2$ \\
\hline 126 & -0.126 & -0.857 & $\alpha<0.05$ & 26 & -0.247 & -0.852 & $\alpha<0$ & 25 & 1.514 & 0.625 & $\alpha<0.05$ \\
\hline 27519 & -0.128 & -0.813 & $\alpha<0.05$ & 19 & -0.316 & -0.829 & $\alpha<0.05$ & 13 & 1.513 & 0.674 & $\alpha<0.05$ \\
\hline 1651 & -0.113 & -0.750 & $\alpha<0.05$ & 51 & -0.291 & -0.746 & $\alpha<0.05$ & 48 & 1.424 & 0.582 & $\alpha<0.05$ \\
\hline 9386.014910 & -0.170 & -0.670 & $0.05<\alpha<0.1$ & 10 & -0.183 & -0.321 & $\alpha>0.2$ & 9 & -0.387 & -0.197 & $\alpha>0.2$ \\
\hline 9655.573227 & -0.142 & -0.891 & $\alpha<0.05$ & 27 & -0.317 & -0.891 & $\alpha<0.05$ & 27 & 1.741 & 0.841 & $\alpha<0.05$ \\
\hline 58315 & -0.155 & -0.723 & $\alpha<0.05$ & 15 & -0.287 & -0.752 & $\alpha<0.05$ & 14 & 0.679 & 0.400 & $\alpha>0.2$ \\
\hline 52838 & -0.155 & -0.860 & $\alpha<0.05$ & 38 & -0.319 & -0.861 & $\alpha<0.05$ & 36 & 1.425 & 0.685 & $\alpha<0.05$ \\
\hline 613 & -0.212 & -0.795 & $\alpha<0$ & 13 & -0.224 & -0.851 & $\alpha<0$ & 11 & 1.121 & 0.682 & $\alpha<0.05$ \\
\hline 937 & -0.161 & -0.818 & $\alpha<0$ & 37 & -0.347 & -0.8 & $\alpha<($ & 37 & 1.158 & & $\alpha<0.05$ \\
\hline 231 & -0.159 & -0.868 & $\alpha<0$ & 31 & -0.280 & -0.868 & $\alpha<0$ & 31 & 1.174 & 0.636 & $\alpha<0.05$ \\
\hline 134 & -0.178 & -0.659 & $\alpha<0$ & 34 & -0.295 & -0.659 & $\alpha<0$ & 34 & 0.251 & 0.187 & $\alpha>0.2$ \\
\hline 910 & .163 & -0.836 & $\alpha<0$ & 10 & -0.319 & -0.836 & $\alpha<$ & 10 & 1.195 & 0.661 & $0.05<\alpha<0$ \\
\hline 828 & 197 & -0.855 & $\alpha<$ & 28 & -0.276 & -0.8 & $\alpha<$ & 26 & 0.957 & 0.728 & $\alpha<0.05$ \\
\hline 149 & 158 & -0.777 & $\alpha<$ & 49 & 0.280 & -0.7 & $\alpha<$ & 47 & 1.229 & & 0.05 \\
\hline 7774 & -0.148 & -0.627 & $\alpha<$ & 74 & -0.210 & -0.629 & $\alpha<0$ & 73 & 0.443 & & $\alpha<0.05$ \\
\hline 9053 & -0.129 & -0.393 & $\alpha<$ & 53 & -0.204 & -0.393 & $\alpha<0$ & 53 & 0.307 & 0.288 & $\alpha<0.05$ \\
\hline 30127 & -0.189 & -0.751 & $\alpha<0.05$ & 27 & -0.330 & -0.751 & $\alpha<0.05$ & 27 & 0.908 & 0.509 & $\alpha<0.05$ \\
\hline 37034 & -0.141 & -0.465 & $\alpha<0.05$ & 34 & -0.343 & -0.464 & $\alpha<0.05$ & 33 & 0.289 & 0.193 & $\alpha>0.2$ \\
\hline 0.687837 & -0.154 & -0.562 & $\alpha<0.05$ & 37 & -0.279 & -0.562 & $\alpha<0.05$ & 37 & 0.874 & 0.520 & $\alpha<0.05$ \\
\hline 35915 & -0.099 & -0.419 & $\alpha>0.2$ & 15 & -0.110 & -0.419 & $\alpha>0.2$ & 15 & 0.328 & 0.272 & $\alpha>0.2$ \\
\hline 7695 & -0.227 & -0.582 & $\alpha<0.05$ & 95 & -0.214 & -0.582 & $\alpha<0.05$ & 95 & 0.090 & 0.062 & $\alpha>0.2$ \\
\hline 440 & -0.097 & -0.173 & $0.1<\alpha<0.2$ & 40 & -0.215 & -0.173 & $0.1<\alpha<0$. & 40 & 0.114 & 0.079 & $\alpha>0.2$ \\
\hline 8922 & -0.368 & -0.299 & $\alpha>0.2$ & 22 & .874 & -0.299 & $\alpha>$ & 22 & 0.209 & 100 & $>0.2$ \\
\hline & -0.289 & -0.433 & $0.1<\alpha<0.2$ & 19 & 0.441 & -0.433 & $0.1<\alpha$ & 19 & -0.548 & -0.206 & $\alpha>0.2$ \\
\hline 12 & -0.445 & -0.785 & $\alpha<0$ & 15 & -0.481 & -0.785 & $\alpha<0$ & 15 & 0.960 & 0.582 & $0.05<\alpha<0$ \\
\hline 953 & 201 & -0.790 & $\alpha<$ & 53 & -0.254 & -0.777 & $\alpha<$ & 52 & 0.817 & 96 & $\alpha<0.05$ \\
\hline 419 & & -0.868 & & 19 & .259 & -0 & & 19 & 0.661 & 22 & 0.05 \\
\hline 934 & & & & 34 & -0 & & & 33 & & & $\alpha<$ \\
\hline 735 & -0.198 & -0.861 & & 35 & -0.399 & -0 . & $\alpha<$ & 33 & 1.440 & 80 & $\alpha<0.05$ \\
\hline 13348.256468 & -0.205 & -0.931 & $\alpha<0.05$ & 68 & -0.360 & -0.931 & $\alpha<0.05$ & 68 & 1.553 & 0.876 & $\alpha<0.05$ \\
\hline \multicolumn{12}{|c|}{ V711 Tau } \\
\hline 223 & & -0.071 & & 23 & -0.223 & & $\alpha>$ & 23 & 408 & & $\alpha>0.2$ \\
\hline 4115 & .052 & -0.352 & $\alpha>$ & 15 & -0.249 & -0.352 & .2 & 15 & 0.888 & 0.246 & $>0.2$ \\
\hline 90410 & -0.115 & -0.459 & $\alpha>0.2$ & 10 & -0.330 & -0.459 & $>0.2$ & 10 & 0.489 & 0.238 & $>0.2$ \\
\hline 4716 & -0.031 & -0.109 & $\alpha>0.2$ & 16 & -0.192 & -0.599 & $\alpha<0.05$ & 13 & 0.757 & 0.324 & $>0.2$ \\
\hline 0.389013 & 0.067 & 0.228 & $\alpha>0.2$ & 13 & 0.149 & -0.342 & $\alpha>0.2$ & 10 & -1.818 & -0.452 & $>0.2$ \\
\hline 50314 & -0.151 & -0.478 & $0.1<\alpha<0.2$ & - & - & - & - & - & - & - & - \\
\hline $8614.7424 \quad 9$ & -0.047 & -0.216 & $\alpha>0.2$ & - & - & - & - & - & — & - & - \\
\hline 8661.139414 & -0.222 & -0.487 & $0.1<\alpha<0.2$ & 14 & -0.383 & -0.364 & $\alpha>0.2$ & 12 & 0.254 & 0.166 & $\alpha>0.2$ \\
\hline 9278.411224 & 0.104 & 0.501 & $\alpha<0.05$ & 24 & -0.065 & 0.501 & $\alpha<0.05$ & 24 & -0.148 & -0.145 & $\alpha>0.2$ \\
\hline 9315.621824 & 0.008 & 0.048 & $\alpha>0.2$ & 24 & -0.095 & -0.117 & $\alpha>0.2$ & 23 & -0.341 & -0.228 & $\alpha>0.2$ \\
\hline 9386.644029 & -0.157 & -0.588 & $\alpha<0.05$ & 29 & -0.236 & -0.588 & $\alpha<0.05$ & 29 & 0.632 & 0.463 & $\alpha<0.05$ \\
\hline 9996.911827 & -0.020 & -0.090 & $\alpha>0.2$ & 27 & -0.114 & -0.090 & $\alpha>0.2$ & 27 & -0.300 & -0.253 & $\alpha>0.2$ \\
\hline 10110.660017 & -0.084 & -0.225 & $\alpha>0.2$ & 17 & -0.020 & -0.219 & $\alpha>0.2$ & 16 & -0.121 & -0.083 & $\alpha>0.2$ \\
\hline
\end{tabular}


Table 5. continued.

\begin{tabular}{|c|c|c|c|c|c|c|c|c|c|c|c|}
\hline $\mathrm{HJD}_{\text {mean }} N_{V}$ & $b_{b v}$ & $r_{b v}$ & $\alpha$ & $N_{B V}$ & $b_{u b}$ & $r_{u b}$ & $\alpha$ & $N_{U B V}$ & $b_{u b v}$ & $r_{u b v}$ & $\alpha$ \\
\hline 10415.795650 & -0.005 & -0.032 & $\alpha>0.2$ & 50 & -0.193 & -0.079 & $\alpha>0.2$ & 48 & -0.302 & -0.149 & $0.05<\alpha<0.1$ \\
\hline $71.1731 \quad 39$ & -0.115 & -0.545 & $\alpha<0.05$ & 39 & -0.171 & -0.5 & $\alpha<0.05$ & 39 & 0.267 & 0.211 & $0.1<\alpha<0.2$ \\
\hline 8533 & -0.144 & -0.318 & $0.05<\alpha<0.1$ & 33 & -0.217 & -0.318 & $0.05<\alpha<0.1$ & 32 & -0.417 & -0.315 & $0.05<\alpha<0.1$ \\
\hline 10779.325928 & 0.080 & 0.192 & $\alpha>0.2$ & 28 & -0.046 & 0.234 & $\alpha>0.2$ & 27 & -0.134 & -0.098 & $\alpha>0.2$ \\
\hline 10811.225119 & -0.084 & -0.151 & $\alpha>0.2$ & 19 & -0.181 & -0.151 & $\alpha>0.2$ & 19 & -0.379 & -0.340 & $\alpha>0.2$ \\
\hline $10882.6177 \quad 11$ & -0.043 & -0.112 & $\alpha>0.2$ & 11 & 0.219 & -0.199 & $\alpha>0.2$ & 10 & -0.952 & -0.447 & $\alpha>0.2$ \\
\hline 11085.587635 & 0.003 & 0.010 & $\alpha>0.2$ & 35 & 0.045 & -0.275 & $0.05<\alpha<0.1$ & 18 & 0.122 & 0.114 & $\alpha>0.2$ \\
\hline 9.178331 & -0.017 & -0.092 & $\alpha>0.2$ & 31 & 0.034 & -0.177 & $\alpha>0.2$ & 24 & -0.281 & -0.280 & $0.1<\alpha<0.2$ \\
\hline 8326 & -0.076 & -0.2 & $\alpha>0.2$ & 26 & -0.090 & -0.239 & $\alpha>0.2$ & 26 & -0.427 & -0.399 & $0.05<\alpha<0.1$ \\
\hline 847 & -0.080 & -0.3 & $\alpha<0.05$ & 47 & -0.180 & -0.377 & $\alpha<0.05$ & 47 & -0.063 & -0.039 & $\alpha>0.2$ \\
\hline 711 & -0.074 & -0.2 & $\alpha>0.2$ & 11 & -0.232 & -0.510 & $0.1<\alpha<0.2$ & 10 & 0.564 & 0.376 & $\alpha>0.2$ \\
\hline 639 & -0.064 & -0.2 & $\alpha<0.05$ & 39 & -0.107 & -0. & $\alpha<0.05$ & 39 & -0.253 & -0.222 & $0.05<\alpha<0.1$ \\
\hline 119 & -0.011 & -0.0 & $\alpha>0.2$ & 29 & -0.180 & & $\alpha>0.2$ & 29 & -0.138 & -0.073 & $\alpha>0.2$ \\
\hline 6531 & -0.046 & -0.2 & $\alpha>0.2$ & 31 & 0.050 & -0.2 & $0.1<\alpha<0.2$ & 30 & -0.409 & -0.336 & $0.05<$ \\
\hline 826 & 0.005 & & $\alpha>0.2$ & 6 & -0.290 & & & 6 & 1.200 & & 0.2 \\
\hline 221 & -0.155 & -0.3 & $\alpha>0.2$ & 21 & -0.286 & -0 & $\alpha>$ & 21 & -0.403 & -0 . & 0.2 \\
\hline & 0.210 & 0.689 & $\alpha<0.05$ & 14 & -0.461 & & $\alpha<0.05$ & 14 & -1.693 & -0.725 & $\alpha<0.05$ \\
\hline 3.362728 & -0.103 & -0.362 & $.05<\alpha<0.1$ & 28 & -0.098 & -0. & $0.05<\alpha<0.1$ & 28 & 0.471 & 0.262 & $0.1<\alpha<0$ \\
\hline \multicolumn{12}{|c|}{$\begin{array}{l}\text { EI Eri } \\
\end{array}$} \\
\hline 7136.726713 & 0.283 & 0.749 & $\alpha<0.05$ & 13 & 0.121 & 0.74 & $\alpha<0.05$ & 13 & -0.104 & -0.059 & $\alpha>0.2$ \\
\hline $7879.2407 \quad 10$ & 0.251 & 0.889 & $\alpha<0.05$ & 10 & 0.189 & & $\alpha<0.05$ & 0 & 0.337 & 0.247 & 0.2 \\
\hline 8199.417920 & 0.013 & 0.053 & $\alpha>0.2$ & 20 & 0.138 & 0.076 & $\alpha>0.2$ & 19 & 0.366 & 0.175 & $\alpha>0.2$ \\
\hline 8297.172115 & -0.367 & -0.520 & $05<\alpha<0.1$ & 15 & 0.884 & -0.526 & $0.05<\alpha<0.1$ & 13 & -0.994 & -0.494 & $0.1<\alpha<0.2$ \\
\hline $55.3957 \quad 17$ & -0.038 & -0.162 & $\alpha>0.2$ & - & - & - & - & - & - & - & - \\
\hline 2.166015 & 0.058 & 0.070 & $\alpha>0.2$ & 15 & 521 & & $0.1<\alpha<0$. & 8 & -0.297 & -0.157 & $\alpha>0.2$ \\
\hline 14.395311 & 0.115 & 0.6 & $05<\alpha<0$ & 11 & -0.173 & & $\alpha<0.05$ & 8 & .475 & & $\alpha>0.2$ \\
\hline 00.702623 & -0.019 & -0.0 & $\alpha>0.2$ & 23 & 0.123 & & & 20 & & -0 . & $\alpha>0.2$ \\
\hline 4.969314 & 0.123 & & $1<\alpha<0$ & 14 & 0.1 & & $0.1<\alpha<0$ & 13 & & -0.072 & \\
\hline 93 & 0.125 & 0.2 & & 60 & 0.157 & & & 59 & 0.123 & 0.111 & $0.05<\alpha<0.1$ \\
\hline 322 & 0.217 & & 05 & 22 & 0.0 & & 05 & 17 & & & \\
\hline 111 & -0.058 & -0.133 & $>0.2$ & 11 & 0.166 & & & 11 & -0 & -0 . & 0.2 \\
\hline 440 & 0.133 & & $05<\alpha<0$ & 40 & 0.466 & & & 29 & -0.078 & -0.046 & $\alpha>0.2$ \\
\hline 922 & 0.241 & 0. & $<<$ & 22 & 0.214 & & $\alpha<$ & 22 & 0.429 & 0.430 & $0.05<\alpha<0$ \\
\hline 12.916826 & 0.256 & 0.4 & $\alpha<0.05$ & 26 & 0.181 & & $\alpha<0$ & 23 & -0.010 & -0.007 & $\alpha>0.2$ \\
\hline $52.8004 \quad 16$ & 0.005 & 0.010 & $\alpha>0.2$ & 16 & -0.006 & & $\alpha>0.2$ & 13 & -0.252 & -0.160 & $\alpha>0.2$ \\
\hline 10495.692424 & 0.139 & 0.203 & $\alpha>0.2$ & 24 & 0.423 & 0.354 & $0.1<\alpha<0$. & 22 & -0.121 & -0.075 & $\alpha>0.2$ \\
\hline 730.910424 & 0.193 & 196 & $\alpha>0.2$ & 24 & 0.571 & & $\alpha>0.2$ & 23 & -0.494 & -0.242 & $\alpha>0.2$ \\
\hline 6.851240 & 0.079 & & $\alpha>$ & 40 & 0.300 & & $0.1<\alpha$ & 38 & -0.199 & -0.103 & $\alpha>$ \\
\hline 8026 & -0.122 & -0.212 & $\alpha>0.2$ & 26 & 0.257 & -0 & & 25 & 1.471 & & $\alpha<0.05$ \\
\hline 19615 & 305 & & $\alpha<0$ & 15 & & & $\alpha$ & 15 & & & \\
\hline & & & & 20 & 0.037 & & & 18 & 0.069 & -0 . & $\alpha>0.2$ \\
\hline $7414 \quad 8$ & & & $\alpha<$ & 8 & -0.048 & & $\alpha<$ & 8 & 91 & -0 . & 0.2 \\
\hline 1911 & & & & 11 & & & & 11 & & & 0.2 \\
\hline & & & & 24 & -0.5 & & & 22 & & & \\
\hline 920 & & & & 0 & & & & 8 & & -0 . & 0.05 \\
\hline 612 & 0.056 & & & 12 & 311 & & & 11 & & -0 . & 0.0 \\
\hline 8.772923 & & & $\alpha<$ & 23 & -0.044 & & $\alpha<$ & 21 & -0.629 & -0 . & $0.1<\alpha<0.2$ \\
\hline $49.4890 \quad 17$ & 0.050 & & 0.2 & 17 & 0.529 & & $\alpha>$ & 17 & -0.448 & -0.166 & $\alpha>0.2$ \\
\hline 207.023440 & 0.024 & & $\alpha>0.2$ & 40 & 0.014 & & $\alpha>0.2$ & 40 & -0.187 & -0.058 & $\alpha>0.2$ \\
\hline 12269.769913 & 0.123 & & $\alpha>0.2$ & 13 & -0.140 & & $0.1<\alpha<0$. & 11 & -0.938 & -0.550 & $0.05<\alpha<0.1$ \\
\hline 2.659013 & 0.296 & & $\alpha>0.2$ & 13 & 0.253 & 0.406 & $\alpha>0.2$ & 12 & 0.659 & 0.500 & $0.1<\alpha<0.2$ \\
\hline $5.8647 \quad 14$ & 0.095 & & $\alpha>$ & & 0.306 & & $0.05<c$ & 12 & 1.482 & 0.781 & $\alpha<0.05$ \\
\hline 9.253222 & -0.072 & -0.213 & $\alpha>0.2$ & 22 & 0.282 & -0.213 & $\alpha>0.2$ & 22 & -0.769 & -0.388 & $0.1<\alpha<0.2$ \\
\hline & & & $\alpha>$ & 1 & -0.1 & & 0 & & & & \\
\hline 923928 & -0.258 & -0.334 & $0.05<\alpha<0.1$ & 28 & -0.348 & -0.3 & $0.1<\alpha$ & 23 & 0.096 & 0.058 & $\alpha>0.2$ \\
\hline 933 & -0.276 & -0.5 & $\alpha<0.05$ & 33 & & & $\alpha<0.05$ & 31 & -0.463 & -0.322 & $0.05<\alpha<0.1$ \\
\hline 122 & -0.188 & -0.2 & & 22 & -0.1 & & & 20 & -0. & -0.233 & $\alpha>0.2$ \\
\hline $028.1535 \quad 17$ & & & & 17 & & & & 16 & & & 0.2 \\
\hline & -0.3 & -0.5 & & 8 & -0.0 & -0.6 & $0.05<c$ & 7 & & & \\
\hline 632 & & & & 32 & & & & 25 & -0 . & -0 . & \\
\hline 330 & & & & 30 & & & & 26 & & 0.290 & $0.1<\alpha<0.2$ \\
\hline 13398.650742 & 0.091 & 0.239 & $0.05<\alpha<0.1$ & 42 & 0.182 & 0.244 & $\alpha<0.05$ & 39 & 0.055 & 0.039 & \\
\hline & & & & & & & & & & & \\
\hline & & & & 1 & & & & & -0 . & -0 & \\
\hline & & & & & & & & & & & \\
\hline 8312.183514 & 0.072 & 0.292 & $\alpha>0.2$ & 14 & 0.489 & 0.292 & $\alpha>0.2$ & 14 & 1.346 & 0.480 & $0.1<\alpha<0.2$ \\
\hline
\end{tabular}


Table 5. continued.

\begin{tabular}{|c|c|c|c|c|c|c|c|c|c|c|c|c|}
\hline $\mathrm{HJD}_{\text {mean }}$ & $N_{V}$ & $b_{b v}$ & $r_{b v}$ & $\alpha$ & $N_{B V}$ & $b_{u b}$ & $r_{u b}$ & $\alpha$ & $N_{U B V}$ & $b_{u b v}$ & $r_{u b v}$ & $\alpha$ \\
\hline 8571.9597 & 22 & 0.075 & 0.526 & $0.1<\alpha<0.2$ & - & - & - & - & - & - & - & - \\
\hline 8654.7577 & 9 & 0.201 & 0.333 & $\alpha>0.2$ & 9 & 0.488 & 0.245 & $\alpha>0.2$ & 8 & -0.343 & -0.228 & $\alpha>0.2$ \\
\hline 8915.4620 & 19 & 0.053 & 0.524 & $\alpha<0.05$ & 19 & -0.024 & 607 & $\alpha<0.05$ & 17 & & & $>0.2$ \\
\hline 8991.1227 & 31 & 0.062 & 0.320 & $0.05<\alpha<0.1$ & 31 & 0.158 & 0.320 & $0.05<\alpha<0.1$ & 31 & -0.366 & -0.179 & $x>0.2$ \\
\hline 9292.9221 & 26 & 0.051 & 0.287 & $0.1<\alpha<0.2$ & 26 & 0.141 & 0.287 & $0.1<\alpha<0.2$ & 26 & 0.393 & 0.173 & $x>0.2$ \\
\hline 9376.0888 & 20 & 0.163 & 0.595 & $\alpha<0.05$ & 20 & 0.113 & 0.595 & $\alpha<0.05$ & 20 & -0.019 & -0.012 & $\alpha>0.2$ \\
\hline 9428.6472 & 9 & 0.084 & 0.711 & $\alpha<0.05$ & 9 & -0.095 & 0.693 & $\alpha<0.05$ & 8 & 0.221 & 0.107 & $\alpha>0.2$ \\
\hline 9714.4868 & 15 & 0.063 & 0.251 & $\alpha>0.2$ & 15 & -0.077 & 0.294 & $\alpha>0.2$ & 14 & 1.294 & 0.460 & $0.1<\alpha<0.2$ \\
\hline 10019.4179 & 40 & 0.095 & 0.225 & $0.05<\alpha<0.1$ & 40 & 0.162 & 0.295 & $\alpha<0.05$ & 26 & -0.343 & -0.267 & $\alpha<0.05$ \\
\hline 068.7104 & 39 & 0.067 & 0.282 & $\alpha<0.05$ & 39 & 0.064 & .419 & $\alpha<0.05$ & 28 & 0.293 & 0.240 & $0.05<\alpha<0.1$ \\
\hline 133.6861 & 32 & 0.137 & 0.473 & $\alpha<0.05$ & 32 & .103 & .473 & $\alpha<0.05$ & 32 & 0.382 & 0.489 & $\alpha<0.05$ \\
\hline 421.4340 & 66 & 0.138 & .711 & $\alpha<0.05$ & 66 & .080 & 34 & $\alpha<0.05$ & 62 & 0.197 & 0.157 & $\alpha<0.05$ \\
\hline 10500.5645 & 38 & 0.141 & .755 & $\alpha<0.05$ & 38 & 0.166 & & $\alpha<0.05$ & 34 & 0.542 & 0.414 & $\alpha<0.05$ \\
\hline 10762.9282 & 47 & 158 & 32 & $\alpha<$ & 47 & 125 & & $\alpha<$ & 43 & 0.628 & 603 & .05 \\
\hline & 44 & .142 & 82 & $\alpha<$ & 44 & 162 & & & 32 & 0.579 & 451 & 0.05 \\
\hline 15 & 21 & 140 & 83 & $\alpha<$ & 21 & & & 05 & 20 & 143 & 360 & $x<$ \\
\hline 96 & 29 & 147 & 658 & $\alpha<$ & 29 & .119 & & $\alpha<$ & 28 & 0.358 & & $0.1<$ \\
\hline & 21 & 188 & 648 & $\alpha<0$ & 21 & .230 & & $\alpha<0$ & 15 & 0.368 & 0.266 & $\alpha>0.2$ \\
\hline 4291 & 46 & 0.084 & 396 & $\alpha<0$ & 46 & .061 & & $\alpha<0.05$ & 43 & -0.172 & -0.156 & $0.1<\alpha<0$. \\
\hline 590 & 28 & 0.106 & 396 & $\alpha<0$ & 28 & 0.121 & 363 & $0.05<\alpha<0.1$ & 27 & -0.042 & -0.035 & $\alpha>0.2$ \\
\hline .8130 & 22 & 0.132 & 0.683 & $\alpha<0.05$ & 22 & 0.134 & 0.674 & $\alpha<0.05$ & 21 & 0.087 & 0.055 & $\alpha>0.2$ \\
\hline .5772 & 57 & 0.086 & 0.512 & $\alpha<0.05$ & 57 & 0.169 & .537 & $\alpha<0.05$ & 52 & 0.651 & .363 & $\alpha<0.05$ \\
\hline .6794 & 18 & 0.120 & 0.456 & $0.1<\alpha<0.2$ & 18 & .260 & 56 & $0.1<\alpha<0$. & 18 & 0.561 & 76 & $\alpha>0.2$ \\
\hline & 21 & 0.149 & 0.376 & $0.1<\alpha<0.2$ & 21 & .281 & 76 & $0.1<\alpha<0$. & 21 & 20 & & \\
\hline & 25 & 0.159 & 0.633 & $\alpha<0.05$ & 25 & & & $\alpha<$ & 25 & 99 & & .05 \\
\hline & 28 & 208 & & $\alpha<0$ & 28 & & & & 26 & 0.846 & & \\
\hline & 43 & 0.1 & 9 & & 43 & & & & 39 & $-0 .($ & & \\
\hline & 51 & 0.201 & 77 & & 51 & & & & 51 & & & \\
\hline & 35 & 0.0 & 0 & $0.05<$ & 35 & & & & 34 & & & $0.1<$ \\
\hline & 115 & & & & 115 & & & & 112 & & & $0.1<$ \\
\hline & 44 & 0 & 0 & $\alpha<$ & 44 & & & & 42 & & & $\alpha<$ \\
\hline & 120 & 0.139 & 0.740 & $\alpha<0$ & 120 & 0.149 & 0.745 & $\alpha<$ & 116 & 0.602 & 53 & \\
\hline \multicolumn{13}{|c|}{ DH Leo } \\
\hline 200 & 21 & -0.154 & 0.264 & $\alpha>0.2$ & 21 & 0.262 & .264 & $\alpha>0.2$ & 21 & .294 & & $\alpha>0.2$ \\
\hline 9777 & 18 & 0.086 & 218 & $\alpha>0.2$ & 18 & 0.236 & & $\alpha>0.2$ & 18 & -0.074 & -0.051 & $\alpha>0.2$ \\
\hline 10501.5779 & 9 & -1.363 & -0.597 & $0.1<\alpha<0.2$ & 9 & 0.276 & -0.597 & $.1<\alpha<0$ & 9 & -0.422 & -0.664 & $0.05<\alpha<0$ \\
\hline 60.3633 & 9 & 0.562 & 0.550 & $0.1<\alpha<0.2$ & 9 & -0.236 & 550 & $0.1<\alpha<0$ & 9 & -0.043 & -0.060 & $\alpha>0.2$ \\
\hline 3282 & 12 & -0.036 & -0.202 & $\alpha>0.2$ & 12 & 0.107 & -0.202 & $\alpha>0.2$ & 12 & -0.292 & -0.122 & $\alpha>0.2$ \\
\hline 519 & 10 & 0.097 & 0.243 & $\alpha>0.2$ & 10 & 212 & 243 & $\alpha>$ & 10 & -0.184 & -0.186 & $\alpha>0.2$ \\
\hline & 8 & 0.077 & .113 & $\alpha>$ & 8 & .076 & & & 8 & 0.037 & & \\
\hline & 6 & -0.094 & -0.192 & $\alpha>0.2$ & 6 & -0.262 & -0.192 & $\alpha>0.2$ & 6 & 0.064 & 0.044 & $\alpha>0.2$ \\
\hline & 10 & 0.252 & 55 & $0.1<\alpha<0.2$ & 10 & 0.385 & 0.555 & $0.1<\alpha<0.2$ & 10 & 1.156 & 0.528 & $0.1<\alpha<0.2$ \\
\hline & 5 & -1.222 & -0.3 & & - & - & - & - & - & - & - & - \\
\hline & 8 & & & & 8 & 0.452 & & & 8 & -0.685 & .334 & \\
\hline & 26 & & & & 26 & & & & 26 & & & \\
\hline & 42 & & -0.090 & $\alpha>0.2$ & 42 & -0.041 & -0.0 & & 42 & & & \\
\hline \multicolumn{13}{|c|}{ HU Vir } \\
\hline & 23 & & & $\alpha<$ & 23 & & & $<$ & 22 & .223 & & \\
\hline & 13 & & & 5 & 13 & & & & 3 & .391 & .430 & \\
\hline & 22 & 120 & & $\alpha<0$ & 22 & & & $<$ & 12 & 1.184 & 0.468 & $0.05<\alpha<0$ \\
\hline 997 & 18 & 134 & 954 & $\alpha<0$ & 18 & 195 & & $<0$ & 17 & 1.339 & 0.775 & $\alpha<0.05$ \\
\hline 931 & 28 & 170 & 780 & $\alpha<0$ & 28 & 0.142 & 796 & $<0.05$ & 20 & 0.534 & 0.449 & $\alpha<0$ \\
\hline 3.4762 & 44 & 087 & 343 & $\alpha<0.05$ & 44 & 605 & & $\alpha<0$ & 11 & 1.825 & .512 & $\alpha<0$ \\
\hline 02.7456 & 42 & 0.052 & 0.175 & $0.1<\alpha<0.2$ & 42 & 270 & 469 & $\alpha<0.05$ & 12 & -0.054 & -0.033 & $\alpha>0.2$ \\
\hline 65.6532 & 53 & 0.093 & 0.404 & $\alpha<0.05$ & 53 & 227 & 090 & $0.1<\alpha<0$. & 19 & 0.579 & 0.227 & $\alpha<0.05$ \\
\hline 867 & 59 & 0.081 & 307 & $\alpha<0$ & 59 & & 371 & $\alpha<0$ & 24 & -0.043 & -0.013 & $\alpha>0.2$ \\
\hline & 95 & 0.082 & 324 & $\alpha<0$ & 95 & 0.060 & 0.301 & $\alpha<0.05$ & 37 & 0.876 & 0.250 & $0.05<\alpha<0$ \\
\hline 37.1486 & 40 & .121 & 6 & $\alpha<0$ & - & - & - & - & - & - & - & - \\
\hline 360.4744 & 67 & 0.125 & 0.32 & $\alpha<0.05$ & 67 & & & $\alpha<0.05$ & 32 & .624 & 201 & $\alpha<0.05$ \\
\hline & 101 & 0.051 & 0.137 & & 101 & & & & 44 & -0.645 & -0.2 & $\alpha>0.2$ \\
\hline & 21 & -0.006 & -0.012 & & 21 & 0.907 & & & 6 & -2.074 & & \\
\hline & 12 & 0.151 & & $\alpha<0$ & 12 & 0.217 & 0.853 & $\alpha<0.05$ & 11 & 0.571 & 0.363 & $\alpha>0.2$ \\
\hline & 10 & -0.036 & -0.1 & & - & - & - & - & - & - & - & - \\
\hline & 62 & & & $\alpha<0$ & 62 & 0.127 & & $\alpha<0.05$ & 43 & 578 & 154 & $\alpha<0.05$ \\
\hline 10202.1810 & 24 & 0.028 & 0.126 & $\alpha>0.2$ & 24 & -0.118 & -0.042 & $\alpha>0.2$ & 14 & 0.444 & 0.239 & $\alpha>0.2$ \\
\hline
\end{tabular}


Table 5. continued.

\begin{tabular}{|c|c|c|c|c|c|c|c|c|c|c|c|c|}
\hline $\mathrm{HJD}_{\text {mean }}$ & $N_{V}$ & $b_{b v}$ & $r_{b v}$ & $\alpha$ & $N_{B V}$ & $b_{u b}$ & $r_{u b}$ & $\alpha$ & $N_{U B V}$ & $b_{u b v}$ & $r_{u b v}$ & $\alpha$ \\
\hline 10236.2116 & 19 & 0.028 & 0.111 & $\alpha>0.2$ & 19 & 0.181 & 0.359 & $\alpha>0.2$ & 5 & 0.198 & 0.048 & $\alpha>0.2$ \\
\hline 10423.4667 & 17 & 0.024 & 0.093 & $>0.2$ & 17 & 0.018 & 0.353 & $\alpha>0.2$ & 9 & -0.008 & -0.012 & $x>0.2$ \\
\hline 131 & 30 & 0.074 & 0.212 & $\alpha>0.2$ & 30 & 0.182 & 0.193 & $\alpha>0.2$ & 22 & 0.246 & 0.109 & $>0.2$ \\
\hline 10525.3309 & 45 & 0.136 & 0.330 & $\alpha<0.05$ & 45 & 0.155 & 0.250 & $\alpha<0.05$ & 33 & -0.049 & -0.0 & $x>0.2$ \\
\hline 10566.6972 & 32 & 0.145 & 0.379 & $\alpha<0.05$ & 32 & 0.014 & 0.133 & $\alpha>0.2$ & 20 & 0.078 & 0.102 & $\alpha>0.2$ \\
\hline 10606.6587 & 20 & 0.116 & 0.655 & $\alpha<0.05$ & 20 & 0.044 & 0.964 & $\alpha<0.05$ & 6 & 0.489 & 0.254 & $\alpha>0.2$ \\
\hline 10789.0346 & 25 & 0.099 & 0.618 & $\alpha<0.05$ & 25 & -0.016 & 0.431 & $\alpha<0.05$ & 11 & -0.764 & -0.552 & $\alpha<0.05$ \\
\hline 10856.3146 & 63 & 0.116 & 0.721 & $\alpha<0.05$ & 63 & 0.126 & 0.710 & $\alpha<0.05$ & 34 & 0.814 & 0.541 & $\alpha<0.05$ \\
\hline 10919.2175 & 40 & 0.106 & 0.772 & $\alpha<0.05$ & 40 & 0.053 & 0.715 & $\alpha<0.05$ & 26 & 0.424 & 0.375 & $\alpha<0.05$ \\
\hline 10965.6732 & 34 & 0.090 & 0.661 & $\alpha<0.05$ & 34 & 0.122 & 0.615 & $\alpha<0.05$ & 19 & 0.452 & 0.315 & $.05<\alpha<0.1$ \\
\hline 11155.4593 & 22 & 0.089 & 0.340 & $0.1<\alpha<0.2$ & 22 & 0.047 & 0.501 & $\alpha<0.05$ & 9 & 0.046 & 0.037 & $\alpha>0.2$ \\
\hline 11199.3651 & 33 & 0.093 & 0.403 & $\alpha<0.05$ & 33 & 0.027 & 588 & $\alpha<0.05$ & 18 & 0.664 & 0.395 & $\alpha<0.05$ \\
\hline 112 & 40 & 0.009 & 047 & & 40 & -0.054 & 0.022 & 0.2 & 27 & 0.038 & & $\alpha>0.2$ \\
\hline 113 & 51 & -0.009 & -0.030 & & 51 & 0.173 & 52 & & 21 & 0.909 & & $\alpha<0.05$ \\
\hline 115 & 15 & 168 & 688 & $<0.05$ & 15 & 0.095 & 0.632 & $\alpha<0$ & 6 & -0.699 & -0.1 & $\alpha>0.2$ \\
\hline 346 & 31 & 130 & 373 & $\alpha<0.05$ & 31 & -0.011 & .450 & $\alpha<0.05$ & 18 & 0.111 & .103 & 0.2 \\
\hline 536 & 17 & .086 & 0.548 & $05<\alpha<0$ & 17 & 0.126 & 0.443 & $.1<\alpha<0.2$ & 7 & -0.206 & -0.088 & 0.2 \\
\hline 384 & 39 & 0.068 & 0.424 & $\alpha<0.05$ & 39 & 0.081 & 0.446 & $\alpha<0.05$ & 22 & 0.315 & 0.144 & 0.2 \\
\hline 852 & 25 & 0.139 & 0.810 & $<0$ & 25 & 0.091 & 0.837 & $\alpha<$ & 13 & 0.526 & 0.432 & $\alpha<0.05$ \\
\hline 9.0089 & 44 & 0.102 & 0.685 & $<0.05$ & 44 & 0.097 & 0.763 & $\alpha<$ & 18 & 0.473 & 0.424 & $\alpha<0.05$ \\
\hline 221 & 50 & 0.111 & 0.748 & $\alpha<0.05$ & 50 & 0.129 & 0.791 & $\alpha<$ & 25 & 0.811 & 0.436 & $\alpha<0.05$ \\
\hline 705 & 33 & 0.114 & 0.744 & $\alpha<0$ & 33 & 0.181 & 0.825 & $\alpha<$ & 16 & 0.943 & 0.614 & $\alpha<0.05$ \\
\hline 060.6745 & 28 & 0.148 & 0.666 & $\alpha<0$ & 28 & -0.030 & 0.883 & $\alpha<$ & 14 & -0.355 & -0.298 & $0.1<\alpha<0.2$ \\
\hline 104 & 76 & 0.101 & 0.686 & 05 & 76 & 0.101 & 0.679 & & 38 & 0.490 & & $\alpha<0.05$ \\
\hline & 68 & 0.102 & 0.69 & $\alpha<0$ & 6 & 0.203 & 0.77 & & 2 & 39 & & .05 \\
\hline & 53 & 0.102 & 0.6 & & 5 & & 0.6 & & 2 & & 46 & .05 \\
\hline & 28 & 0.109 & 0.5 & & 28 & 0.3 & 0.6 & & 4 & 0.700 & 0.262 & $0.1<\alpha<0.2$ \\
\hline & 56 & 0.082 & 0.3 & & 5 & 0.1 & 04 & & 2 & & 27 & \\
\hline & 38 & 0.0 & 3 & & & & 46 & & 2 & 0.379 & 52 & $05<$ \\
\hline & 35 & 0.10 & & & 35 & -0.0 & 0.282 & $05<$ & 11 & -1.0 & & \\
\hline & 50 & 101 & 5 & & 50 & 0.328 & 0.470 & $\alpha<$ & 27 & 0.913 & & .05 \\
\hline & 44 & 031 & 107 & .2 & 44 & 0.329 & 0.136 & $1<c$ & 20 & -0.138 & -0 . & $\alpha>$ \\
\hline 240 & 43 & -0.088 & -0.177 & $\alpha<0.2$ & 43 & 0.231 & -0.295 & $\alpha<$ & 14 & 0.407 & & $\alpha<0.05$ \\
\hline 240 & 43 & 088 & -0.177 & $<\alpha<0.2$ & 43 & 0.231 & -0.295 & 05 & 14 & 0.407 & 0.368 & .05 \\
\hline \multicolumn{13}{|c|}{ RS CVn } \\
\hline & 38 & -0.409 & -0.690 & $\alpha<0$ & 38 & -0.130 & -0.693 & $\alpha<0$ & 23 & 0.129 & & $\alpha>0.2$ \\
\hline & 51 & 179 & 394 & $<0$ & 51 & .101 & & $<$ & 50 & -0.150 & -0.128 & $0.1<\alpha<0$ \\
\hline & 30 & -0.276 & -0 & 5 & 30 & 016 & & & 26 & -0.178 & -0.132 & $\alpha>$ \\
\hline & 70 & -0.256 & -0 & & 70 & -0.376 & & & 66 & 0.146 & 0.086 & $0.1<c$ \\
\hline & 33 & 298 & & & 3 & & & & 60 & & -0 & \\
\hline & 46 & & & 5 & 46 & 112 & & $\alpha<$ & 45 & & -0. & 0.2 \\
\hline & 8 & 186 & 630 & $0.1<c$ & 8 & .321 & 0.630 & $0.1<0$ & 8 & -0.369 & -0.192 & 0.2 \\
\hline & 49 & -0 & & & 49 & & & & 46 & & 66 & .05 \\
\hline & 68 & 278 & & & 68 & & & & 67 & & 75 & $0.1<$ \\
\hline & 113 & .232 & & & 113 & -0.203 & & & 110 & 0.047 & 0.036 & \\
\hline & 47 & -0.179 & -0.753 & & 47 & -0.240 & -0.7 & & 46 & 0.426 & 0.284 & .05 \\
\hline & 66 & -0.224 & -0.601 & & 66 & & -0.606 & & 65 & 0.079 & 0.057 & $<$ \\
\hline 568 & 40 & -0.175 & -0.434 & & 40 & -0.180 & -0.450 & 5 & 37 & 0.205 & 0.187 & $0.1<\alpha<$ \\
\hline & 52 & -0.190 & -0.589 & & 52 & -0.125 & -0.582 & $\alpha<$ & 51 & -0.181 & -0.163 & $0.05<\alpha<0.1$ \\
\hline & 67 & -0.141 & -0 & & 67 & -0.181 & -0.544 & $<$ & 6 & 0.442 & 0.268 & $\alpha<0.05$ \\
\hline & 59 & -0.199 & -0.788 & & 59 & -0.124 & -0.795 & $\alpha<$ & 56 & 0.131 & 0.087 & $0.1<\alpha<0$ \\
\hline & 71 & -0 . & -0.694 & & 71 & -0.263 & -0.685 & $<$ & 8 & 0.441 & 0.291 & $\alpha<0.05$ \\
\hline & 27 & & & & 27 & -0.184 & & & 26 & 0.362 & 0.347 & $0.05<\alpha<0$ \\
\hline 23282.2225 & 54 & -0.221 & -0.747 & $\alpha<0.05$ & 54 & -0.209 & -0.754 & $\alpha<0.05$ & 53 & 0.316 & 0.253 & $\alpha<0.05$ \\
\hline \multicolumn{13}{|c|}{ V775 Her } \\
\hline & 6 & -0.092 & -0.284 & & - & - & - & - & - & — & 一 & - \\
\hline & 18 & 0.208 & 0.442 & 5 & - & - & - & - & - & - & - & - \\
\hline & 10 & & 0.782 & $\alpha<0.05$ & - & - & - & - & - & - & - & - \\
\hline & 20 & & 0.568 & $\alpha<0.05$ & 20 & -0.007 & 0.706 & $\alpha<0.05$ & 10 & -0.166 & -0.161 & $\alpha>0.2$ \\
\hline & 17 & 0.145 & 0.385 & $0.1<\alpha<0.2$ & - & - & - & - & - & - & - & - \\
\hline & 24 & 0.028 & 0.1 & & 24 & 0.049 & -0.045 & $\alpha>0.2$ & 7 & & & $\alpha<0.05$ \\
\hline & 19 & 0.077 & & & 19 & -0.370 & 0.464 & $0.05<\alpha<0$ & & -0.363 & -0.348 & $\alpha>0.2$ \\
\hline & 15 & & & & 15 & -0.381 & 0.035 & $\alpha>0.2$ & 7 & 0.264 & 0.113 & $\alpha>0.2$ \\
\hline & 9 & -0.235 & -0.539 & $0.05<\alpha<0.1$ & - & - & - & - & - & - & - & - \\
\hline 9161.8643 & 27 & 0.086 & 0.374 & $\alpha>0.2$ & - & - & - & - & - & - & - & - \\
\hline
\end{tabular}


Table 5. continued.

\begin{tabular}{|c|c|c|c|c|c|c|c|c|c|c|c|}
\hline $\mathrm{HJD}_{\text {mean }} N_{V}$ & $b_{b v}$ & $r_{b v}$ & $\alpha$ & $N_{B V}$ & $b_{u b}$ & $r_{u b}$ & $\alpha$ & $N_{U B V}$ & $b_{u b v}$ & $r_{u b v}$ & $\alpha$ \\
\hline $9360.5367 \quad 18$ & 0.292 & 0.669 & $\alpha<0.05$ & 18 & 0.212 & 0.808 & $\alpha<0.05$ & 12 & 0.780 & 0.501 & $0.05<\alpha<0.1$ \\
\hline 9534.565922 & 0.168 & 0.177 & $\alpha>0.2$ & 22 & 0.219 & 0.016 & $\alpha>0.2$ & 12 & 0.358 & 0.210 & $\alpha>0.2$ \\
\hline 9881.719827 & 0.131 & 0.314 & $0.1<\alpha<0.2$ & 27 & -0.373 & 0.117 & $\alpha>0.2$ & 16 & -1.067 & -0.392 & $0.05<\alpha<0.1$ \\
\hline 10006.597824 & 0.278 & 0.430 & $0.05<\alpha<0.1$ & 24 & 0.110 & 0.372 & $0.1<\alpha<0.2$ & 11 & 0.610 & 0.259 & $\alpha>0.2$ \\
\hline 257.701920 & -0.061 & -0.081 & $\alpha>0.2$ & 20 & 0.692 & -0.230 & $\alpha>0.2$ & 15 & -0.663 & -0.491 & $0.05<\alpha<0.1$ \\
\hline $12.4971 \quad 18$ & 0.075 & 0.220 & $\alpha>0.2$ & 18 & 0.549 & 0.501 & $0.05<\alpha<0.1$ & 10 & 1.309 & 0.454 & $0.1<\alpha<0.2$ \\
\hline 56.429133 & 0.260 & 0.350 & $\alpha<0.05$ & 33 & 0.199 & 0.605 & $\alpha<0.05$ & 15 & -0.148 & -0.097 & $\alpha>0.2$ \\
\hline $\begin{array}{ll}10599.8688 & 15\end{array}$ & 0.168 & 0.269 & $\alpha>0.2$ & 15 & 0.331 & 0.814 & $\alpha<0.05$ & 9 & 0.360 & 0.139 & $\alpha>0.2$ \\
\hline 10620.376431 & 0.025 & 0.087 & $\alpha>0.2$ & 31 & -0.116 & -0.082 & $\alpha>0.2$ & 15 & 0.048 & 0.025 & $\alpha>0.2$ \\
\hline 10667.006314 & -0.207 & -0.360 & $\alpha>0.2$ & 14 & 0.115 & -0.374 & $\alpha>0.2$ & 12 & -0.095 & -0.069 & $\alpha>0.2$ \\
\hline 10726.466122 & 0.001 & 0.002 & $\alpha>0.2$ & 22 & -0.062 & 0.136 & $\alpha>0.2$ & 15 & 1.633 & 0.447 & $0.05<\alpha<0.1$ \\
\hline 10766.590311 & 0.242 & 0.516 & $0.1<\alpha<0.2$ & 11 & 0.208 & 0.483 & $\alpha>0.2$ & 9 & -0.728 & -0.503 & $0.1<\alpha<0.2$ \\
\hline 10877.498915 & 0.172 & 0.180 & $\alpha>0.2$ & 15 & 1.281 & 0.275 & $\alpha>0.2$ & 8 & 0.639 & 0.150 & $\alpha>0.2$ \\
\hline 10914.902519 & 0.027 & 0.124 & $\alpha>0.2$ & 19 & 0.446 & -0.045 & $\alpha>0.2$ & 9 & 0.596 & 0.160 & $\alpha>0.2$ \\
\hline 5813 & 0.130 & 0.425 & $0.1<\alpha<0.2$ & - & - & - & - & - & - & - & - \\
\hline 13730 & 0.028 & 0.068 & $\alpha>0.2$ & 30 & -0.033 & -0.023 & $\alpha>0.2$ & 19 & 0.222 & 0.150 & $\alpha>0.2$ \\
\hline 11129.102220 & 0.232 & 0.728 & $\alpha<0.05$ & 20 & 0.156 & 0.735 & $\alpha<0.05$ & 13 & 0.694 & 0.342 & $\alpha>0.2$ \\
\hline $11237.0388 \quad 6$ & 0.106 & 0.435 & $0.1<\alpha<0.2$ & 一 & - & - & - & - & - & 一 & - \\
\hline 11277.411521 & 0.125 & 0.347 & $0.1<\alpha<0.2$ & 21 & 0.460 & 0.672 & $\alpha<0.05$ & 14 & 0.736 & 0.179 & $\alpha>0.2$ \\
\hline 11316.372423 & 0.115 & 0.324 & $0.1<\alpha<0.2$ & 23 & 0.354 & 0.141 & $\alpha>0.2$ & 12 & 0.045 & 0.016 & $\alpha>0.2$ \\
\hline $11348.8967 \quad 18$ & 0.100 & 0.341 & $\alpha>0.2$ & 18 & 0.124 & 0.166 & $\alpha>0.2$ & 12 & -0.401 & -0.193 & $\alpha>0.2$ \\
\hline 11461.146826 & 0.198 & 0.394 & $0.05<\alpha<0.1$ & 26 & 0.403 & 0.488 & $\alpha<0.05$ & 18 & 0.524 & 0.309 & $0.1<\alpha<0.2$ \\
\hline 11491.110324 & 0.294 & 0.501 & $\alpha<0.05$ & 24 & 0.479 & 0.213 & $\alpha>0.2$ & 11 & 0.344 & 0.100 & $\alpha>0.2$ \\
\hline 11613.482312 & 0.096 & 0.566 & $0.05<\alpha<0.1$ & 12 & 0.184 & 0.629 & $0.05<\alpha<0.1$ & 9 & -0.686 & -0.237 & $\alpha>0.2$ \\
\hline 11647.904327 & 0.132 & 0.705 & $\alpha<0.05$ & 27 & -0.009 & 0.637 & $\alpha<0.05$ & 17 & -0.035 & -0.012 & $\alpha>0.2$ \\
\hline $684.3448 \quad 18$ & 0.125 & 0.409 & $0.1<\alpha<0.2$ & 18 & 0.086 & 0.629 & $\alpha<0.05$ & 10 & 0.169 & 0.078 & $\alpha>0.2$ \\
\hline 320 & 0.011 & 0.019 & $\alpha>0.2$ & 20 & 0.309 & -0.0 & $\alpha>0.2$ & 16 & 0.289 & 0.172 & $\alpha>0.2$ \\
\hline 025 & 0.188 & 0.767 & $\alpha<0.05$ & 25 & 0.338 & & $\alpha<0.05$ & 11 & 1.229 & 0.590 & $\alpha<0.05$ \\
\hline 319 & 0.214 & 0.553 & $\alpha<0$ & 19 & -0.579 & 0.929 & $\alpha<0.05$ & 9 & -1.652 & -0.490 & $0.05<\alpha<0.1$ \\
\hline 840 & 0.114 & 4 & $\alpha<$ & 40 & 0.543 & & $\alpha<0.05$ & 20 & -0.005 & -0.002 & 0.2 \\
\hline 020 & -0.099 & -0.2 & & 20 & 0.190 & 0.314 & $>0.2$ & 16 & -0.183 & 0.066 & 0.2 \\
\hline 913 & 265 & & $\alpha<0.05$ & 13 & 0.083 & 0.612 & $<\alpha<0$ & 6 & -0.294 & -0.188 & $\alpha>0.2$ \\
\hline 915 & 0.041 & 123 & $\alpha>0.2$ & 15 & -0.038 & 0.537 & $<\alpha<$ & 10 & -2.000 & -0.654 & $\alpha<0.05$ \\
\hline 10610 & 0.144 & 531 & $0.1<\alpha<0.2$ & 10 & 0.100 & 0.505 & $0.1<\alpha<0$ & 8 & 0.455 & 0.330 & $\alpha>0.2$ \\
\hline 12427.385838 & 0.200 & 0.274 & $0.05<\alpha<0.1$ & 38 & 0.083 & 0.275 & $0.05<\alpha<0.1$ & 31 & -0.087 & -0.039 & $\alpha>0.2$ \\
\hline 12570.651021 & 0.064 & 0.286 & $\alpha>0.2$ & 21 & -0.010 & 0.527 & $\alpha<0.05$ & 12 & 0.049 & 0.017 & $\alpha>0.2$ \\
\hline 12714.982015 & 0.026 & 0.100 & $\alpha>0.2$ & 15 & -0.157 & 0.102 & $\alpha>0.2$ & 12 & 1.314 & 0.460 & $0.1<\alpha<0.2$ \\
\hline 65411 & 165 & 767 & $\alpha<0.05$ & - & - & - & - & - & - & - & - \\
\hline 0020 & 115 & 276 & $\alpha>0.2$ & 20 & 373 & 642 & $\alpha<0.05$ & 12 & 0.350 & 0.075 & $\alpha>0.2$ \\
\hline 520 & 082 & 203 & $\alpha>$ & 20 & 0.080 & 221 & $\alpha>0.2$ & 12 & 1.063 & 0.497 & $0.05<\alpha<0.1$ \\
\hline 110 & 062 & & $\alpha>0.2$ & 10 & .062 & 495 & $\alpha>0.2$ & 5 & 0.300 & 0.165 & $\alpha>0.2$ \\
\hline 918 & 072 & & $\alpha>$ & 18 & 011 & 11 & $\alpha>0.2$ & 11 & 1.041 & 0.548 & $\alpha<0.05$ \\
\hline 112 & 0.004 & 009 & $\alpha>$ & 12 & 0.924 & -0.112 & $\alpha>0.2$ & 7 & -1.197 & -0.523 & $0.1<\alpha<0.2$ \\
\hline 13341.850729 & 0.360 & 0.794 & $\alpha<0.05$ & 29 & 0.512 & 0.794 & $\alpha<0.05$ & 12 & 0.466 & 0.122 & $\alpha>0.2$ \\
\hline \multicolumn{12}{|c|}{ AR Lac } \\
\hline $\begin{array}{lll}17982.7438 & 7\end{array}$ & 064 & 052 & .2 & 一 & & - & - & - & 一 & - & - \\
\hline 2716 & 0.053 & 103 & & 16 & 168 & 0.143 & $\alpha>0.2$ & 15 & -0.711 & -0.407 & $\alpha>$ \\
\hline 1921 & -0.051 & -0.143 & & 21 & & & $<0.05$ & 6 & -2.258 & -0.732 & $\alpha<$ \\
\hline 6.789255 & -0.126 & -0.232 & $\alpha<0$ & 55 & -0.330 & -0.288 & $<0.05$ & 51 & 0.472 & 0.330 & $\alpha<0.05$ \\
\hline 7.569065 & -0.274 & -0.361 & $\alpha<0.05$ & 65 & 0.061 & -0.463 & $\alpha<0.05$ & 60 & -0.433 & -0.358 & $\alpha<0.05$ \\
\hline 19590.158929 & -0.581 & -0.669 & $\alpha<0.05$ & 29 & 0.021 & -0.625 & $\alpha<0.05$ & 27 & -0.239 & -0.361 & $0.05<\alpha<0.1$ \\
\hline 4.631235 & -0.472 & -0.727 & $\alpha<0.05$ & 35 & -0.264 & -0.730 & $\alpha<0.05$ & 34 & 0.382 & 0.520 & $\alpha<0.05$ \\
\hline 20036.196638 & 0.018 & 0.052 & $\alpha>0.2$ & 38 & -0.205 & -0.065 & $\alpha>0.2$ & 29 & 0.131 & 0.069 & $\alpha>0.2$ \\
\hline 4.024034 & -0.544 & -0.625 & $\alpha<0.05$ & 34 & 0.276 & -0.618 & $\alpha<0.05$ & 32 & -0.605 & -0.646 & $\alpha<0.05$ \\
\hline 5.425836 & -0.249 & -0.414 & $\alpha<0.05$ & 36 & -0.076 & -0.386 & $\alpha<0.05$ & 33 & 0.739 & 0.494 & $\alpha<0.05$ \\
\hline $3.0217 \quad 15$ & -0.213 & -0.164 & $\alpha>0.2$ & - & - & - & - & - & - & - & - \\
\hline 20755.791034 & 0.064 & 0.087 & $\alpha>0.2$ & 34 & 0.046 & 0.140 & $\alpha>0.2$ & 31 & -0.069 & -0.029 & $\alpha>0.2$ \\
\hline 20961.442922 & 0.021 & 0.088 & $\alpha>0.2$ & 22 & -0.495 & 0.081 & $\alpha>0.2$ & 21 & 1.210 & 0.346 & $0.1<\alpha<0.2$ \\
\hline 21133.190130 & -0.003 & -0.006 & $\alpha>0.2$ & 30 & 0.098 & -0.023 & $\alpha>0.2$ & 28 & -0.933 & -0.438 & $\alpha<0.05$ \\
\hline 21342.262927 & -0.363 & -0.318 & $0.1<\alpha<0.2$ & 27 & 0.080 & -0.409 & $\alpha<0.05$ & 26 & -0.440 & -0.418 & $\alpha<0.05$ \\
\hline 21476.101624 & -0.128 & -0.201 & & 24 & 0.503 & -0.263 & $\alpha>0.2$ & 22 & -0.150 & -0.111 & $\alpha>0.2$ \\
\hline $21709.3057 \quad 10$ & -0.110 & -0.119 & $\alpha>c$ & 10 & -0.333 & -0.393 & $\alpha>0.2$ & 6 & 0.271 & 0.097 & \\
\hline 21853.206442 & -0.425 & -0.403 & $\alpha<0.05$ & 42 & 0.544 & -0.390 & $\alpha<0.05$ & 40 & -1.117 & -0.751 & $\alpha<0.05$ \\
\hline 22151.783239 & -0.059 & -0.101 & $\alpha>0.2$ & 39 & 0.102 & -0.099 & $\alpha>0.2$ & 37 & -0.193 & -0.108 & $\alpha>0.2$ \\
\hline
\end{tabular}


Table 5. continued.

\begin{tabular}{|c|c|c|c|c|c|c|c|c|c|c|c|}
\hline $\mathrm{HJD}_{\text {mean }} N_{V}$ & $b_{b v}$ & $r_{b v}$ & $\alpha$ & $N_{B V}$ & $b_{u b}$ & $r_{u b}$ & $\alpha$ & $N_{U B V}$ & $b_{u b v}$ & $r_{u b v}$ & $\alpha$ \\
\hline 22517.284138 & 0.010 & 0.020 & $\alpha>0.2$ & 38 & 0.104 & 0.020 & $\alpha>0.2$ & 38 & -0.654 & -0.402 & $\alpha<0.05$ \\
\hline $22785.4329 \quad 6$ & -0.274 & -0.461 & $\alpha>0.2$ & 6 & -1.071 & -0.461 & $\alpha>0.2$ & 6 & 1.380 & 0.658 & $0.1<\alpha<0.2$ \\
\hline 22951.228732 & -0.257 & -0.731 & $\alpha<0.05$ & 32 & -0.204 & -0.731 & $\alpha<0.05$ & 32 & 0.421 & 0.384 & $\alpha<0.05$ \\
\hline 23247.281843 & \multicolumn{10}{|c|}{ SZ Psc } & $\alpha>0.2$ \\
\hline $9289.8680 \quad 13$ & -0.059 & -0.179 & $\alpha>0.2$ & 13 & -0.093 & -0.179 & $\alpha>0.2$ & 13 & -0.882 & -0.632 & $\alpha<0.05$ \\
\hline 4319 & -0.237 & -0.543 & $\alpha<0.05$ & 19 & -0.229 & -0.543 & $\alpha<0.05$ & 19 & -0.102 & -0.102 & $\alpha>0.2$ \\
\hline 9974.428614 & -0.031 & -0.081 & $\alpha>0.2$ & 14 & -0.368 & -0.081 & $\alpha>0.2$ & 14 & -0.292 & -0.220 & $\alpha>0.2$ \\
\hline 10278.498910 & -0.662 & -0.813 & $\alpha<0.05$ & 10 & 0.116 & -0.813 & $\alpha<0.05$ & 10 & -0.341 & -0.473 & $\alpha>0.2$ \\
\hline $10385.8886 \quad 4$ & - & - & - & - & - & - & - & - & - & - & - \\
\hline 10753.7941 & -0.278 & -0.797 & $\alpha<0.05$ & 7 & 0.138 & -0.797 & $\alpha<0.05$ & 7 & 0.002 & 0.001 & $\alpha>0.2$ \\
\hline 1.442310 & -0.157 & -0.416 & $\alpha>0.2$ & 10 & -0.349 & -0.416 & $\alpha>0.2$ & 10 & 0.139 & 0.126 & $\alpha>0.2$ \\
\hline $11154.3118 \quad 7$ & -0.598 & -0.541 & $\alpha>0.2$ & 7 & -0.045 & -0.541 & $\alpha>0.2$ & 7 & -0.158 & -0.384 & $\alpha>0.2$ \\
\hline \multicolumn{12}{|c|}{ II Peg } \\
\hline 8638.0881 & 0.189 & 0.850 & $\alpha<0.05$ & - & - & - & - & - & - & - & - \\
\hline 8806.4334 & 0.400 & 0.748 & $\alpha<0.05$ & - & - & - & - & - & - & - & - \\
\hline 8889.449920 & 0.190 & 0.760 & $\alpha<0.05$ & 20 & 0.186 & 0.760 & $\alpha<0.05$ & 20 & 0.389 & 0.382 & $0.1<\alpha<0.2$ \\
\hline 8951.062016 & 0.089 & 0.568 & $\alpha<0.05$ & 16 & 0.169 & 0.725 & $\alpha<0.05$ & 13 & 1.003 & 0.577 & $\alpha<0.05$ \\
\hline $9178.4441 \quad 7$ & 0.031 & 0.258 & $\alpha>0.2$ & 7 & -0.169 & -0.034 & $\alpha>0.2$ & 5 & -1.000 & -0.361 & $\alpha>0.2$ \\
\hline 9255.173431 & 0.112 & 0.591 & $\alpha<0.05$ & 31 & 0.195 & 0.525 & $\alpha<0.05$ & 27 & 0.309 & 0.209 & $\alpha>0.2$ \\
\hline $9329.6697 \quad 10$ & 0.066 & 0.485 & $\alpha>0.2$ & 10 & 0.101 & 0.397 & $\alpha>0.2$ & 6 & -0.166 & -0.092 & $\alpha>0.2$ \\
\hline 9564.065415 & 0.130 & 0.926 & $\alpha<0.05$ & 15 & 0.036 & 0.945 & $\alpha<0.05$ & 13 & 0.203 & 0.309 & $\alpha>0.2$ \\
\hline 9633.997217 & 0.103 & 0.532 & $05<\alpha<0.1$ & 17 & 0.090 & 0.519 & $.05<\alpha<0.1$ & 16 & 0.442 & 0.366 & $\alpha>0.2$ \\
\hline $9695.8458 \quad 14$ & 0.106 & 0.668 & $\alpha<0.05$ & 14 & 0.165 & 0.705 & $\alpha<0.05$ & 13 & 0.701 & 0.441 & $0.1<\alpha<0.2$ \\
\hline 9895.262418 & 0.013 & 0.129 & $\alpha>0.2$ & 18 & 0.028 & 0.041 & $\alpha>0.2$ & 14 & 0.049 & 0.042 & $\alpha>0.2$ \\
\hline 9990.138634 & 0.065 & 0.377 & $\alpha<0.05$ & 34 & -0.074 & 0.312 & $05<\alpha<0.1$ & 28 & -0.490 & -0.334 & $\alpha<0.05$ \\
\hline 52.158738 & -0.007 & -0.043 & $\alpha>0.2$ & 38 & -0.028 & -0.073 & $\alpha>0.2$ & 24 & 0.083 & 0.042 & $\alpha>0.2$ \\
\hline 263.536816 & 0.155 & 0.524 & $05<\alpha<0$ & 16 & 0.122 & 01 & $05<\alpha<0$ & 15 & -0.133 & -0.123 & $\alpha>0.2$ \\
\hline 10294.52939 & 0.341 & 0.452 & $\alpha>0.2$ & 9 & 0.165 & 452 & $\alpha>0.2$ & 9 & -0.575 & -0.604 & $0.1<\alpha<0.2$ \\
\hline 7.492211 & 0.134 & 0.494 & $.1<\alpha<0.2$ & 11 & 0.092 & 0.494 & $0.1<\alpha<0.2$ & 11 & -0.517 & -0.465 & $\alpha>0.2$ \\
\hline 7220 & 0.148 & 0.571 & $\alpha<\mathrm{c}$ & 20 & 0.156 & 0.520 & $\alpha<0.05$ & 18 & 0.348 & 0.432 & $0.1<\alpha<0.2$ \\
\hline 40225 & 0.137 & 0.772 & $\alpha<0.05$ & 25 & 0.161 & 77 & $\alpha<0.05$ & 24 & 38 & 0.453 & $\alpha<0.05$ \\
\hline 18114 & 0.076 & 0.487 & $.1<\alpha<0$ & 14 & 0.008 & 0.487 & $0.1<\alpha<0$ & 11 & -0.159 & -0.110 & $>0.2$ \\
\hline 10469.586610 & 0.143 & 0.881 & $<0.05$ & 10 & 0.225 & 0.850 & $\alpha<0.05$ & 8 & 1.021 & 0.595 & $0.1<\alpha<0.2$ \\
\hline 5.915532 & 0.141 & 0.800 & $\alpha<0.05$ & 32 & 0.241 & 0.806 & $\alpha<0.0$ & 29 & 1.163 & 0.572 & $\alpha<0.05$ \\
\hline 10673.992237 & 0.063 & 0.335 & $\alpha<0.05$ & 37 & 0.248 & 0.335 & $\alpha<0.05$ & 37 & 0.726 & 0.225 & $0.1<\alpha<0.2$ \\
\hline 10726.109626 & 0.172 & 0.741 & $\alpha<0.05$ & 26 & 0.126 & 0.741 & $\alpha<0.05$ & 26 & 0.310 & 0.444 & $\alpha<0.05$ \\
\hline 10772.894937 & 0.157 & 0.702 & $\alpha<0.05$ & 37 & 0.108 & 0.702 & $\alpha<0.05$ & 37 & 0.359 & 0.377 & $\alpha<0.05$ \\
\hline $10828.5922 \quad 8$ & 0.194 & 0.369 & $\alpha>0.2$ & 8 & 0.175 & 0.369 & $\alpha>0.2$ & 8 & 0.194 & 0.517 & $\alpha>0.2$ \\
\hline 10981.931322 & 0.223 & & $\alpha<0$ & 22 & 0.080 & & $\alpha<0$ & 22 & 0.219 & 0.441 & $0.05<\alpha<0$. \\
\hline 148 & 0.100 & & $\alpha<0$ & 48 & 0.133 & & $\alpha<0.05$ & 42 & 0.787 & 0.435 & $\alpha<0.05$ \\
\hline 7950 & 0.104 & & $\alpha<0.05$ & 50 & 0.137 & & $\alpha<0.05$ & 47 & 0.875 & 0.526 & $\alpha<0.05$ \\
\hline 5620 & -0.035 & -0.157 & & 20 & 0.055 & -0.098 & $\alpha>0.2$ & 15 & -0.544 & -0.364 & $0.1<\alpha<0.2$ \\
\hline 6743 & & & & 43 & 0.097 & & .05 & 40 & 316 & 521 & $\alpha<0.05$ \\
\hline 853 & & & & 53 & & & $\alpha<($ & 47 & 39 & & $\alpha<0.05$ \\
\hline 4414 & 0.125 & & & 14 & 0.095 & & $\alpha<0.05$ & 11 & 296 & & 0.2 \\
\hline 419 & 0.089 & & $\alpha$ & 19 & 0.258 & 0.465 & $0.05<\alpha<$ & 17 & 0.118 & & 0.2 \\
\hline 11869.545979 & 0.083 & 361 & $\alpha<0.05$ & 79 & 0.118 & 0.862 & $\alpha<0.05$ & 67 & 1.083 & 0.640 & $\alpha<0.05$ \\
\hline 12067.459011 & -0.025 & -0.201 & $>0.2$ & 11 & 0.211 & -0.254 & $\alpha>0.2$ & 8 & -0.598 & -0.236 & $\alpha>0.2$ \\
\hline 12191.623448 & 106 & 573 & $\alpha<0.05$ & 48 & 0.237 & 0.526 & $\alpha<0.05$ & 43 & 1.117 & 0.454 & $\alpha<0.05$ \\
\hline 12264.680437 & 0.174 & 634 & $<0.05$ & 37 & 0.212 & 0.631 & $\alpha<0.05$ & 33 & 0.900 & 0.553 & $\alpha<0.05$ \\
\hline 12474.767723 & 0.108 & 526 & $\alpha<0.05$ & 23 & 0.112 & 0.424 & $0.05<\alpha<0$ & 18 & 1.304 & 0.496 & $\alpha<0.05$ \\
\hline 12604.725276 & 0.085 & 0.664 & $\alpha<0.05$ & 76 & 0.060 & 0.654 & $\alpha<0.05$ & 71 & 0.575 & 0.281 & $\alpha<0.05$ \\
\hline 216 & 0.080 & 521 & $0.05<\alpha<0$. & 16 & 0.080 & & $\alpha<0$ & 13 & 0.225 & 0.178 & $\alpha>0.2$ \\
\hline 12873.896029 & 0.069 & 0.559 & $\alpha<0.05$ & 29 & 0.116 & 0.563 & $\alpha<0.05$ & 28 & 1.037 & 0.443 & $\alpha<0.05$ \\
\hline 12951.746639 & 0.022 & 0.1 & $\alpha>0.2$ & 39 & 0.121 & 0.199 & $0.1<\alpha<0.2$ & 35 & 0.437 & 0.243 & $0.05<\alpha<0.1$ \\
\hline 13006.121621 & 0.092 & 0.3 & $\alpha>0.2$ & 21 & -0.037 & 0.407 & $0.1<\alpha<0.2$ & 19 & 1.941 & 0.472 & $0.05<\alpha<0.1$ \\
\hline $13170.9253 \quad 16$ & 0.109 & & $\alpha>0.2$ & 16 & 0.190 & 0.497 & $0.1<\alpha<0.2$ & 13 & 0.494 & 0.180 & $\alpha>0.2$ \\
\hline 13296.776653 & 0.155 & 0.727 & $\alpha<0.05$ & 53 & 0.252 & 0.782 & $\alpha<0.05$ & 44 & 1.156 & 0.645 & $\alpha<0.05$ \\
\hline \multicolumn{12}{|c|}{ BY Dra } \\
\hline 8025.961013 & & & & - & - & - & - & & — & 一 & - \\
\hline $8180.1438 \quad 10$ & 0.420 & 0.761 & $\alpha<0$ & - & - & - & - & - & - & - & - \\
\hline $8206.5741 \quad 6$ & 0.148 & 0.193 & 05 & - & - & - & - & - & — & - & - \\
\hline 8366.478411 & 0.103 & 0.198 & $\alpha<0.05$ & - & - & - & - & - & - & - & - \\
\hline 8394.926512 & -0.132 & -0.294 & $\alpha>0.2$ & 12 & -0.563 & 0.412 & $\alpha>0.2$ & 5 & 0.614 & 0.222 & $\alpha>0.2$ \\
\hline
\end{tabular}


Table 5. continued.

\begin{tabular}{|c|c|c|c|c|c|c|c|c|c|c|c|}
\hline $\mathrm{HJD}_{\text {mean }} N_{V}$ & $b_{b v}$ & $r_{b v}$ & $\alpha$ & $N_{B V}$ & $b_{u b}$ & $r_{u b}$ & $\alpha$ & $N_{U B V}$ & $b_{u b v}$ & $r_{u b v}$ & $\alpha$ \\
\hline $8550.1205 \quad 17$ & 0.258 & 0.437 & $\alpha>0.2$ & - & - & - & - & - & & & - \\
\hline 8737.971620 & 0.103 & 0.448 & $05<\alpha<0.1$ & 20 & 0.412 & .922 & $\alpha<0.05$ & 9 & 0.758 & 0.196 & $\alpha>0.2$ \\
\hline $8779.4470 \quad 12$ & 0.168 & 0.503 & $0.1<\alpha<0.2$ & 12 & -0.252 & .462 & $\alpha>0.2$ & 7 & 0.601 & 0.337 & $x>0.2$ \\
\hline $8803.3583 \quad 13$ & 0.001 & 0.003 & $\alpha>0.2$ & - & - & - & - & - & - & - & - \\
\hline 8881.921518 & 0.473 & 0.558 & $\alpha<0.05$ & 18 & 0.366 & 0.558 & $\alpha<0.05$ & 18 & 0.086 & 0.062 & $\alpha>0.2$ \\
\hline 8927.961817 & -0.042 & -0.095 & $\alpha>0.2$ & 17 & -0.239 & 0.038 & $\alpha>0.2$ & 9 & -0.188 & -0.083 & $\alpha>0.2$ \\
\hline $9101.4235 \quad 8$ & 0.168 & 0.508 & $0.05<\alpha<0.1$ & - & - & - & - & 一 & - & - & - \\
\hline 9124.441714 & -0.015 & -0.091 & $\alpha>0.2$ & 14 & 0.147 & 0.363 & $\alpha>0.2$ & 6 & 0.414 & 0.131 & $\alpha>0.2$ \\
\hline 9176.204623 & 0.211 & 0.241 & $\alpha>0.2$ & 23 & -0.072 & 0.293 & $\alpha>0.2$ & 10 & -1.332 & -0.681 & $\alpha<0.05$ \\
\hline $9252.6689 \quad 14$ & 0.173 & 0.164 & $\alpha>0.2$ & 14 & 0.708 & 0.338 & $\alpha>0.2$ & 10 & 1.324 & 0.727 & $\alpha<0.05$ \\
\hline $9480.9194 \quad 9$ & -0.231 & -0.368 & $\alpha>0.2$ & - & - & - & - & - & - & - & - \\
\hline $9524.8022 \quad 13$ & 0.038 & 0.033 & $\alpha>0.2$ & 13 & 0.531 & -0.041 & $\alpha>0.2$ & & -1.035 & -0.552 & $0.05<\alpha<$ \\
\hline 9887.948516 & 0.156 & 0.224 & $\alpha>0.2$ & 16 & 0.289 & 0.182 & $\alpha>0.2$ & 11 & -0.523 & -0.239 & $\alpha>0.2$ \\
\hline $9904.9287 \quad 15$ & -0.062 & -0.070 & $\alpha>0.2$ & 15 & -0.974 & 0.010 & $\alpha>0.2$ & 9 & 1.631 & 0.497 & $0.1<\alpha<0$ \\
\hline 616 & 177 & 59 & $\alpha>0.2$ & 16 & 0.811 & 0.215 & $>0.2$ & 12 & -0.467 & -0.180 & $\alpha>0.2$ \\
\hline 3511 & 0.032 & 0.063 & $\alpha>0.2$ & 11 & -0.700 & 0.455 & $\alpha>0.2$ & 6 & -1.465 & -0.609 & $0.05<\alpha<0$ \\
\hline 3620 & -0.155 & -0.201 & $\alpha>0.2$ & - & - & - & - & - & - & - & - \\
\hline 500.008420 & 096 & 139 & $\alpha>0.2$ & 20 & -0.965 & 179 & $\alpha>0.2$ & 15 & -0.481 & -0.181 & $\alpha>0.2$ \\
\hline $507 \quad 16$ & -0.042 & -0.072 & $\alpha>0.2$ & 16 & 0.218 & 0.348 & $\alpha>0.2$ & 10 & -0.093 & -0.044 & $\alpha>0.2$ \\
\hline 10546.903624 & 0.122 & 0.337 & $0.1<\alpha<0.2$ & 24 & 0.380 & 0.229 & $\alpha>0.2$ & 17 & -0.075 & -0.024 & $\alpha>0.2$ \\
\hline $10663.0347 \quad 17$ & -0.144 & -0.117 & $\alpha>0.2$ & 17 & -0.699 & -0.117 & $\alpha>0.2$ & 17 & 0.271 & 0.092 & $\alpha>0.2$ \\
\hline $10893.9312 \quad 5$ & -0.009 & -0.071 & $\alpha>0.2$ & - & - & - & - & - & - & - & - \\
\hline 11025.464811 & -0.029 & -0.149 & $\alpha>0.2$ & 11 & -0.343 & -0.149 & $\alpha>0.2$ & 11 & 0.868 & 0.208 & $\alpha>0.2$ \\
\hline 11067.303211 & 0.097 & 174 & $\alpha>0.2$ & 11 & -0.279 & 0.174 & $\alpha>0.2$ & 11 & 0.275 & 0.167 & $\alpha>0.2$ \\
\hline $11324.4531 \quad 13$ & 0.089 & 0.061 & $\alpha>0.2$ & 13 & 1.327 & 0.061 & $\alpha>0.2$ & 13 & -1.435 & -0.505 & $0.1<\alpha<0.2$ \\
\hline 11645.87229 & -0.078 & -0.081 & $\alpha>0.2$ & 9 & 0.393 & -0.719 & $\alpha<0.05$ & 5 & -0.485 & -0.271 & $\alpha>0.2$ \\
\hline 910 & -0.062 & -0.072 & $\alpha>0.2$ & 10 & -1.857 & -0.072 & $\alpha>0.2$ & 10 & 2.257 & 0.528 & $0.1<\alpha<0.2$ \\
\hline 3.344315 & -0.022 & -0.033 & $\alpha>0.2$ & 15 & 0.593 & -0.033 & $\alpha>0.2$ & 15 & 0.622 & 0.204 & $\alpha>0.2$ \\
\hline 0.356126 & 0.370 & 0.346 & $0.1<\alpha<0.2$ & 26 & -0.099 & 0.772 & $\alpha<0.05$ & 13 & -0.672 & -0.244 & $\alpha>0.2$ \\
\hline 9.096930 & 0.032 & 75 & & 30 & -0.277 & -0.187 & $\alpha>0.2$ & 14 & 0.269 & 0.112 & $\alpha>0.2$ \\
\hline 9011 & 0.065 & & & 11 & & & $\alpha<$ & 5 & -0.104 & -0.048 & $\alpha>0.2$ \\
\hline .999518 & & & $\alpha<0$ & 18 & -0 & 53 & $\alpha<($ & 18 & 255 & -0.224 & $\alpha>0.2$ \\
\hline $58.4988 \quad 23$ & 0.108 & & & 23 & -0.096 & & & 16 & -1.179 & -0.451 & $0.05<\alpha<0$ \\
\hline 24.982323 & -0.040 & -0.0 & & 23 & 0.712 & -0.140 & & 14 & 351 & 169 & $\alpha>0.2$ \\
\hline 794.773244 & 198 & & $<0$ & 44 & -0.120 & 0.380 & $\alpha<0.05$ & 23 & -0.282 & -0.146 & $0.1<\alpha<0.2$ \\
\hline 927.611428 & 0.152 & & $<0.05$ & 28 & 0.010 & 0.571 & $\alpha<0.05$ & 16 & -0.317 & -0.137 & $\alpha>0.2$ \\
\hline 5717 & -0.038 & -0.048 & & 17 & 0.4 & 372 & $\alpha>$ & 8 & -0.099 & -0.041 & $\alpha>$ \\
\hline $13288.6200 \quad 19$ & -0.376 & -0.238 & $\alpha>0.2$ & 19 & 1.097 & -0.311 & $\alpha>0.2$ & 10 & -0.859 & -0.236 & $\alpha>0.2$ \\
\hline
\end{tabular}


S. Messina: Patterns of color variations in close binary systems. I., Online Material $p 17$

Table 6. Average slopes and related uncertainty of the linear fits to: $B-V$ vs. $V, U-B$ vs. $V$ and $U-B$ vs. $B-V$ relations. Only light curves ( $N)$ whose magnitude and colors were correlated with a high significance level $(\alpha<0.1)$ are considered. The slopes' smallest and largest values are also listed.

\begin{tabular}{|c|c|c|c|c|c|}
\hline \multicolumn{6}{|c|}{ Short-term rotational modulation } \\
\hline Target & & $N$ & Average Slope & Min & $\operatorname{Max}$ \\
\hline \multirow[t]{3}{*}{ AR Psc } & $\overline{\langle B V / V\rangle}$ & 4 & $-0.39 \pm 0.15$ & -0.60 & -0.23 \\
\hline & $\langle U B / V\rangle$ & 4 & $-0.12 \pm 0.41$ & -0.40 & 0.47 \\
\hline & $\langle U B / B V\rangle$ & 7 & $-0.70 \pm 0.31$ & -1.16 & -0.15 \\
\hline \multirow[t]{3}{*}{ VY Ari } & $\langle B V / V\rangle$ & 24 & $0.18 \pm 0.07$ & 0.09 & 0.35 \\
\hline & $\langle U B / V\rangle$ & 24 & $0.23 \pm 0.17$ & -0.22 & 0.69 \\
\hline & $\langle U B / B V\rangle$ & 14 & $0.96 \pm 0.37$ & 0.42 & 1.74 \\
\hline \multirow{3}{*}{ UX Ari } & $\langle B V / V\rangle$ & 30 & $-0.19 \pm 0.08$ & -0.45 & -0.09 \\
\hline & $\langle U B / V\rangle$ & 30 & $-0.29 \pm 0.08$ & -0.61 & -0.14 \\
\hline & $\langle U B / B V\rangle$ & 23 & $1.07 \pm 0.52$ & -0.63 & 1.74 \\
\hline \multirow{3}{*}{ V711 Tau } & $\langle B V / V\rangle$ & 8 & $-0.04 \pm 0.13$ & -0.16 & 0.21 \\
\hline & $\langle U B / V\rangle$ & 10 & $-0.16 \pm 0.13$ & -0.46 & 0.04 \\
\hline & $\langle U B / B V\rangle$ & 7 & $-0.41 \pm 0.68$ & -1.69 & 0.63 \\
\hline \multirow[t]{3}{*}{ EI Eri } & $\langle\mathrm{BV} / \mathrm{V}\rangle$ & 17 & $0.12 \pm 0.22$ & -0.37 & 0.36 \\
\hline & $\langle U B / V\rangle$ & 17 & $0.15 \pm 0.22$ & -0.17 & 0.88 \\
\hline & $\langle U B / B V\rangle$ & 8 & $0.09 \pm 0.95$ & -0.93 & 1.48 \\
\hline \multirow[t]{3}{*}{ V1149 Ori } & $\langle B V / V\rangle$ & 27 & $0.13 \pm 0.04$ & 0.05 & 0.21 \\
\hline & $\langle U B / V\rangle$ & 27 & $0.15 \pm 0.09$ & -0.10 & 0.38 \\
\hline & $\langle U B / B V\rangle$ & 13 & $0.57 \pm 0.44$ & -0.34 & 1.50 \\
\hline \multirow[t]{3}{*}{ DH Leo } & $\langle B V / V\rangle$ & 3 & $-0.18 \pm 1.03$ & -1.36 & 0.56 \\
\hline & $\langle U B / V\rangle$ & 3 & $0.14 \pm 0.33$ & -0.24 & 0.39 \\
\hline & $\langle U B / B V\rangle$ & 2 & $0.36 \pm 1.11$ & -0.42 & 1.15 \\
\hline \multirow[t]{3}{*}{ HU Vir } & $\langle B V / V\rangle$ & 38 & $0.11 \pm 0.03$ & 0.07 & 0.17 \\
\hline & $\langle U B / V\rangle$ & 38 & $0.15 \pm 0.14$ & -0.09 & 0.60 \\
\hline & $\langle U B / B V\rangle$ & 27 & $0.60 \pm 0.55$ & -1.08 & 1.82 \\
\hline \multirow[t]{3}{*}{$\overline{R S C V n}$} & $\langle B V / V\rangle$ & 18 & $-0.23 \pm 0.06$ & -0.41 & -0.14 \\
\hline & $\langle U B / V\rangle$ & 18 & $-0.18 \pm 0.09$ & -0.38 & 0.02 \\
\hline & $\langle U B / B V\rangle$ & 7 & $0.34 \pm 0.24$ & -0.18 & 0.60 \\
\hline \multirow[t]{3}{*}{ V775 Her } & $\overline{\langle B V / V\rangle}$ & 20 & $0.18 \pm 0.14$ & -0.23 & 0.37 \\
\hline & $\langle U B / V\rangle$ & 21 & $0.16 \pm 0.28$ & -0.58 & 0.55 \\
\hline & $\langle U B / B V\rangle$ & 10 & $-0.08 \pm 1.35$ & -2.00 & 1.63 \\
\hline \multirow[t]{3}{*}{ AR Lac } & $\overline{\langle B V / V\rangle}$ & 8 & $-0.37 \pm 0.16$ & -0.58 & -0.13 \\
\hline & $\langle U B / V\rangle$ & 10 & $-0.01 \pm 0.26$ & -0.33 & 0.54 \\
\hline & $\langle U B / B V\rangle$ & 12 & $-0.38 \pm 0.83$ & -2.25 & 0.74 \\
\hline \multirow[t]{3}{*}{ II Peg } & $\langle B V / V\rangle$ & 32 & $0.13 \pm 0.06$ & 0.06 & 0.40 \\
\hline & $\langle U B / V\rangle$ & 30 & $0.14 \pm 0.06$ & -0.07 & 0.25 \\
\hline & $\langle U B / B V\rangle$ & 20 & $0.82 \pm 0.51$ & -0.49 & 1.94 \\
\hline \multirow[t]{3}{*}{ BY Dra } & $\langle B V / V\rangle$ & 10 & $0.21 \pm 0.13$ & 0.10 & 0.47 \\
\hline & $\langle U B / V\rangle$ & 8 & $0.11 \pm 0.23$ & -0.12 & 0.41 \\
\hline & $\langle U B / B V\rangle$ & 5 & $-0.73 \pm 1.16$ & -1.46 & 1.32 \\
\hline \multirow[t]{3}{*}{ SZ Psc } & $\overline{\langle B V / V\rangle}$ & 3 & $-0.39 \pm 0.23$ & -0.66 & -0.24 \\
\hline & $\langle U B / V\rangle$ & 3 & $-0.00 \pm 0.24$ & -0.23 & 0.12 \\
\hline & $\langle U B / B V\rangle$ & 1 & $-0.88 \pm-$ & - & - \\
\hline
\end{tabular}


S. Messina: Patterns of color variations in close binary systems. I., Online Material $p 18$

Table 7. Slopes and related uncertainty of the linear fits to: $B-V$ vs. $V, U-B$ vs. $V$ and $U-B$ vs. $B-V$ relations on the long-term time scale, to the light curve brightest magnitude vs. bluest colors $(\min )$ and faintest magnitude vs. reddest colors $(\max )$. Number $N$ of observations, correlation coefficient $(r)$ and related significance level are also listed.

\begin{tabular}{|c|c|c|c|c|}
\hline \multicolumn{5}{|c|}{ Long-term variation } \\
\hline & $N$ & Slope & $r$ & Significance \\
\hline \multicolumn{5}{|c|}{ AR Psc } \\
\hline$B-V / V$ & 595 & $-0.08 \pm 0.01$ & -0.27 & $\alpha<0.01$ \\
\hline$(B-V / V)_{\min }$ & 34 & $-0.08 \pm 0.05$ & -0.28 & $0.05<\alpha<0.1$ \\
\hline$(B-V / V)_{\max }$ & 34 & $-0.10 \pm 0.05$ & -0.34 & $\alpha<0.01$ \\
\hline$U-B / V$ & 558 & $-0.11 \pm 0.01$ & -0.37 & $\alpha<0.01$ \\
\hline$(U-B / V)_{\min }$ & 33 & $-0.10 \pm 0.04$ & -0.38 & $\alpha<0.01$ \\
\hline$(U-B / V)_{\max }$ & 33 & $-0.13 \pm 0.04$ & -0.56 & $\alpha<0.01$ \\
\hline$B / B-V$ & 558 & $-0.00 \pm 0.04$ & -0.00 & $\alpha<0.01$ \\
\hline \multicolumn{5}{|c|}{ VY Ari } \\
\hline$B-V / V$ & 963 & $0.11 \pm 0.01$ & 0.54 & $\alpha<0.01$ \\
\hline$(B-V / V)_{\min }$ & 39 & $0.03 \pm 0.05$ & 0.12 & $0.05<\alpha<0.2$ \\
\hline$(B-V / V)_{\max }$ & 39 & $0.07 \pm 0.02$ & 0.41 & $\alpha<0.01$ \\
\hline$U-B / V$ & 907 & $0.12 \pm 0.01$ & 0.27 & $0.05<\alpha<0.1$ \\
\hline$(U-B / V)_{\min }$ & 38 & $-0.28 \pm 0.15$ & -0.31 & $\alpha<0.01$ \\
\hline$(U-B / V)_{\max }$ & 38 & $0.07 \pm 0.07$ & 0.18 & $0.2<\alpha<0.1$ \\
\hline$U-B / B-V$ & 907 & $0.74 \pm 0.07$ & 0.34 & $\alpha<0.01$ \\
\hline \multicolumn{5}{|c|}{ UX Ari } \\
\hline$B-V / V$ & 1155 & $-0.16 \pm 0.00$ & -0.82 & $\alpha<0.01$ \\
\hline$(B-V / V)_{\min }$ & 37 & $-0.10 \pm 0.03$ & -0.45 & $\alpha<0.01$ \\
\hline$(B-V / V)_{\max }$ & 37 & $-0.07 \pm 0.02$ & -0.52 & $\alpha<0.01$ \\
\hline$U-B / V$ & 1089 & $-0.29 \pm 0.01$ & -0.84 & $\alpha<0.01$ \\
\hline$(U-B / V)_{\min }$ & 36 & $-0.19 \pm 0.06$ & -0.52 & $\alpha<0.01$ \\
\hline$(U-B / V)_{\max }$ & 36 & $-0.13 \pm 0.05$ & -0.39 & $\alpha<0.01$ \\
\hline$B / B-V$ & 1089 & $1.20 \pm 0.04$ & 0.68 & $\alpha<0.01$ \\
\hline \multicolumn{5}{|c|}{ V711 Tau } \\
\hline$B-V / V$ & 732 & $-0.01 \pm 0.01$ & -0.07 & $\alpha<0.01$ \\
\hline$-V / V)_{\min }$ & 31 & $3 \pm 0.03$ & -0.20 & $0.05<\alpha<0.2$ \\
\hline$(B-V / V)_{\max }$ & 31 & $0.01 \pm 0.04$ & 0.04 & $0.05<\alpha<0.2$ \\
\hline$U-B / V$ & 670 & $-0.04 \pm 0.01$ & -0.12 & $0.05<\alpha<0.2$ \\
\hline$(U-B / V)_{\min }$ & 29 & $-0.02 \pm 0.06$ & -0.08 & $0.05<\alpha<0.2$ \\
\hline$(U-B / V)_{\max }$ & 29 & $0.02 \pm 0.07$ & 0.06 & $0.05<\alpha<0.2$ \\
\hline$U-B / B-V$ & 670 & $0.48 \pm 0.06$ & 0.29 & $\alpha<0.01$ \\
\hline \multicolumn{5}{|c|}{ EI Eri } \\
\hline$B-V / V$ & 931 & $0.16 \pm 0.01$ & 0.40 & $\alpha<0.01$ \\
\hline$(B-V / V)_{\min }$ & 43 & $0 \pm 0.05$ & 0.53 & $\alpha<0.01$ \\
\hline$(B-V / V)_{\max }$ & 43 & $0.19 \pm 0.05$ & 0.53 & $\alpha<0.01$ \\
\hline$U-B / V$ & 828 & $0.20 \pm 0.02$ & 0.27 & $\alpha<0.01$ \\
\hline$(U-B / V)_{\min }$ & 42 & $0.34 \pm 0.10$ & 0.49 & $\alpha<0.01$ \\
\hline$(U-B / V)_{\max }$ & 42 & $0.17 \pm 0.10$ & 0.25 & $\alpha<0.01$ \\
\hline$B / B-V$ & 828 & $0.42 \pm 0.06$ & 0.24 & $\alpha<0.01$ \\
\hline \multicolumn{5}{|c|}{ V1149 Ori } \\
\hline$B-V / V$ & 1225 & $0.12 \pm 0.00$ & 0.70 & $\alpha<0.01$ \\
\hline$(B-V / V)_{\min }$ & 35 & 0.02 & 0.71 & $\alpha<0.01$ \\
\hline$(B-V / V)_{\max }$ & 35 & $0.15 \pm 0.03$ & 0.68 & $\alpha<0.01$ \\
\hline$U-B / V$ & 1115 & $0.00 \pm 0.01$ & 0.00 & $\alpha<0.01$ \\
\hline$-B / V)_{\min }$ & 35 & $-0.03 \pm 0.04$ & -0.11 & $0.05<\alpha<0.2$ \\
\hline$-B / V)_{\max }$ & 35 & $0.00 \pm 0.06$ & 0.00 & $0.05<\alpha<0.2$ \\
\hline & 1115 & $0.07 \pm 0.04$ & 0.05 & $\alpha<0.01$ \\
\hline \multicolumn{5}{|c|}{ DH Leo } \\
\hline$B-$ & 184 & $0.03 \pm 0.04$ & 0.06 & $\alpha<0.01$ \\
\hline$(B-V / V)_{\min }$ & 13 & $-0.06 \pm 0.15$ & -0.11 & $\alpha<0.01$ \\
\hline$(B-V / V)_{\max }$ & 13 & $0.14 \pm 0.11$ & 0.36 & $0.05<\alpha<0.2$ \\
\hline$U-B / V$ & 179 & $0.18 \pm 0.06$ & 0.22 & $0.05<\alpha<0.2$ \\
\hline$(U-B / V)_{\min }$ & 12 & $0.35 \pm 0.42$ & 0.26 & $0.05<\alpha<0.2$ \\
\hline$(U-B / V)_{\max }$ & 12 & $0.09 \pm 0.20$ & 0.14 & $0.05<\alpha<0.2$ \\
\hline$U-B / B-V$ & 179 & $-0.17 \pm 0.12$ & -0.11 & $\alpha<0.01$ \\
\hline
\end{tabular}


S. Messina: Patterns of color variations in close binary systems. I., Online Material $p 19$

Table 7. continued.

\begin{tabular}{|c|c|c|c|c|}
\hline \multicolumn{5}{|c|}{ "Long-term variation } \\
\hline & $N$ & Slope & $r$ & \multirow[t]{2}{*}{ Significance } \\
\hline \multirow{9}{*}{$\begin{array}{l}B-V / V \\
(B-V / V)_{\min } \\
(B-V / V)_{\max } \\
U-B / V \\
(U-B / V)_{\min } \\
(U-B / V)_{\max } \\
U-B / B-V\end{array}$} & \multicolumn{3}{|c|}{ HU Vir } & \\
\hline & 2247 & $0.11 \pm 0.00$ & 0.56 & $\alpha<0.01$ \\
\hline & 52 & $0.12 \pm 0.02$ & 0.69 & $\alpha<0.01$ \\
\hline & 52 & $0.12 \pm 0.01$ & 0.81 & $\alpha<0.01$ \\
\hline & 1127 & $0.16 \pm 0.01$ & 0.44 & $\alpha<0.01$ \\
\hline & 50 & $0.19 \pm 0.04$ & 0.54 & $\alpha<0.01$ \\
\hline & 50 & $0.13 \pm 0.03$ & 0.52 & $\alpha<0.01$ \\
\hline & 1127 & $0.72 \pm 0.05$ & 0.38 & $\alpha<0.01$ \\
\hline & \multicolumn{3}{|c|}{ RS CVn } & \\
\hline$B V / V$ & 1311 & $-0.20 \pm 0.01$ & -0.66 & $\alpha<0.01$ \\
\hline$(B V / V)_{\min }$ & 19 & $-0.00 \pm 0.02$ & -0.03 & $\alpha<0.01$ \\
\hline$(B V / V)_{\max }$ & 19 & $0.05 \pm 0.08$ & 0.14 & $\alpha<0.01$ \\
\hline$U B / V$ & 1222 & $-0.00 \pm 0.00$ & -0.01 & $\alpha<0.01$ \\
\hline$(U B / V)_{\min }$ & 19 & $0.02 \pm 0.04$ & 0.13 & $\alpha<0.01$ \\
\hline$(U B / V)_{\max }$ & 19 & $-0.12 \pm 0.13$ & -0.21 & $0.05<\alpha<0.2$ \\
\hline \multirow[t]{2}{*}{$U B / B V$} & 1222 & $0.03 \pm 0.19$ & 0.00 & $\alpha<0.01$ \\
\hline & \multicolumn{3}{|c|}{ V775 Her } & \\
\hline \multirow{7}{*}{$\begin{array}{l}B-V / V \\
(B-V / V)_{\min } \\
(B-V / V)_{\max } \\
U-B / V \\
(U-B / V)_{\min } \\
(U-B / V)_{\max } \\
U-B / B-V\end{array}$} & 1042 & $0.14 \pm 0.01$ & 0.54 & $\alpha<0.01$ \\
\hline & 54 & $0.14 \pm 0.02$ & 0.78 & $\alpha<0.01$ \\
\hline & 54 & $0.15 \pm 0.02$ & 0.71 & $\alpha<0.01$ \\
\hline & 569 & $0.29 \pm 0.02$ & 0.48 & $\alpha<0.01$ \\
\hline & 45 & $0.33 \pm 0.05$ & 0.71 & $\alpha<0.01$ \\
\hline & 45 & $0.30 \pm 0.06$ & 0.59 & $\alpha<0.01$ \\
\hline & 569 & $0.63 \pm 0.09$ & 0.28 & $\alpha<0.01$ \\
\hline \multicolumn{5}{|c|}{ AR Lac } \\
\hline$B V / V$ & 735 & $-0.14 \pm 0.02$ & -0.25 & $\alpha<0.01$ \\
\hline$V)_{\min }$ & 22 & $0.06 \pm 0.10$ & 0.13 & $\alpha<0.01$ \\
\hline$(B V / V)_{\max }$ & 22 & $0.03 \pm 0.05$ & 0.14 & $\alpha<0.01$ \\
\hline$U B / V$ & 614 & $0.00 \pm 0.01$ & 0.03 & $\alpha<0.01$ \\
\hline$(U B / V)_{\min }$ & 18 & $-0.02 \pm 0.16$ & -0.03 & $\alpha<0.01$ \\
\hline$(U B / V)_{\max }$ & 414 & $0.13 \pm 0.02$ & 0.25 & $\alpha<0.01$ \\
\hline$U B / B V$ & 614 & $0.27 \pm 0.12$ & 0.10 & $\alpha<0.01$ \\
\hline \multicolumn{5}{|c|}{ II Peg } \\
\hline$B-V / V$ & 1191 & $0.09 \pm 0.00$ & 0.51 & $\alpha<0.01$ \\
\hline$(B-V / V)_{\min }$ & 45 & $0.10 \pm 0.04$ & 0.33 & $\alpha<0.01$ \\
\hline$(B-V / V)_{\max }$ & 45 & $0.06 \pm 0.02$ & 0.39 & $\alpha<0.01$ \\
\hline$U-B / V$ & 1046 & $0.12 \pm 0.01$ & 0.43 & $\alpha<0.01$ \\
\hline$(U-B / V)_{\min }$ & 43 & $0.14 \pm 0.05$ & 0.39 & $\alpha<0.01$ \\
\hline$(U-B / V)_{\max }$ & 43 & $0.09 \pm 0.03$ & 0.46 & $\alpha<0.01$ \\
\hline$U-B / B-V$ & 1046 & $0.28 \pm 0.05$ & 0.18 & $\alpha<0.01$ \\
\hline \multicolumn{5}{|c|}{ BY Dra } \\
\hline$B-V / V$ & 693 & $0.06 \pm 0.01$ & 0.27 & $\alpha<$ \\
\hline$(B-V / V)_{\min }$ & 43 & $0.04 \pm 0.02$ & 0.28 & $\alpha<0.01$ \\
\hline$(B-V / V)_{\max }$ & 43 & $0.06 \pm 0.02$ & 0.45 & $\alpha<0.01$ \\
\hline$U-B / V$ & 399 & $0.01 \pm 0.03$ & 0.03 & $\alpha<0.01$ \\
\hline$(U-B / V)_{\min }$ & 33 & $0.04 \pm 0.07$ & 0.12 & $0.05<\alpha<0.2$ \\
\hline$(U-B / V)_{\max }$ & 33 & $-0.01 \pm 0.06$ & -0.02 & $0.05<\alpha<0.2$ \\
\hline$U-B / B-V$ & 399 & $-0.58 \pm 0.12$ & -0.24 & $\alpha<0.01$ \\
\hline \multicolumn{5}{|c|}{ SZ Psc } \\
\hline$B-V / V$ & 86 & $-0.18 \pm 0.05$ & -0.35 & $\alpha<0.01$ \\
\hline$(B-V / V)_{\min }$ & 7 & $-0.20 \pm 0.33$ & -0.26 & $\alpha<0.01$ \\
\hline$(B-V / V)_{\max }$ & 7 & $-0.12 \pm 0.24$ & -0.22 & $\alpha<0.01$ \\
\hline$U-B / V$ & 86 & $-0.18 \pm 0.05$ & -0.40 & $0.05<\alpha<0.2$ \\
\hline$(U-B / V)_{\min }$ & 7 & $0.37 \pm 0.20$ & 0.62 & $0.05<\alpha<0.1$ \\
\hline$(U-B / V)_{\max }$ & 7 & $-0.05 \pm 0.16$ & -0.13 & $0.05<\alpha<0.1$ \\
\hline$U-B / B-V$ & 86 & $-0.70 \pm 0.30$ & -0.25 & $0.05<\alpha<0.1$ \\
\hline
\end{tabular}

